This document was prepared in conjunction with work accomplished under Contract No. DE-AC09-96SR18500 with the U. S. Department of Energy.

\title{
DISCLAIMER
}

This report was prepared as an account of work sponsored by an agency of the United States Government. Neither the United States Government nor any agency thereof, nor any of their employees, nor any of their contractors, subcontractors or their employees, makes any warranty, express or implied, or assumes any legal liability or responsibility for the accuracy, completeness, or any third party's use or the results of such use of any information, apparatus, product, or process disclosed, or represents that its use would not infringe privately owned rights. Reference herein to any specific commercial product, process, or service by trade name, trademark, manufacturer, or otherwise, does not necessarily constitute or imply its endorsement, recommendation, or favoring by the United States Government or any agency thereof or its contractors or subcontractors. The views and opinions of authors expressed herein do not necessarily state or reflect those of the United States Government or any agency thereof. 


\section{DEVELOPMENT OF ALTERNATIVE RHEOLOGICAL MEASUREMENTS FOR DWPF SLURRY SAMPLES (U)}

D. C. Koopman

September 2005

Immobilization Technology Section Savannah River National Laboratory Aiken, SC 29808

Prepared for the U.S. Department of Energy Under Contract Number DEAC09-96SR18500

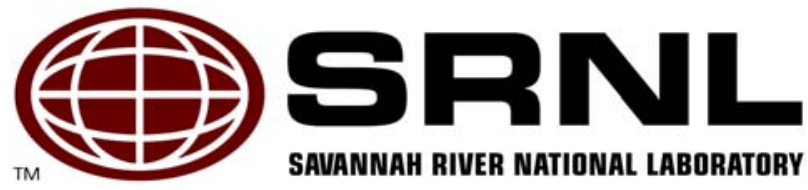




\section{DISCLAIMER}

This report was prepared by Westinghouse Savannah River Company (WSRC) for the United States Department of Energy under Contract No. DE-AC09-96SR18500 and is an account of work performed under that contract. Neither the United States Department of Energy, nor WSRC, nor any of their employees makes any warranty, expressed or implied, or assumes any legal liability or responsibility for the accuracy, completeness, or usefulness, of any information, apparatus, or product or process disclosed herein or represents that its use will not infringe privately owned rights. Reference herein to any specific commercial product, process, or service by trademark, name, manufacturer or otherwise does not necessarily constitute or imply endorsement, recommendation, or favoring of same by WSRC or by the United States Government or any agency thereof. The views and opinions of the authors expressed herein do not necessarily state or reflect those of the United States Government or any agency thereof.

\section{Printed in the United States of America}

Prepared For

U.S. Department of Energy 
Key Words: DWPF, rheology, sludge, SRAT, SME, rheometer, slurry

Retention: permanent

\section{DEVELOPMENT OF ALTERNATIVE RHEOLOGICAL MEASUREMENTS FOR DWPF SLURRY SAMPLES (U)}

D. C. Koopman

September 2005

Immobilization Technology Section Savannah River National Laboratory Aiken, SC 29808

Prepared for the U.S. Department of Energy Under Contract Number DEAC09-96SR18500

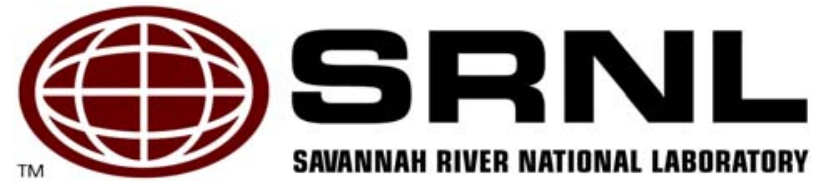




\section{REVIEWS AND APPROVALS}

\section{AUTHOR:}

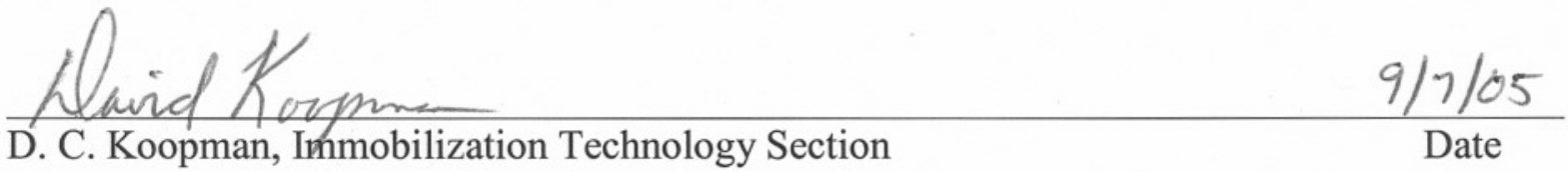

\section{TECHNICAL REVIEWERS:}

E. K. Hansen, Process Development Hanford-RPP Section

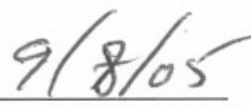

Date

\section{APPROVERS:}

$\begin{array}{lr}\text { Sfonlana for } & 9 / 9 / 05 \\ \text { E. W. Holtzscheiter, Manager, Immobilization Technology Section } & \text { Date } \\ \text { S Inlanc, } & 9 / 9 / 05 \\ \text { S. L. Marra, Manager, Glass Formulation \& Process Development } & \text { Date }\end{array}$




\section{EXECUTIVE SUMMARY}

An experimental study was undertaken to improve existing rheological measurement methods and to develop a suite of supplementary rheological measurements. This study was in parallel with a study examining simulant samples with unusual rheological behavior, Koopman (2005). The rheological data obtained were used to better constrain the choice of free parameters in the traditional controlled shear rate flow curve measurement. One set of constraints was developed for sludges and SRAT products. A second set of constraints was developed for SME products. A set of new rheological measurement methods to supplement the controlled shear rate flow curve method evolved as the testing progressed.

The number of rheological methods applicable to DWPF samples has been increased to include:

- Controlled shear stress flow curve

- Constant shear rate time curve

- Small deformation yield point

- Creep-recovery analysis

- Oscillation stress sweep

- Oscillation frequency sweep

- Oscillation time sweep

The traditional controlled shear rate flow curve measurement gives a reproducible flow curve for a time independent sample and one measure of thixotropy for a time dependent sample. The new measurements listed above provide information beyond that obtained from the controlled shear rate flow curve, i.e. provide more than just data that might be converted into a Bingham plastic yield stress and consistency. The new methods help to clarify the time dependent behavior that was found in some of the simulant slurry samples.

Three primary new methods were developed to supplement the controlled shear rate flow curve. These were the constant shear rate time curve, the controlled shear stress flow curve, and the oscillation stress sweep. The other four new test methods in the list above were examined, but did not appear to be optimal for the available resources and current set of issues.

The leading candidate for full implementation as a supporting test to the controlled shear rate flow curve was the constant shear rate time curve. This test can be performed by all four rheometers currently in service for DWPF slurry samples, both simulant and radioactive. This test gives a clear, quantifiable measure of the time dependence of the rheological properties of a sample.

The controlled shear stress flow curve and the oscillation stress sweep were similar in many respects. Both provide insight into the behavior of slurries at low shear stresses that are not well-characterized by the traditional controlled shear rate flow curve. These new tests both revealed the existence of two yield stress transitions in most of the slurry samples. The low stress transition was a solid-like to liquid-like transition taken to denote the true yield stress of the sample. The high stress transition was marked by a change in behavior associated with a more fully developed flow pattern, e.g. the Bingham plastic yield stress. The oscillation stress sweep also produced information on the elastic and loss moduli, which provide a direct assessment of the solid-like and liquid-like character of the sample. This information was only obtainable through oscillation measurements. These two tests require one of the two advanced rheometers located at Aiken County Technology Laboratory. 
Detailed recommendations for performing the controlled shear rate flow curve, constant shear rate flow time curve, controlled shear stress flow curve, and oscillation stress sweep were formalized and issued as a separate rheology protocol for DWPF slurries, Koopman (2004b). 


\section{TABLE OF CONTENTS}

EXECUTIVE SUMMARY ……………................................................................ ii

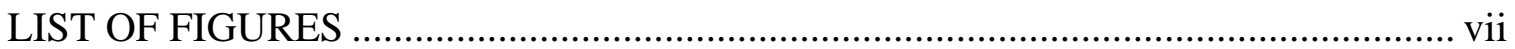

LIST OF TABLES ……………………………….............................................. viii

LIST OF ACRONYMS ......................................................................................... vii

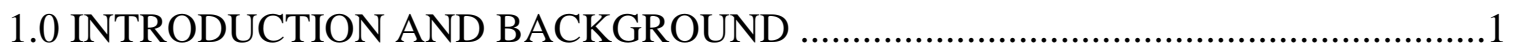

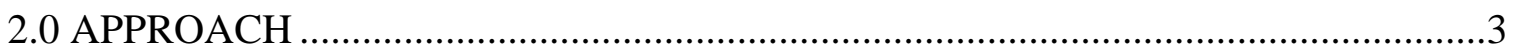

2.1 Simulant Samples ………………………………………………………………..

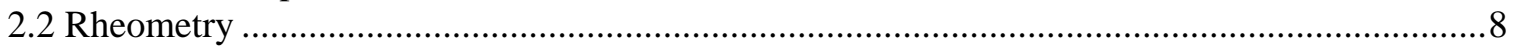

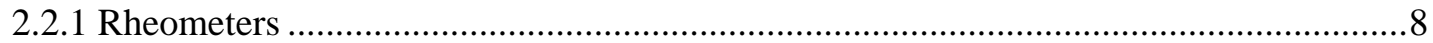

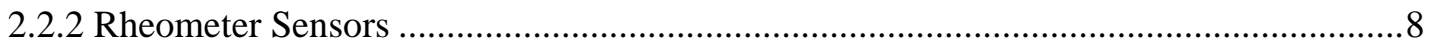

2.2.3 Rheometer Measurements......................................................................................9

2.2.3.1 Controlled Shear Rate Flow Curve.......................................................................9

2.2.3.2 Constant Shear Rate Time Curve .......................................................................

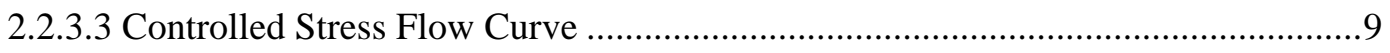

2.2.3.4 Small Deformation Yield Point ............................................................................10

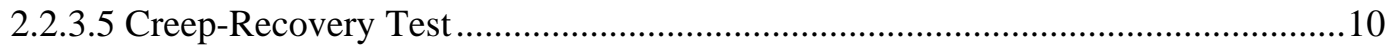

2.2.3.6 Oscillation Frequency Sweep ………………………………………………....11

2.2.3.7 Oscillation Amplitude (Stress) Sweep....................................................................11

2.2.3.8 Oscillation Time Sweep ..................................................................................11

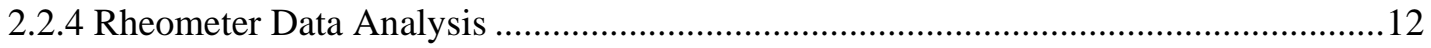

2.2.4.1 Bingham Plastic Fluid Model............................................................................12

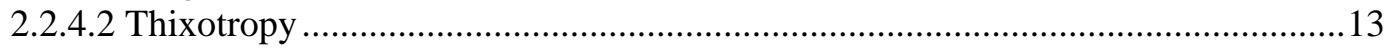

2.2.4.3 Analysis of Controlled Stress Data.......................................................................14

2.2.4.4 Analysis of Small Deformation Yield Point Data ..................................................17

2.2.4.5 Analysis of Creep-Recovery Data ........................................................................17

2.2.4.6 Analysis of Oscillation Frequency Sweep Data ...................................................17

2.2.5 Analysis Matrix ……………………………………………………………....

3.0 RHEOLOGICAL METHOD DEVELOPMENT FINDINGS ………………….........19

3.1 Controlled Shear Rate Flow Curve Studies...........................................................................20

3.1.1 Geometric Effects ...............................................................................................21

3.1.2 Effect of Independent Parameters on the Controlled Shear Rate Flow Curve................24

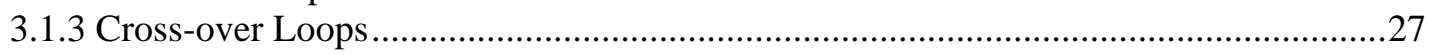

3.1.4 Development of Multi-Curve Measurement..................................................................29

3.1.5 Impact of Taylor Vortices............................................................................................

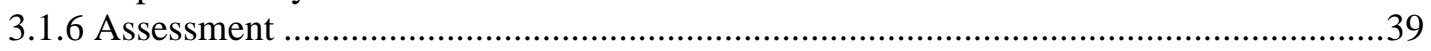

3.2 Controlled Shear Stress Flow Curves..............................................................................42

3.2.1 Controlled Stress Ramp of Viscosity Standard..............................................................43

3.2.2 Controlled Stress Ramps of Process Samples...............................................................44

3.2.3 Assessment ……………………………………………………………………....53

3.3 Constant Shear Rate Time Curves.................................................................................5

3.3.1 Time Curve on Viscosity Standard ............................................................................55

3.3.2 Time Curve on Process Samples .............................................................................56

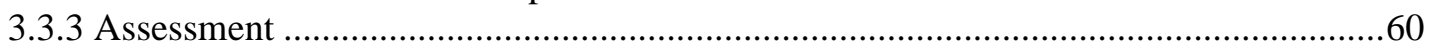

3.4 Other Rotational Measurements ...........................................................................................62

3.4.1 Small Deformation Yield Point Determination .............................................................62

3.4.2 Creep-Recovery Measurements ...............................................................................63

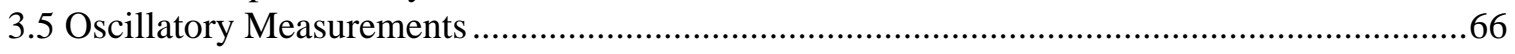


WSRC-TR-2004-00334

Revision 0

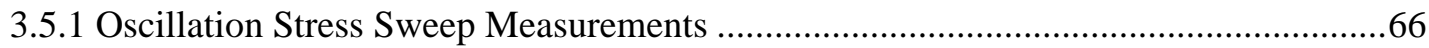

3.5.1.1 Oscillation Stress Sweep on Viscosity Standard ..................................................67

3.5.1.2 Oscillation Stress Sweeps on Process Samples ......................................................67

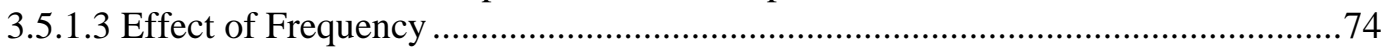

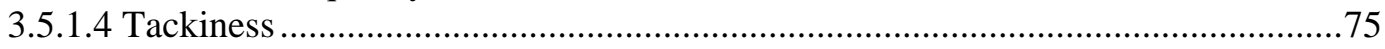

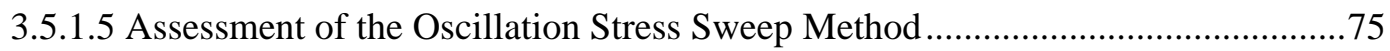

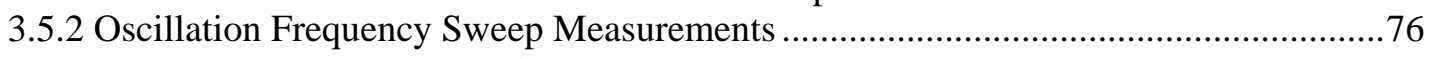

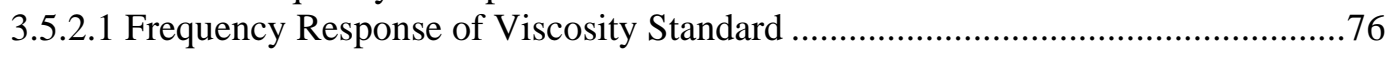

3.5.2.2 Oscillation Frequency Sweeps and Process Samples .............................................77

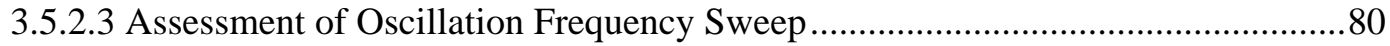

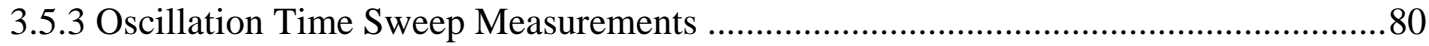

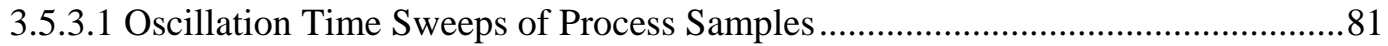

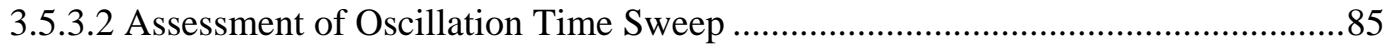

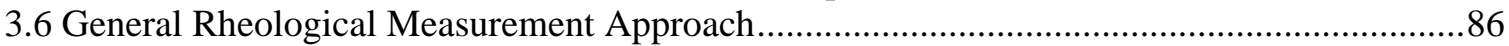

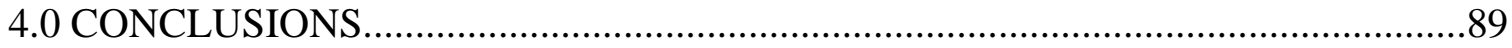

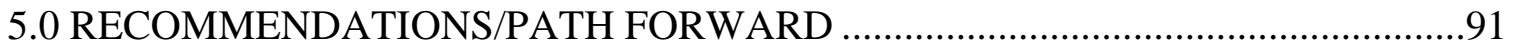

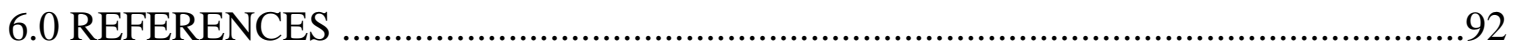

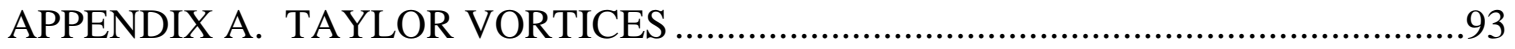




\section{LIST OF FIGURES}

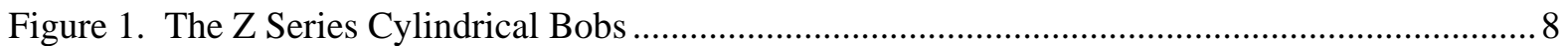

Figure 2. SB2/3 SME Product, Run 5, Heel Study: Effect of Bottom Gap .....................................2 21

Figure 3. SB2/3 CETL SRAT Product by Z41: Effect of Bottom Gap.............................................22

Figure 4. SB2/3 CETL SRAT Product by Z38: Effect of Bottom Gap............................................ 23

Figure 5. Impact of Bob Selection on Flow Curve for SB2 CETL Sludge …...................................23

Figure 6. SB2 CETL SRAT Product: Effect of Five-fold Faster Loop ........................................... 24

Figure 7. Reproducibility of Flow Curves for SB3 CETL SME Product, Z38 …............................. 25

Figure 8. SB3 CETL SME Product: Effect of Faster Loop with Reduced Range, Z38 .................... 26

Figure 9. SB3 CETL SME Product: Reduced Shear Rate Range - Constant Duration, Z38............. 26

Figure 10. Examples of Cross-over Flow Curves for SB2/3 SRAT Product .................................... 27

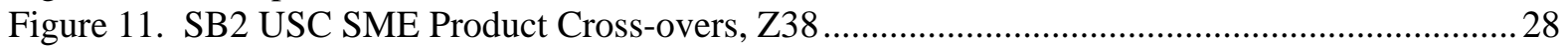

Figure 12. Impact of Dilution on SB2/3 Run 5 SME Product, Z38 ….........................................29

Figure 13. Impact of Consecutive Flow Curve Routine on SB2 CETL Sludge, Z41......................... 30

Figure 14. Consecutive Flow Curves for Diluted SB2/3 CETL SME Product, Z38 ........................... 31

Figure 15. Impact of Consecutive Flow Curves on SB2/3 CETL SME Product, Z38 ....................... 32

Figure 16. Consecutive Flow Curves on 7.5\% DU SRAT Product..............................................33

Figure 17. Impact of Consecutive Flow Curves on 15\% DU Sludge ................................................ 34

Figure 18. Impact of Taylor Vortices on Time-dependence of SB2-3 SME Product ......................... 35

Figure 19. SB2/3 CETL SRAT Product Rheogram and Impact of Taylor Vortices, Z38................... 36

Figure 20. Impact of Taylor Vortices on SB2 CETL Sludge Rheogram.............................................37

Figure 21. SB2/3 SME Product (140\% Stoichiometry), Z38 …....................................................... 38

Figure 22. SB2/3 SME Product (185\% Stoichiometry), Z38 …....................................................... 39

Figure 23. Controlled Stress Ramps of NIST Traceable N35 Viscosity Standard .............................. 43

Figure 24. Controlled Shear Stress Data for SB2 Sludge (USC) .................................................... 44

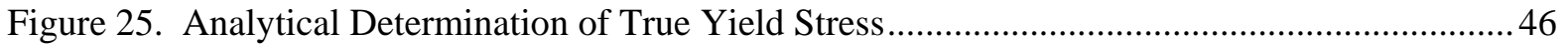

Figure 26. Controlled Stress Compared to Controlled Rate Flow Curve ........................................... 47

Figure 27. Controlled Stress Ramp on Acid Stoichiometry Study SME Products..............................48

Figure 28. Non-typical strain-stress plot of SB2/3 CETL SME product............................................50

Figure 29. SB2 CETL SRAT Product at Two Hold Times, Z41 .......................................................5 52

Figure 30. Constant Shear Rate Time Test of NIST N35 Viscosity Standard ....................................55

Figure 31. Comparison of the Time Dependence of Four Sludge Simulants ...................................... 56

Figure 32. Comparison of the Time Dependence of Three SRAT Products .......................................5 57

Figure 33. Comparison of the Time Dependence of Five SME Products ...........................................5 59

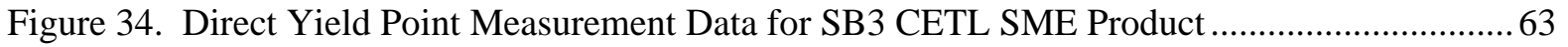

Figure 35. SB2/3 SME Product, Run 6, 155\% Acid - Creep-Recovery Data, Z38 …......................... 64

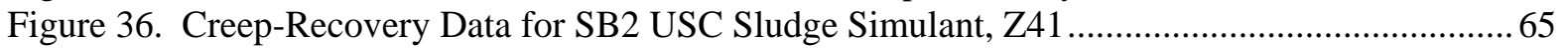

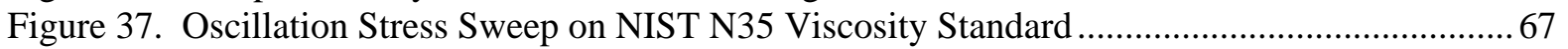

Figure 38. Oscillation Stress Sweep on SB2/3 CETL SME Product (170\% Stoichiometry), Z38 ..... 68

Figure 39. Oscillation Stress Sweep Data for SB2/3 CETL SME Product (170\% Acid), Z38 ...........69

Figure 40. SB2/3 CETL SME Products - Delta Comparison ............................................................ 71

Figure 41. Storage Modulus of Four SB2/3 CETL SME Products ................................................... 72

Figure 42. Oscillation Stress Sweep on SB2/3 CETL SRAT Product ............................................... 73

Figure 43. Effect of Frequency on SB2/3 CETL SME Product Oscillation Stress Sweeps .................74

Figure 44. Example Stress Sweep Data from ThermoHaake ............................................................. 76

Figure 45. NIST N35 Viscosity Standard Response to Oscillation Frequency Sweep ....................... 77

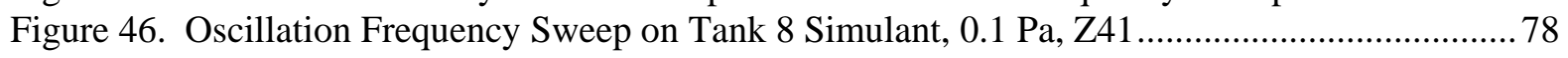

Figure 47. Test of Cox-Moore Rule on Tank 8 Simulant.............................................................. 79 
Figure 48. Oscillation Frequency Sweep on SB2/3 SRAT Product at $0.5 \mathrm{~Pa}$.................................. 80

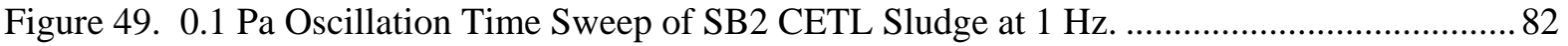

Figure 50. 1.5 Pa Oscillation Time Sweep of SB2 CETL Sludge at $1 \mathrm{~Hz}$....................................... 82

Figure 51. 6 Pa Oscillation Time Sweep of SB2 CETL Sludge at $1 \mathrm{~Hz}$.......................................... 83

Figure 52. $15 \mathrm{~Pa}$ Oscillation Time Sweep of SB2 CETL Sludge at $1 \mathrm{~Hz}$........................................ 83

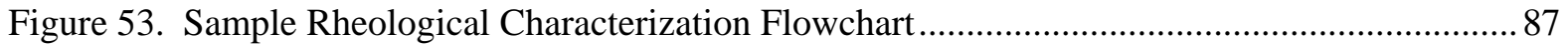

Figure 54. Shear Rate Range Guidance for DWPF Sludge .............................................................. 95

Figure 55. Shear Rate Range Guidance for DWPF SRAT Products ..................................................96

Figure 56. Shear Rate Range Guidance for DWPF SME Products ................................................... 97

\section{LIST OF TABLES}

Table 1. Physical Properties of Rheology Slurry Samples …........................................................

Table 2. Summary of Tests Performed by Sample and Test Type ................................................... 18

Table 3. Summary of Recommended Flow Curve Inputs .................................................................. 41

Table 4. Changes in Wt. \% Total Solids of SB2/3 SME Products ......................................................49

Table 5. Structure of Controlled Shear Stress vs. Controlled Shear Rate Flow Curve ....................... 54

Table 6. Average Apparent Viscosity at 200/s Shear Rate of Four Sludge Simulants........................57

Table 7. Average Apparent Viscosity of Three SRAT Products...................................................... 58

Table 8. Average Apparent Viscosities of SME Products...............................................................59

Table 9. SME Yield Stress Comparison of Controlled and Oscillation Stress Methods..................... 70

Table 10. Yield Stress Data, Pa, for Sludges and SRAT Products ..................................................... 73

Table 11. Time Dependent Nature of SB2 CETL Sludge .................................................................... 84

Table 12. Time Dependent Nature of USC Tank 8 Sludge ................................................................. 84

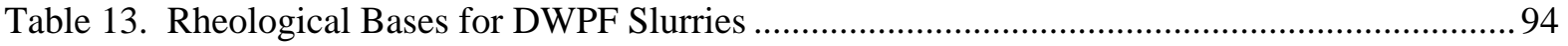

\section{LIST OF ACRONYMS}

ACTL Aiken County Technical Laboratory

CETL Clemson Environmental Technology Laboratory

DWPF Defense Waste Processing Facility

RS150 ThermoHaake RheoStress 150 rheometer

RS600 ThermoHaake RheoStress 600 rheometer

RV20 Haake RotoVisco 20 rheometer

RV30 Haake RotoVisco 30 rheometer

SB2 Sludge Batch 2

SB2/3 Sludge Batch 3 combined with SB2 in Tank 51

SB3 Sludge Batch prepared in Tank 40

SME Slurry Mix Evaporator tank in DWPF

SRAT Sludge Receipt and Adjustment Tank in DWPF

SRNL Savannah River National Laboratory 


\subsection{INTRODUCTION AND BACKGROUND}

Rheological measurements are used to evaluate the fluid dynamic behavior of Defense Waste Processing Facility, DWPF, slurry samples. Measurements are currently made on non-radioactive simulant slurries using two state-of-the-art rheometers located at the Aiken County Technical Laboratory, ACTL. Measurements are made on plant samples using a rheometer in the Savannah River National Laboratory, SRNL, Shielded Cells facility. Low activity simulants or plant samples can be analyzed using a rheometer located in a radioactive hood in SRNL.

Variations in the rheology of SB2 simulants impacted the interpretation of results obtained in a number of related studies. A separate rheological study was initiated with the following four goals:

- Document the variations seen in the simulant slurries, both by a review of recent data, and by a search for similar samples for further study.

- Attempt to explain the variations in rheological behavior, or, failing that, reduce the number of possible causes. In particular, to empirically check for rheometer-related variations.

- Exploit the additional capabilities of the rheometers by developing new measurement methods to study the simulant rheological properties in new ways.

- Formalize the rheological measurement process for DWPF-related samples into a series of protocols.

This report focuses on the third and fourth goals. The emphasis of this report is on the development and formalization of rheological measurement methods used to characterize DWPF slurry samples. The organization is by rheological measurement method. Progress on the first two goals was documented in a concurrent technical report, Koopman (2005). That report focused on the types and possible causes of unusual rheological behavior in simulant slurry samples. It was organized by the sample being studied. The experimental portion of this study was performed in the period of March to April 2004. A general rheology protocol for routine DWPF slurry samples, Koopman (2004b), was drafted in addition to the companion technical report to this document.

Non-radioactive simulants were developed to perform parametric studies that would be too costly to perform with radioactive material. Current simulants adequately duplicate much of the chemistry that occurs in the Sludge Receipt and Adjustment Tank, SRAT, in DWPF. This chemistry includes the pH adjustment with associated conversion of carbonate to carbon dioxide, conversion of nitrite to a mixture of nitrate and oxides of nitrogen, and the reduction of mercury, noble metals, and some manganese. The current simulants are also thought to properly vitrify in the presence of glass frit into a non-radioactive glass simulant of the final waste form.

Simulants mimic real waste in many ways that are important to DWPF processing. Experience gained in the past six years indicates that there remain some physical properties in which it would be desirable for the simulants to match real waste more closely. Simulant processing studies have successfully predicted the acid addition requirement needed for the Shielded Cells SRAT cycle demonstrations performed for Sludge Batch 1A or SB1A, Sludge Batch 1B or SB1B, Sludge Batch 2 or SB2, Sludge Batch 3 alone or SB3, and Sludge Batch 3 as combined with Sludge Batch 2 or SB2/3. Simulants have not accurately matched real waste for the issue of "tackiness" seen with SB1B slurries or the issue of stabilized air entrainment seen in SB2. The rheological properties of simulants, however, appear to be more variable 
than those of real waste. Time dependent behavior has been seen in the controlled shear rate flow curves of some simulant samples. This has not been observed in the limited set of real waste rheological results. Furthermore, simulant properties vary depending on the equipment used to prepare them, even when using the same chemical recipe. Many of these preparation-related issues were investigated in a separate simulant study, Eibling (2004).

The Haake rheometers currently in use support several tests that could be used if time dependent rheological phenomena are observed. Time dependent rheological phenomenon are those where the shear stress required to produce a given shear rate is not constant with respect to time. One such test is the constant shear rate time test (single shear rate for some period of time). Another is the stepped shear rate ramp (step through a small set of discrete shear rates, e.g. 20 shear rates; allow the measured shear stress to stabilize at each shear rate before moving to the next shear rate). The newer rheometers at ACTL can also perform oscillatory measurements. Tests of these types, as well as others, were undertaken in the experimental portion of this program. 


\subsection{APPROACH}

The experimental program was rheological in nature. The second, third, and fourth goals in section 1.0 could not be accomplished without a new approach to rheological measurements. Rheological measurements were made on a set of DWPF simulant samples. These included simulated sludges, SRAT products, and SME products. The set of samples included examples of typical slurries and examples of unusual slurries. The set included examples of rheologically thick slurries and of thin slurries. This breadth in sample characteristics enabled the method development work to span a broad range of potential DWPF slurry rheological properties.

New measurement methods were developed and refined during the experimental work. The major findings covered in this report are the recommended methods for performing the new measurements. Consequently, the discussion in the Approach section will be limited to describing the samples used, and to outlining the individual rheological measurement methods in general terms. Detailed discussion of the finer points of various methods or of their suitability to DWPF samples will be discussed in the method development section, 3.0.

\subsection{Simulant Samples}

There were seven primary test samples. Three were sludges, two were SRAT products, and two were SME products. There were eight supporting samples. These included a viscosity standard, two more sludges, another SRAT product, three SME products, and a variation of one of the primary sludge samples produced by shear-mixing it. The samples, and related visual observations, are given below. Table 1 gives the sample name and summarizes measured non-rheological properties. Clemson Environmental Technologies Laboratory, CETL, sludges were prepared by recipes similar to those used for Optima and University of South Carolina, USC, sludges. There is not a good reference summarizing the preparation and preliminary analysis of the CETL sludges. Table 1 follows the sample summary.

\section{Primary Simulant Samples}

1. SB2/3 CETL SME Product, 155\% Acid, Run 6, 1/16/04 (composite of 2 (or more) 22-L rig products for Slurry-fed Melt Rate Furnace).

This sample was characterized rheologically at the beginning of the program, and was used extensively in evaluating the creep-recovery and controlled shear rate yield point test methods. It was completely consumed before some of the later method refinements could be applied to it. The sample was visibly thick. Bare patches formed on the bob sometimes when it was withdrawn from the sample. Drying was not indicated inside the annular measurement gap, though maybe above the gap. Visually, the slurry seemed to pour thinner from the cup initially sometimes, then thicker, following a measurement. This may indicate some segregation occurred.

2. SB3 CETL SME Product (from SB3A-19, one of the two final flow sheet study runs in support of the SB3 Shielded Cells SRAT/SME simulation; Herman (2004)).

This was one of the samples tested following the preliminary method development with the SB2/3 CETL SME product. In several runs with Taylor vortices, the bob appeared "textured" when it came out of the sample. The bob felt textured when it was rubbed with a gloved finger. The texture was in the form of gritty rings separated by smoother rings. Sample recovered following a rheological measurement actually tested as more solid-rich than fresh sample, 55.23 vs. 53.79 wt. \% TS. Supernate solids, however, were also higher in the recovered material 
(28.13\% vs. 24.6\%). This suggests small, incremental evaporation losses may have been occurring during testing and sample handling.

3. SB2 USC sludge simulant (a blend of Tank 8 and Tank 40 USC sludge simulants; Koopman (2000)). This sample was studied fairly thoroughly in the course of checking out the new rheological test methods. Historically, it was much more viscous than the USC Tank 8 sludge simulant due to the unusually thick USC Tank 40 simulant that was combined with the Tank 8 simulant to produce the blend. This simulant was more orange, i.e. less brown than typical sludges, seemed thinner, and entrained more air bubbles when shaken (was "bubblier") than the chemically-similar CETL SB2 simulant. A 5-mL transfer pipette was used to pull the rheology aliquots from below the surface bubbles. Material that stuck on the bob and beaker walls looked thicker than what was readily poured out following a measurement, but wt. \% solids analysis on the recovered sample indicated that the opposite was true.

4. SB2 CETL sludge simulant, RM-03-131, 8/8/03 (similar in composition to SB2 USC sludge, but made directly, not as a blend of two separate tank simulants).

This sample was extensively tested because of the "hump" in the up ramp portion of the flow curve, which has not been seen in real waste sludges. It was initially tested following the preliminary method development with SB2/3 CETL SME product. There was a large supply, so there were no issues with availability. The sample was relatively thin, easy to mix and pour, and sometimes entrained air bubbles after mixing. Something in sludge simulant stuck to bob and beaker walls, but the sludge didn't stick to the high density polyethylene bottles. It got stickier as it dried. Free pouring sample recovered from the rheometer was not depleted in solids relative to fresh sample. It appeared that there might be sticky zones on the bob, where sludge didn't want to come off using a damp lab wipe. The inside of the rheometer sample beaker was harder to clean than was normal with other sludges.

5. Tank 8 USC sludge simulant, Old Drum 8-1 (a single recipe; Koopman (2000)).

This sample received most of the rheological tests discussed in this report. These provided a set of USC sludge baseline properties. This mixed simulant tended to be bubbly, so it was loaded using a trimmed 5-mL slurry pipette. This effectively kept the large bubbles out of the cup.

6. SB2 Sludge SRAT Product MB3-9/9/03, Mike Stone (CETL-based sludge from M. E. Stone's processing issues work in 2003).

This SRAT product sample prepared from SB2 sludge was given a basic set of tests, but testing was limited because it was quite thin. Further tests were conducted on this sample after it had been decanted to increase the wt. \% total solids, see supporting sample list below. This sample was very bubbly on top after mixing, but not in the bulk, so it was transferred by 5-mL slurry pipette to get aliquots from the bottle to the rheometer sample beaker.

7. SB2/3 CETL SRAT Product (from ARP study, baseline case SRAT cycle; Baich (2003)).

This sample contained small amounts of sand and coal which complicated characterization. It was studied in various concentric cylinder geometries. This sample was not thick. It settled to give about two-thirds supernate after sitting for a day or two. This product did form a clay-like mass after sitting for long periods that needed to be broken up with the drill mixer (hand shaking was inadequate).

\section{Supporting Simulant Samples}

1. National Institute of Standards, NIST, traceable N35 viscosity standard. 
This Newtonian fluid was used to give some comparative results relative to the non-Newtonian slurries, and to perform instrument check-out tests.

2. "Batch 1, Test 6" Optima sludge simulant (essentially equivalent to SB1A).

This sample was introduced to extend the range of sludges to include Optima in addition to USC, CETL, and SRNL. It was only given a couple of tests that would permit some direct comparisons to other sludge simulants. There were distinctly visible orange-white solid particles incorporated in the slurry and seen pouring out with the residue from the beaker.

3. Tank 8/40 (SB2) USC SME Product (from GFPS runs with Frit 200; Koopman (2001)).

This sample was used to test USC vs. CETL SME product characteristics. Only about five different tests were performed to give a quick characterization of it. This slurry was very thick and not bubbly after mixing. It had some agglomerated solid masses in it. It may not have been in the same condition that it was in following preparation in the Glass Feed Preparation System runs (more bubbly when made). A thin dark or black oily-looking ring formed at the top of the gap during the run and poured out of the beaker after the test. This dark material may have been decomposed antifoam. The slurry was clumpy and showed signs of being non-homogeneous, so it was thoroughly blended before rheological testing.

4. SB3 CETL sludge simulant.

This simulant was in the "untrimmed" state. As such, it had very low soluble solids content. It was introduced to check the rheogram hump issue in the SB2 CETL sludge simulant. The sample was not visibly bubbly. It didn’t stick to the bob.

5. SB2 CETL sludge simulant - shear mixed.

This was a variation of the primary SB2 CETL sample (\#4). It was held for about 30 minutes in a Ross high shear mixer. This caused the material to thicken. The test was done to check on whether the hump seen in the original sample could be made to go away by altering the particle size and/or shape through shear mixing.

6. SB2 CETL SRAT Product - decanted.

This was a variation of one of the primary samples (\#6). A portion of this SRAT product was decanted to create a thicker SRAT product than had been available from other archived sources.

7. SB2/3 CETL SME Product, Run 5, 155\% Acid (heel study) and its dilutions, Baich (2004b).

This SME product was added to the test matrix, since Run 6 SMRF feed (Primary Sample \#1) was used up in testing. It was made in the 4-L SRAT/SME rig instead of the 22-L SRAT/SME rig. The largest dilution gave a slurry near the lower end of the DWPF melter feed design basis for rheology. This diluted slurry was observed to partially segregate in a graduated cylinder within ten minutes to a solid-rich bottom layer and solid-poor upper layer. The thicker slurries entrained some air bubbles when shaken/mixed.

8. SB2/3 CETL SME products from acid stoichiometry program (140\%, 155\%, 170\%, and 185\% of stoichiometry; Baich (2004a)).

These four sludge batch 3 SME product simulants were brought in to test controlled stress and oscillating stress results in a group of samples that had already been tested for their controlled shear rate behavior. It was found that the wt. \% total solids had shifted upwards since the initial rheograms were completed 4-5 months earlier, although the $\mathrm{pH}$ remained relatively constant. The samples changed appearance from thick, dark, and not bubbly at $140 \%$ acid to thick, lighter, and not bubbly at $185 \%$ acid. 
Some additional data are included in this report that were obtained as part of other programs. The most significant data are from the measurements on the rheology of SB2 simulants containing depleted uranium. These simulants were made at SRNL, Bannochie (2004). They were processed through a SRAT cycle. Sludge and SRAT products were analyzed using the RV20 rheometer in a radiohood with the MV1 sensor.

Pipette transfers are not generally recommended for slurries with large particles due to the risk of altering the solid distribution in the liquid matrix. The necessary transfers in this test sequence were made using a 5-mL transfer pipette to avoid loading excessive quantities of bubbles into the rheometer. The transfer pipette tip was cut off to leave an opening roughly $1 / 16^{\text {th }}-1 / 8^{\text {th }}$ inch in diameter. This size opening is much larger than the biggest slurry particles, e.g. frit, but small enough to exclude larger air bubbles. This was done to mitigate particle segregation during sub-sampling.

The available physical properties for the above samples are given below. The list in Table 1 is somewhat longer than that above, since individual variations of some samples are listed separately. 
Table 1. Physical Properties of Rheology Slurry Samples

\begin{tabular}{|c|c|c|c|c|c|c|}
\hline & 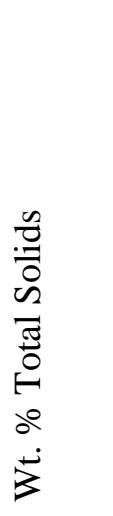 & 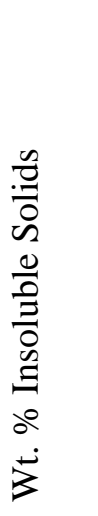 & 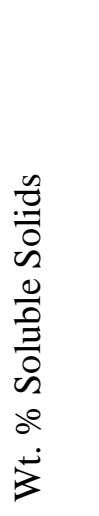 & 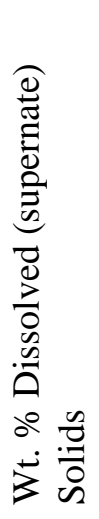 & I & 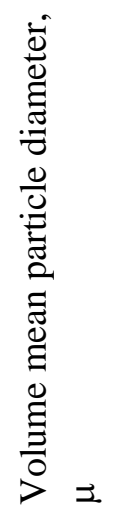 \\
\hline Tank 8 USC Sludge & 17.7 & 15.2 & 2.50 & 2.95 & 10.35 & n. a. \\
\hline Tank 8/40 (SB2) USC Sludge & 16.5 & 13.3 & 3.18 & 3.67 & 11.46 & 4.8 \\
\hline SB2 CETL Sludge & 19.4 & 17.4 & 1.99 & 2.41 & 13.06 & 17.24 \\
\hline Batch 1, Test 6 (SB1A) Sludge & 20.9 & 16.5 & 4.42 & 5.29 & 13.11 & $\sim 4$ \\
\hline SB2 CETL SRAT Product, 9/9/03 & 12.6 & 8.03 & 4.55 & 4.94 & 7.91 & n. a. \\
\hline SB2/3 CETL SRAT Product & 25.4 & 12.8 & 12.6 & 14.5 & 7.43 & n. a. \\
\hline Tank 8/40 (SB2) USC SME Product & 49.3 & 43.3 & 6.02 & 10.6 & 7.98 & n. a. \\
\hline SB2/3 CETL SME Product, Run 6 & $\sim 54.4$ & n. a. & n. a. & n. a. & n. a. & n. a. \\
\hline SB3 CETL SME Product (SB3A-19) & 54 & 37 & 17. & 25 & 7.67 & n. a. \\
\hline SB2 CETL Sludge, shear-mixed & 19.4 & 17.4 & 1.99 & 2.41 & 13.06 & 12.17 \\
\hline SB2/3 SME, Run 5, neat & 50.2 & 37.1 & 13.1 & 20.8 & 6.66 & n. a. \\
\hline SB2/3 SME, Run 5, dilution 1 & 37.9 & n. a. & n. a. & n. a. & n. a. & n. a. \\
\hline SB2/3 SME, Run 5, dilution 2 & 29.5 & n. a. & n. a. & n. a. & n. a. & n. a. \\
\hline SB2/3 SME, Run 5, dilution 3 & 41.5 & n. a. & n. a. & n. a. & n. a. & n. a. \\
\hline SB2 CETL SRAT - decanted & 17.5 & 12.3 & 5.17 & 5.89 & 7.70 & n. a. \\
\hline SB2/3, SME Product, $140 \%$ acid & 55.5 & $\sim 45$ & $\sim 10$ & n. a. & 7.08 & n. a. \\
\hline SB2/3, SME Product, 155\% acid & 52.34 & $\sim 41$ & $\sim 11$ & n. a. & 7.10 & n. a. \\
\hline SB2/3, SME Product, $170 \%$ acid & 53.59 & $\sim 42$ & $\sim 11$ & n. a. & 5.89 & n. a. \\
\hline SB2/3, SME Product, $185 \%$ acid & 53.27 & $\sim 42$ & $\sim 11$ & n. a. & 4.70 & n. a. \\
\hline SB3 CETL Sludge & 17.7 & 17.4 & 0.42 & 0.35 & 11.03 & 9.71 \\
\hline
\end{tabular}

The abbreviation "n.a." in Table 1 stands for not analyzed. 


\subsection{Rheometry}

Rheology is defined as the science of the deformation and flow of matter. Rheometry is the measurement of the deformation and flow of matter.

\subsubsection{Rheometers}

SRNL personnel have been measuring rheological properties of simulant slurries using either Haake RS150 or RS600 research grade rheometers. These instruments are located at ACTL. Radioactive slurry measurements have been made using a Haake RV30/M5 rheometer. This instrument is located in the SRNL Shielded Cells. Some measurements have been made on simulants containing depleted uranium or radioactive samples that can be manipulated by hand using the Haake RV20/M5 rheometer located in an SRNL radioactive hood. The two research grade rheometers were used in this study. Most data were obtained on the RS150.

\subsubsection{Rheometer Sensors}

The primary measurement mode for DWPF slurry samples has been to use the coaxial cylinder geometry operated in the Searle mode (rotating inner cylinder-fixed outer cylinder). This configuration involves a vertical cylindrical beaker, or cup, that holds the slurry sample. A hollow cylindrical sensor, or bob, with a recessed bottom face is moved into the slurry-containing beaker along the vertical centerline axis. The sample flows upwards into the annular region between the walls of the bob and the beaker as the two cylinders are brought to the proper measuring geometry. The rheometer is now in position to perform one of a variety of measurements on the sample. Beakers and bobs for the RS150 and RS600 are interchangeable. These bobs have designations such as Z41, Z38, and Z31 in order of decreasing diameter, Figure 1.

Figure 1. The Z Series Cylindrical Bobs

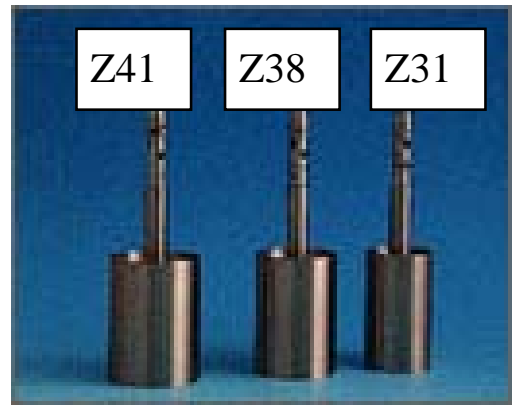

The $\mathrm{Z}$ series bobs are made of titanium instead of stainless steel. The lower mass and inertia of titanium bobs increases their sensitivity and accuracy at low shear rates. Z41 bobs were used for sludge and SRAT product slurry rheology measurements, unless sand and coal were present. In that case some measurements were made with the Z38 bob. Z38 bobs were used for SME product rheology measurements. The Z38 bob is the traditional choice for SME products, but the Z31 bob had seemed to give improved response for the SB2/3 SME products in the acid stoichiometry study, Baich (2004a).

The MV1 bob used in radioactive work is generally similar to the Z41 bob with approximately the same ratio of inner to outer radii of the beaker and bob. The MV1 bob is slightly longer than Z41, $60 \mathrm{~mm}$ vs. $55 \mathrm{~mm}$, and is made of stainless steel. The MV2 bob used in radioactive work is generally similar to the Z38 bob, except that it is also $60 \mathrm{~mm}$ long and stainless steel versus $55 \mathrm{~mm}$ long and titanium for Z38. The measuring beakers are stainless steel for all four rheometers being used for DWPF slurry measurements. 
The measuring beaker was surrounded by a circulating water jacket. The temperature of the jacket fluid was controlled to hold the samples at $25^{\circ} \mathrm{C}$ during all measurements. The rheometers were functionally verified operational using NIST traceable oil standards prior to slurry measurements.

\subsubsection{Rheometer Measurements}

\subsubsection{Controlled Shear Rate Flow Curve}

This is the traditional measurement method for DWPF slurry samples. In this method, the cylindrical bob is accelerated through a predetermined range of rotational speeds, i.e. ramped up, held at the upper speed, and then decelerated back to a stationary position, i.e. ramped down. Times for each step are usercontrolled inputs to the job program, as are the shear rate ranges. The data from the test are referred to as flow curves. The test can be used to check for time dependent behavior simultaneously with obtaining the shear stress-shear rate flow curve data. This makes it a versatile test. While it is useful in identifying the presence of time dependent rheological behavior in a sample, it is not always the best choice for quantifying time dependent behavior.

Ramping of the cylindrical bob speed was done as a linear function of the time, i.e. constant acceleration. If the shear rate was to be ramped from $0 / \mathrm{s}$ to $600 / \mathrm{s}$ over five minutes, then the shear rate was $120 / \mathrm{s}$ after one minute, 240/s after two minutes, etc. Initial rheology measurements used five minutes for both the up ramp and the down ramp durations. Different ramp end points and times were selected for follow-up measurements based on the choice of the cylindrical bob and the nature of the sample. The rheometer measures the torque and angular speed of the rotating bob. Torque was converted to shear stress using a mathematical model that was independent of the rheological behavior combined with information about the measurement geometry. The angular speed was converted to shear rate using a mathematical model that assumed the fluid was Newtonian in behavior.

\subsubsection{Constant Shear Rate Time Curve}

This measurement technique is similar in overall structure to the controlled shear rate flow curve, but the emphasis is on obtaining data during the hold rather than during the up and down ramps. The ramps are very short in duration, while the hold is much longer than in the typical controlled shear rate flow curve. Hold times were typically ten to fifteen minutes, while ramp times were typically 5-20 seconds. Samples were taken to constant shear rates in the range of 100/s to 200/s. The measurement provides a direct test of time dependent behavior.

Lack of time dependence in a sample produces a constant shear stress response at a given shear rate for the duration of the hold. A possible exception occurs in the first minute or less of the hold where inertial effects of the instrument may not have been damped out by the viscosity of the sample. This is more evident in lower viscosity samples. As viscosity decreases, the time to dampen the inertial effects increases. This is taken into consideration when reviewing the data. It can be difficult to distinguish inertial effects from short-lived structural effects within the sample matrix.

\subsubsection{Controlled Stress Flow Curve}

The standard controlled shear rate flow curve measurement, 2.2.3.1, varies shear rate and measures shear stress. The controlled stress variation of the concept varies the shear stress and measures the shear rate. The variation is in the form of a shear stress ramp as a function of time. This is a powerful test for samples suspected of having a yield stress. During the controlled shear rate flow curve, the rheometer drives the stress to whatever level is necessary to deliver the first non-zero shear rate data point. These stresses invariably cause the sample to yield to flow, i.e. are above the true yield stress, since any lower 
stress would not give a non-trivial shear rate. Conversely, in the controlled stress ramp, a stress below the true yield stress gives rise to a zero or trivial shear rate. The sample, however, begins to deform (strain) as the stress applied increases. This deformation can be measured to high precision with the two new rheometers.

Most of the data unique to this measurement are obtained during the up ramp portion. The data can be analyzed for the true yield stress and for the Bingham plastic yield stress and consistency. The true yield stress corresponds to a gel-like to fluid-like transition, while the Bingham plastic yield stress is an empirical extrapolation of high shear rate data back to zero shear rate. The two yield stresses are typically quite different. The total data produced by the measurement can be expanded by adding a down ramp (either controlled stress or controlled rate) to return the sample to zero shear stress. A hold period between the two ramps can be added. The result of these two enhancements produces a controlled stress flow curve data set similar to that in section 2.2.3.1. The raw data can look very similar to the data from the controlled shear rate flow curve when plotted as shear stress versus shear rate. Typically data are also plotted as shear stress versus strain. This type of plot is of negligible value for the controlled shear rate test in 2.2.3.1. A test of this type could replace the standard controlled shear rate flow curve. Only the two cold rheometers (RS150, RS600) have this measurement mode capability, however.

\subsubsection{Small Deformation Yield Point}

This measurement is an attempt to use a controlled shear rate to measure the true yield stress. This measurement is different (reversed) from the controlled stress flow curve ramp at low shear stresses, since shear rate control is used in this test and shear stress is a response. The shear rate ramp used in the controlled shear rate flow curve is adjusted to a very low value (near the limits of the instrument). The cylindrical bob may rotate only $1-10^{\circ}$ in the first 10-30 seconds. As this happens, the sample in the annular gap is strained in much the same way that tensile testing strains a solid sample by pulling it at both ends. Here the stationary end is the beaker wall, and the moving end is the bob. As long as the sample remains solid-like, the rate of rise in shear stress with time is nearly linear.

Once the sample yields, there is a visual transition in a graph of the shear stress versus time from linear to nonlinear behavior. The shear stress at this transition corresponds to the true yield stress of the sample. The test could be attempted on any of the four rheometers, however only the two cold rheometers (RS150, RS600) have the low range sensitivity to justify it for typical DWPF slurries. This test is sometimes performed using a 4-paddle vane sensor instead of a concentric cylinder sensor. This works well for extremely thick samples, and has been used successfully in the Shielded Cells. The insertion of a vane sensor does not disturb the sample nearly as much as the insertion of a cylindrical bob. Time must be allowed for the slurry to return to equilibrium before starting the test.

\subsubsection{Creep-Recovery Test}

This is a very demanding test in terms of time. Each successful test produces one data point. A test consists of imposing a shear stress on the sample for a fixed period of time, e.g. 30-60 seconds. After the stress has been applied for the specified time, it is then removed. The imposition of the initial shear stress produces a strain in the sample. Samples that behave like elastic solids return to their initial position when the stress is removed, i.e. the produced strain, or deformation away from the starting position, is reversed or recovered. Samples that are true fluids recover none of the deformation produced by the imposed stress. A series of tests at various shear stress values is needed to bracket the location of the true yield stress for slurry samples. This is the main drawback of the method. The test requires measurement of the strain on the sample. Only the two ACTL rheometers can perform this test. 


\subsubsection{Oscillation Frequency Sweep}

The remaining tests to be described in section 2.2.3 are fundamentally different from those already given. Those just described rotate the bob in only one direction. The remaining tests involve driving the bob in an alternating clockwise/counter-clockwise oscillatory manner. This introduces adjustable parameters such as the oscillation frequency and the oscillation amplitude in addition to the shear stress, shear rate, strain, and torque.

In the oscillation frequency sweep, the user specifies an oscillation frequency range, e.g. $0.1-10 \mathrm{~Hz}$, and a shear stress to perform this test. The shear stress specified becomes the amplitude of a sine wave function input through the cylindrical bob to the sample. The angular displacement of the bob wall is regulated by the slurry properties. If the sample is rigid, then is oscillates in phase $\left(\delta=0^{\circ}\right)$ with the applied stress (maximum shear stress at maximum displacement). If the sample is entirely fluid-like, then is oscillates with a phase lag to the applied stress $\left(\delta=90^{\circ}\right)$.

The test is usually applied at a stress level below the true yield stress of the sample (not to be confused with the Bingham plastic model yield stress, which is generally much larger). The purpose of the test is to confirm that the sample behaves in a generally solid-like manner over a range of frequencies. This confirmation is needed for the oscillation stress sweep and oscillation time sweep tests, where the oscillation frequency must be specified as an input. The two cold rheometers (RS150, RS600) possess the oscillation mode capabilities for this and the two tests below. The two rheometers in radioactive service have no oscillation mode.

\subsubsection{Oscillation Amplitude (Stress) Sweep}

This test is similar to the oscillation frequency sweep in concept. The difference is that the frequency is held constant and the stress is varied, instead of vice versa. Most testing in this program was done at a frequency of $1 \mathrm{~Hz}$. Higher frequencies gave noisier data, while lower frequencies prolonged the duration of the measurement unduly. A logarithmic distribution was used for the shear stress. A test typically covered 3-4 orders of magnitude in shear stress.

The rheological response to a varying shear stress is obtained in a periodic flow field while the controlled shear stress flow curve is a response to a pseudo-steady state flow field. The two types of data can be fairly similar when plotted as stress versus deformation. Certain physical phenomena, however, can be quite different. One of these is particle alignment. Particles tend to align their long axis parallel to the fluid streamlines in steady flow in order to reduce drag. The regular reversal of flow direction in the oscillatory tests tends to disrupt this type of particle orientation.

\subsubsection{Oscillation Time Sweep}

This test is the oscillation analog to the constant shear rate time curve. A shear stress (not a shear rate) is specified along with a frequency. The sample is then subjected to this oscillating stress for a predetermined time. This was generally 10-15 minutes. Frequency was typically $1 \mathrm{~Hz}$. The shear stress can be chosen above or below the true yield stress of the sample. This is the advantage or enhanced feature of this test relative to the constant shear rate time curve which only obtains data above the yield stress.

The DWPF simulant slurry samples studied in considerable detail were subjected to at least three of these oscillation time sweep tests, i.e. a time sweep series. One test targeted a shear stress that was indicated to be in the region below the true yield stress, i.e. the solid-like region. One test targeted a shear stress that was indicated to be in the region above the Bingham plastic yield stress, i.e. in the definitely fluid-like and yielded region. One or two additional tests targeted shear stresses in the intermediate region between the true and Bingham plastic yield stress values. This oscillation time sweep series permits characterizing 
the time dependence of the sample in three different flow regions. Data obtained indicate the extent of thixotropy or rheopexy at each stress level. This cannot be accomplished with the constant shear rate time curve, since it is unable to make measurements below the Bingham plastic yield stress.

\subsubsection{Rheometer Data Analysis}

The data obtained in the above tests were subjected to one or more types of analysis. These are described individually below.

\subsubsection{Bingham Plastic Fluid Model}

Rheometric data from shear stress-shear rate flow curves are often reduced using a rheological model, or relationship between shear stress and shear rate. One of the simplest of these is the Bingham Plastic fluid model. This model is a two parameter relationship between the shear stress and the shear rate (Newtonian fluids have a one parameter relationship through viscosity):

$$
\text { shear stress }=\text { yield stress }+ \text { consistency } * \text { shear rate, or } \tau=\tau_{0}+\eta_{B} * \dot{\gamma}
$$

The yield stress, $\tau_{0}$, and consistency, $\eta_{B}$, are the two parameters. The consistency is also referred to as the plastic viscosity in some texts. The two parameters are ideally constants for a given sample. This model reduces to the Newtonian fluid when the yield stress is zero. The consistency then becomes equivalent to the Newtonian fluid viscosity. When the yield stress is not zero, however, the consistency is no longer analogous to the viscosity. Instead, the physically analogous quantity to Newtonian viscosity, sometimes referred to as the apparent viscosity, $\eta$, is given by the ratio of the shear stress to the shear rate:

$$
\frac{\text { shear stress }}{\text { shear rate }}=\eta_{B}+\frac{\tau_{0}}{\dot{\gamma}}=\text { apparent viscosity, } \eta
$$

The apparent viscosity of a Bingham plastic fluid decreases with increasing shear rate per equation [2]. This type of fluid behavior is called shear thinning (pseudoplastic). Savannah River Site, SRS, waste slurries typically behave like shear thinning fluids. The apparent viscosity goes to infinity as the shear rate goes to zero. This is a common feature of all models that have a yield stress. The yield stress and the consistency are presumed to be independent of time under shear. The Bingham plastic fluid model is intended to describe steady shear flows such as constant volumetric flow through a duct.

An issue that can arise in making rheological measurements is a departure from laminar flow. This occurs in the form of Taylor vortices when using the concentric cylinder geometry with rotated inner cylinder. Data should be rejected when the shear stress falls below a critical shear stress value that depends on shear rate as given in Smith (2002):

$$
\tau_{\text {critical }}=\frac{\text { density } *\left(R_{o}-R_{i}\right)^{2.5}}{41.3 * R_{i}^{0.5}} * \dot{\gamma}^{2}
$$

$\mathrm{R}_{\mathrm{o}}$ and $\mathrm{R}_{\mathrm{i}}$ are the outer and inner radii of the annular gap respectively. Some of the graphs given in this report will show this relationship in addition to the raw rheological data. More discussion is provided in Appendix A.

Data from the controlled shear rate flow curve can be analyzed using the Bingham plastic equation. The equation can be fitted to the up ramp, to the down ramp, or to both ramps at the same time. If results for 
the up ramp and down ramp are sufficiently different, then the sample may not be responding to shear in a time independent manner. Use of the Bingham plastic equation as a model for the rheological properties may no longer be appropriate. This issue was tracked during method development testing.

Data from the controlled shear stress ramp can also be fitted to the Bingham plastic equation. Use is again limited to samples with minimal time dependence. When time dependence is minimal, the results from the controlled shear stress ramp fit should match the results from both the up ramp and down ramp fits of the controlled shear rate flow curve.

\subsubsection{Thixotropy}

Most tests for time dependent behavior have an empirical, rather than a theoretical, basis. Often, the observation that significant time dependence is present is sufficient. One measurement method requires taking a controlled shear rate flow curve (up ramp, hold, and down ramp). The data is plotted in the conventional shear stress vs. shear rate manner. The observation is made that there is a significant gap between the location of the up ramp data and the down ramp data. The area, $A_{u}$, under the up ramp data is found, and the area, $A_{d}$, under the down ramp data is found. These are then used as follows:

$$
\text { relative thixotropy }=\frac{A_{u}-A_{d}}{A_{u}}
$$

The relative thixotropy by eqn. [4] is positive when the down ramp flow curve is below the up curve. In this case the sample is thixotropic, or thinning with time. If the result from eqn. [4] is negative, then the sample is rheopectic, or thickening with time. This is one reason why some researchers refer to rheopexy as negative thixotropy. The relative thixotropy by eqn. [4] is not a material constant like density. The result obtained depends on the sample properties, how the sample is handled, and on the measurement parameters (especially ramp ranges, ramp times, and hold time). This dependence on measurement parameters provides one of the stronger arguments for standardizing ramp ranges and times, as well as hold times, in the standard controlled shear rate flow curve measurements being performed regularly in SRNL.

The constant shear rate time test and the oscillation time sweep test both produce information about thixotropy. These tests do not require data integrations. The most obvious approach is to compare the property being measured at the end of the test relative to the start of the test. For the constant shear rate time test, the measured property is the apparent viscosity as defined in eqn. [2]. For the oscillation time sweep test, the measured property of interest is the magnitude of the complex viscosity, $\left|\eta^{*}\right|$. To define complex viscosity requires definitions for a series of oscillation test-related terms:

$\begin{array}{lll}\text { Complex modulus: } & \mathrm{G}^{*} & =\tau / \gamma=\mathrm{G}^{\prime}+\mathrm{iG}, \text {, where } \gamma \text { is the measured strain } \\ \text { Storage modulus: } & \mathrm{G}^{\prime} & =\mathrm{G}^{*} \cdot \cos \delta \\ \text { Loss modulus: } & \mathrm{G}^{\prime} & =\mathrm{G}^{*} \cdot \sin \delta \\ \text { Loss factor: } & \tan \delta & =\mathrm{G}^{\prime} / \mathrm{G}^{\prime} \\ \text { Angular velocity: } & \omega & =2 \pi \mathrm{f}, \text { where } \mathrm{f} \text { is the measured oscillation frequency in } \mathrm{Hz} \\ \text { Complex viscosity: } & \eta^{*} & =\mathrm{G}^{*} / \mathrm{i} \omega\end{array}$

where $\mathrm{i}$ is the square root of minus one. Equation [5] contains the Law of Hook (strain linearly proportional to stress).

The rheometer measures strain, $\gamma$, by either: 
- monitoring the cumulative change in the position of the bob relative to the starting position for the constant shear rate time curve

- monitoring the amplitude of the bob oscillation during the oscillation time sweep

The rheometer user sets the frequency of oscillation, $\mathrm{f}$ or $\omega$. The rheometer either measures or applies the shear stress (through torque). The shear stress is driven by a sine wave function in an oscillation test. The rheometer software fits the strain response to a second sine wave function with phase lag. The phase lag of the output strain response wave relative to the input shear stress wave gives $\delta$. This set of measures permits determination of all of the other quantities in eqns. [5] - [10]. The magnitude of the complex viscosity is given by:

$$
\left|\eta^{*}\right|=\sqrt{\left(\frac{G^{\prime \prime}}{\omega}\right)^{2}+\left(\frac{G^{\prime}}{\omega}\right)^{2}}
$$

The argument of the square root is positive for a fluid, since G" > G'. The software reports a complex viscosity even when G” < G', but this has not been explained. A test for thixotropy constructed during the experimental portion of this work was to take a ratio of the final viscosity relative to the viscosity after two minutes, e.g.

$$
\text { relative thixotropy }=\frac{\eta_{\text {final }}}{\eta_{2 \text { minutes }}} \text {, or } \frac{\left|\eta^{*}\right|_{\text {final }}}{\left|\eta^{*}\right|_{2 \text { minutes }}}
$$

The first version of eqn. [12] was used for the constant shear rate time curve, while the second was used for the oscillation time sweep. (Local averages were used instead of point values when the data was noisy.)

\subsubsection{Analysis of Controlled Stress Data}

Strain, $\gamma$, is related to shear rate. Strain is calculated from measurements of the angular movement of the bob. The shear rate is the time rate of change of strain:

$$
\text { shear rate, } \dot{\gamma}=\frac{d \gamma}{d t} \text {; cumulative strain, } \gamma=\int_{0}^{t} \dot{\gamma} d t
$$

These relations are useful in the discussions below.

The preferred analysis for the controlled stress ramp data is done using a graph of $\log \gamma$ vs. $\log \tau$. The Law of Hook, eqn. [5], applies for a solid-like substance, e.g. a yield stress fluid below its yield stress, and a plot of $\log \gamma$ vs. $\log \tau$ is linear with slope one. For a Newtonian liquid, $\dot{\gamma}$ is given by $\tau / \eta$, where $\eta$ is the Newtonian viscosity. If the shear stress is ramped linearly with time, $t$, then $\tau=a * t+\tau_{i}$, where a is a constant related to the final stress and ramp time, $\tau_{\mathrm{i}}$ is the initial stress, and $\mathrm{t}$ is time. The strain is given by the integral in eqn. [13]: 


$$
\gamma=\int_{0}^{t}\left(a t+\tau_{i}\right) / \eta d t=\left(\tau^{2} / 2 a \eta\right)-\left(\tau_{i}^{2} / 2 a \eta\right)
$$

The first term rapidly exceeds the second term, when the initial stress is chosen to be small. At that point, $\log \gamma$ is linear in $\log \tau$ with slope two. Haake technical support has indicated that larger slopes than two are obtained with typical non-Newtonian fluids. The true yielding of a sample should be visible on a plot of $\log \gamma$ vs. $\log \tau$ as a transition from slope one (Law of Hook for solids) to a slope greater than or equal to two (sample behaving as a fluid). Such graphs were generated during method development and are given in the Method Development Findings section.

The rheometer software permits ramping the shear stress either linearly in time as described above, or logarithmically in time, $\tau=\tau_{\mathrm{i}}{ }^{*} \mathrm{e}^{\mathrm{bt}}$. The constant, $\mathrm{b}$, is set by the final shear stress and total ramp time. Performing the integral in eqn. [13] for this case gives

$$
\gamma=\int_{0}^{t}\left(\tau_{i} e^{b t}\right) / \eta d t=\left(\tau-\tau_{i}\right) / b \eta
$$

As the stress difference on the right-hand-side of eqn. [15] increases, the slope of a plot of $\log \gamma$ vs. $\log \tau$ approaches unity. This has the potential to confound the identification of the true yield stress as a transition between slopes. The Findings section, 3.2.2, examines the likelihood of a slurry sample transitioning to a Newtonian liquid (low). In practice, the transition from solid-like to liquid-like was still easy to identify when using the logarithmic ramp option. The most significant observational distinction between the two ramping modes actually arose during the running of a Newtonian viscosity standard. Details are left to section 3.2.1.

Rheometer strain was introduced above as a measured quantity. Some additional detail is needed in discussing the controlled stress ramp strain response. The rheometer strain is defined slightly differently from the strain in a tensile strength test. Strain is a dimensionless quantity, e.g. the change in length per unit length. The software on the two cold rheometers gives the strain as the linear equivalent of the angular displacement of the bob wall, $\theta * R_{i}$, relative to the annular gap spacing, $R_{0}-R_{i}$, where $R_{0}$ and $R_{i}$ are the radius of the cylindrical cup and cylindrical bob, and $\theta$ is the angular displacement from the initial position in radians. The annular gap is approximately in the range of $1-2.5 \mathrm{~mm}$. A value of one for strain means that the bob has turned through an angle such that the bob wall has moved about 1-2.5 mm. The common bob radius is of order $20 \mathrm{~mm}$. Therefore, the bob has rotated roughly 1-8 ${ }^{\circ}$ (depending on specific dimensions). Slurry samples typically yield before the strain reaches unity. A strain of unity represents a stretching of about $40 \%$ for a point initially on the wall relative to a point at the same initial angular position on the bob. This is a relatively extreme strain except for polymeric systems.

The yield stress is usually obtained by fitting the region of slope one to a power equation, $\gamma=c_{1} \tau^{\mathrm{c} 2}$. Then the region with slope two (or greater) is fitted to a second power equation. The two equations are solved simultaneously for stress and strain, i.e. the intersection of the two fits. The stress obtained is taken as an accurate approximation to the true yield stress of the sample. This can also be done graphically.

As will be discussed further in section 3.2, most of the DWPF simulant slurry samples had a transition in slope as stress increased. This occurred at a shear stress well below the traditional Bingham plastic yield stress. A second transition, however, was seen on the $\log \gamma$ vs. $\log \tau$ plot at higher stresses. After yielding, the slope was typically of order ten for a range of stress values. The slope then decreased dramatically to something closer to two. This was followed by another transition to a slope much greater 
than two. This second transition occurred at a shear stress that was very similar in magnitude to the traditional Bingham plastic yield stress. This transition was at large values of the strain, corresponding to something of order one full rotation of the bob. This much strain would easily break a rubber band, i.e. this is not a yield stress in the sense of the conversion of a weakly gelled solid-like phase into a fluid-like phase. The same power equation analysis can be applied to the second transition that was applied to the first transition. The result is a yield stress similar to that obtained by fitting shear stress-shear rate data to the Bingham plastic equation, but it is not identical. Examples will be given in section 3.2.

The oscillation stress sweep gives different data than the controlled shear stress flow curve, but some of the data is very similar in appearance. The software drives the bob through an oscillation in the form of a sine wave with an amplitude convertible into units of shear stress. The actual position of the bob is in the form of a sine wave with a phase lag and an amplitude convertible into units of strain. The same analysis described above for the controlled stress ramp strain response can be applied to the oscillation stress sweep. The transition at the true yield point is as apparent, or even more apparent. The transition corresponding to the Bingham plastic yield stress is less apparent. The strain goes up very quickly in the controlled stress ramp after the Bingham yield, because the bob is spinning freely. The strain goes up much less quickly in the oscillation stress sweep, because the bob continues to oscillate about the point of zero strain. The bob was not observed to make even one full rotation before reversing during an oscillation at high stress, though such a situation is not inconceivable.

In addition to the shear stress-shear rate plot used in most historical work, and the $\log \gamma$-log $\tau$ plot described above, data from the controlled stress ramp can be plotted in the form log of the apparent viscosity, $\eta$, versus $\log \tau$. This plot has been used in the literature to spot potential occurrences of a phenomenon referred to as slip. Slip is generally taken to be the situation when the fluid in the gap totally or partially loses contact with the rotating bob. When this occurs, there is a significant reduction in the shear stress. For a homogeneous sample, such as a liquid polymer, this plot starts with a high constant viscosity (the "Newtonian plateau") and then transitions into a nearly linear region of falling viscosity with increasing shear (the "power law fluid" region). The "power exponent" in the power law is given by the slope of this plot. Slip reportedly manifests itself as a premature drop in the shear stress followed by an eventual return at higher shear stress to the true fluid (recovery from the slip condition). The sample appears to be at a new, lower Newtonian plateau following the onset of slip. The plot begins to resemble steps down as stress increases. Most of the DWPF slurry samples tested had this feature, but supporting tests did not indicate that slip was the reason for it.

In addition to the $\log \gamma$-log $\tau$ plot described above, data from the oscillation stress sweep comes in terms of the storage and loss moduli, G' and G”. These moduli are also obtained during the oscillation frequency sweep and the oscillation time sweep. The storage modulus, G', represents the solid-like response of the sample, while the loss modulus, G”, represents the fluid-like response. When G' > G”, the sample is considered to be more solid-like. When G" > G', the sample is considered to be more liquid-like. Plots of G' and G' versus shear stress show the transition from solid-like to liquid-like behavior quite clearly. The transition occurs at approximately the true yield stress, as defined by the low stress transition in slopes on the $\log \gamma-\log \tau$ plot.

The arctangent of the loss factor is $\delta$, given by Arc-tangent(G”/G'). This parameter is $0^{\circ}$ for a true solid, and is $90^{\circ}$ for a true liquid. Slurry samples generally had $\delta \sim 10-15^{\circ}$ at low stress, and $\delta \sim 75-85^{\circ}$ at high stress. $\delta$ followed an S-curve shape as stress increased. The point where $\delta$ began to rise quickly on a $\delta$ $\log \tau$ plot seemed to correspond to the true yield stress obtained by the other methods. There was also a later dip in $\delta$, followed by a second rise. The shear stress corresponding to the bottom of the dip seemed to be approximately the same as the Bingham plastic yield stress. The $\delta$ term stayed well above $45^{\circ}$ 
throughout this Bingham yield stress region, i.e. the slurries were clearly liquid-like before and after this transition.

\subsubsection{Analysis of Small Deformation Yield Point Data}

Data from this measurement are usually plotted as shear stress versus time. The shear stress tends to rise linearly with time initially (before it has yielded). Yielding is seen as either a maximum in this plot, or, at least a transition to a more nearly horizontal line. The test is designed to occur at constant shear rate. This shear rate is typically so low that the software is either unable to control it or to measure it accurately (probably the latter). The maximum shear stress is taken as the true yield stress of the sample. No other parameters are obtained.

\subsubsection{Analysis of Creep-Recovery Data}

Creep-recovery data is plotted as strain versus time (there is no shear rate, and shear stress is constant). Strain should increase linearly with time in the creep portion of the test (especially if below the yield stress). Strain may or may not recover once the stress is removed. Recovery is seldom $100 \%$, however 80-90\% recovery is expected if the stress applied was not sufficient to cause the sample to yield. ThermoHaake RheoWin software determines some quantities from the data, such as the zero stress viscosity (Newtonian limit) and a recovery time constant. The software determines the percent recovery as well. This can vary depending on the time allocated for the recovery portion of the measurement. The creep-recovery technique proved to be less user-friendly than others that could give an approximation of the true yield stress, so no more discussion will be given on analysis.

\subsubsection{Analysis of Oscillation Frequency Sweep Data}

The analysis of this data has the primary goal of establishing the suitability of a given frequency for making the oscillation stress and time sweeps. As such, the log-log plot made is of the two moduli, G' and G" as a function of frequency. Whichever is greater should remain greater over the range tested, and specifically in a neighborhood around the proposed frequency for the other tests.

The complex viscosity, $\eta^{*}$, is also obtained as a function of frequency. The Cox-Moore Rule proposes that $\eta(\dot{\gamma}) \cong\left|\eta^{*}(\omega)\right|$, where shear rate and frequency are presumed to be equivalent. This is typically a good approximation at high shear rates. Data obtained were, however, typically at stress levels below the true yield stress. Application of this rule only gave insight into the apparent viscosity at very low shear rates $(<5 / \mathrm{s})$. It did not appear to be feasible to extract the equivalent data to a controlled shear rate or shear stress flow curve using the Cox-Moore Rule.

\subsubsection{Analysis Matrix}

The following table summarizes the number of each of the tests outlined in section 2.2.3 that were performed on each sample. 
WSRC-TR-2004-00334

Revision 0

Table 2. Summary of Tests Performed by Sample and Test Type

\begin{tabular}{|c|c|c|c|c|c|c|c|c|}
\hline Sample & 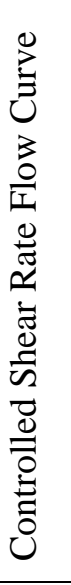 & 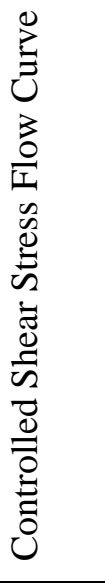 & 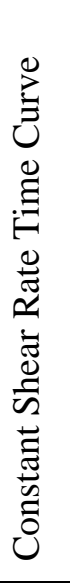 & 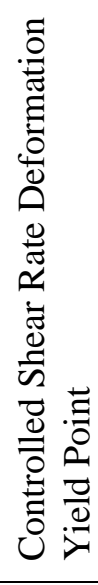 & 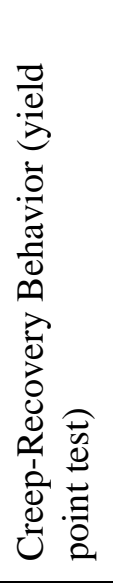 & 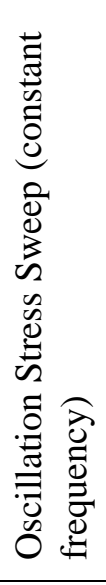 & 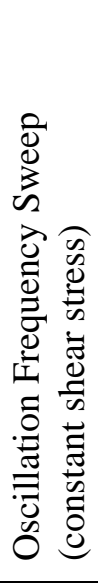 & 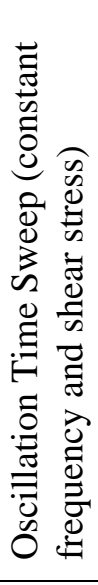 \\
\hline N35 NIST Viscosity Oil Standard & 5 & 3 & 1 & 1 & 2 & 4 & 4 & 0 \\
\hline Tank 8 USC Sludge & 1 & 3 & 1 & 0 & 2 & 3 & 1 & 3 \\
\hline Tank 8/40 (SB2) USC Sludge & 1 & 5 & 2 & 4 & 4 & 2 & 1 & 4 \\
\hline SB2 CETL Sludge & 9 & 3 & 3 & 4 & 4 & 2 & 6 & 4 \\
\hline Batch 1, Test 6 (SB1A) Sludge & 0 & 1 & 1 & 0 & 0 & 0 & 0 & 0 \\
\hline SB2 CETL SRAT Product & 2 & 2 & 1 & 0 & 0 & 1 & 1 & 0 \\
\hline SB2/3 CETL SRAT Product & 8 & 2 & 1 & 0 & 0 & 1 & 1 & 0 \\
\hline Tank 8/40 (SB2) USC SME Product & 2 & 1 & 1 & 0 & 0 & 1 & 0 & 0 \\
\hline SB2/3 CETL SME Product & 3 & 4 & 2 & 12 & 13 & 6 & 5 & 4 \\
\hline SB3 CETL SME Product (SB3A-19) & 5 & 4 & 3 & 2 & 1 & 3 & 1 & 3 \\
\hline SB2 CETL Sludge, shear-mixed & 1 & 0 & 0 & 0 & 0 & 0 & 0 & 0 \\
\hline SB2/3 SME, neat + dilutions (4) & 8 & 1 & 2 & 0 & 0 & 1 & 0 & 0 \\
\hline SB2 CETL SRAT - decanted & 1 & 1 & 2 & 0 & 0 & 1 & 0 & 0 \\
\hline SB2/3, 4 Acid-level SME Products & 0 & 1-2еа & 0 & 0 & 0 & 1 ea & 0 & 0 \\
\hline SB3 CETL Sludge & 1 & 0 & 0 & 0 & 0 & 0 & 0 & 0 \\
\hline
\end{tabular}




\subsection{RHEOLOGICAL METHOD DEVELOPMENT FINDINGS}

Significant results of experimental measurements will be covered in this section. These results were extracted from a larger body of data. The full data set can be found in lab notebook WSRC-NB-200400009

Four rheometric tests were found to be the most useful. These were the controlled shear rate flow curve (traditional test), the controlled shear stress flow curve, the constant shear rate time curve, and the oscillation stress sweep. This block of four tests gives:

1. The familiar controlled shear rate flow curves from which the Bingham plastic yield stress and consistency are calculated.

2. A check on the Bingham plastic fit from the corresponding controlled shear stress flow curve data.

3. A direct assessment of thixotropy from the separation of the up and down flow curves in the controlled shear rate data.

4. A confirmation/clarification of thixotropic behavior from the constant shear rate time curve.

5. An estimate of the persistence of thixotropic trends from the constant shear rate time curve.

6. A measure of the true yield stress of the slurry from the low shear rate data in the controlled shear stress flow curve.

7. A confirmation of the true yield stress of the slurry from the low shear rate data in the oscillation stress sweep.

8. A third estimate of the Bingham plastic yield stress from the second transition in the oscillation stress sweep delta parameter.

9. Confirmation that the solid-like to liquid-like transition is occurring at the true yield stress through the relative values of the storage modulus, G', and loss modulus, G”.

10. Data at shear rates from $\sim 10^{-4} / \mathrm{s}$ to $10 / \mathrm{s}$ from the controlled shear stress flow curve that are more likely to be accurate than comparable data from the controlled shear rate flow curve (this region is important to sedimentation and to gravity draining calculations).

Contained in this block of information was the insight to explain much of the unusual behavior observed in some of the slurry samples that have been run on the rheometers in the past two years.

The discussion that follows is sorted by rheological test method. The direct rotational methods will be discussed first. This includes the controlled shear rate flow curve, constant shear rate time curve, controlled stress flow curve, and several other tests. The discussion of the oscillatory methods follows that of the rotational methods. Examples of the data obtained by each method, and the analyses performed are given. Variations of the individual methods will be discussed as well. All of the methods have adjustable parameters. The results (consequences) of changing some of these independent 
parameters on the data obtained will be reviewed. The complementary report, Koopman (2005), discusses the application of the methods to some of the samples that gave unusual rheological behavior.

\subsection{Controlled Shear Rate Flow Curve Studies}

This test received considerable scrutiny, since it has been the mainstay of the SRNL rheology program for many years. There has been no formally standardized method of performing this measurement. The approaches have fallen into a fairly narrow window. This study allowed certain rheometer parameters to move into regions typically not used to evaluate the impact on the results. Essentially, the test has been performed as follows:

- Choose a cup and bob for the slurry sample, in particular select an annular gap that will not be subject to bridging, or jamming, by the largest particles in the slurry. Select a gap that is ten times larger than the largest particle if data are available.

- Load a sample aliquot into the instrument beaker and then bring the cylindrical bob into the sample until the annular gap is filled.

- Hold the sample until a temperature equilibrium is reached with the temperature control jacket.

- Ramp the shear rate from 0/s up to a maximum shear rate (anywhere from 100/s to 1000/s). Take about five minutes for this ramp. Ramp linearly in time.

- Hold the sample at the maximum shear rate for a period of time (generally 30-60 seconds).

- Ramp the shear rate down from the maximum shear rate to $0 / \mathrm{s}$. Take the same length of time used in the up ramp portion of the job.

- $\quad$ Remove the sample. Load a fresh sample aliquot, and perform a duplicate measurement to check reproducibility of the data.

This study considered variations in:

- the shear rate range maximum

- the time for the ramps both as faster ramps to the same maximum shear rate and as shorter ramps to a lower maximum shear rate

- the effect of pre-shearing the sample in the rheometer at a constant and fairly high shear rate prior to initiating the up ramp

- the data obtained from running the flow curve program consecutively on the same sample aliquot

- the impact of the profiled bob and cup combination on difficult data (the profiled pair is less susceptible to slip at the wall, since it has a series of vertical machined grooves unlike the smooth surfaces of the typical cup and bob combinations)

- the interaction of the bottom of the bob with the bottom of the beaker (by varying the spacing between the bottom of the bob relative to the bottom of the beaker) 
- the effect of changing the fraction of insoluble solids

All data presented below were taken with the temperature control bath set to deliver $25^{\circ} \mathrm{C}$.

\subsubsection{Geometric Effects}

The typical set-up for a sludge or SRAT product on the cold rheometers is to select the Z41 cylindrical bob. This bob is positioned $3 \mathrm{~mm}$ above the bottom of the beaker by default during the measurement. The typical set up for a SME product is to select the somewhat smaller diameter Z38 cylindrical bob. The larger annular gap is preferred to cope with the larger frit particles in SME products. This bob is positioned $8 \mathrm{~mm}$ above the bottom of the beaker by default during the measurement. Both the Z41 and Z38 bobs have smooth surfaces, as does the beaker. There is also a PZ38 cylindrical bob. This bob is not smooth-walled. It has vertical grooves running the length of the cylindrical wall. It has its own cup, which is also grooved on its inner wall. The "P" stands for profiled, because the surface has a noncircular profile. The numbers in the vendor bob names correspond to the approximate diameters of the cylindrical bobs in $\mathrm{mm}$. Additionally, the factors that convert angular speed and torque into shear rate and shear stress are the same for both the Z38 and PZ38 beaker-bob combinations.

During the experimental work, the impact of changing bobs and of changing the distance off the bottom of the cup was examined. Figure 2 shows the up and down ramp flow curve segments for a SME product run using the Z38 bob with an $8 \mathrm{~mm}$ bottom gap versus a $12 \mathrm{~mm}$ bottom gap below the bob.

Figure 2. SB2/3 SME Product, Run 5, Heel Study: Effect of Bottom Gap

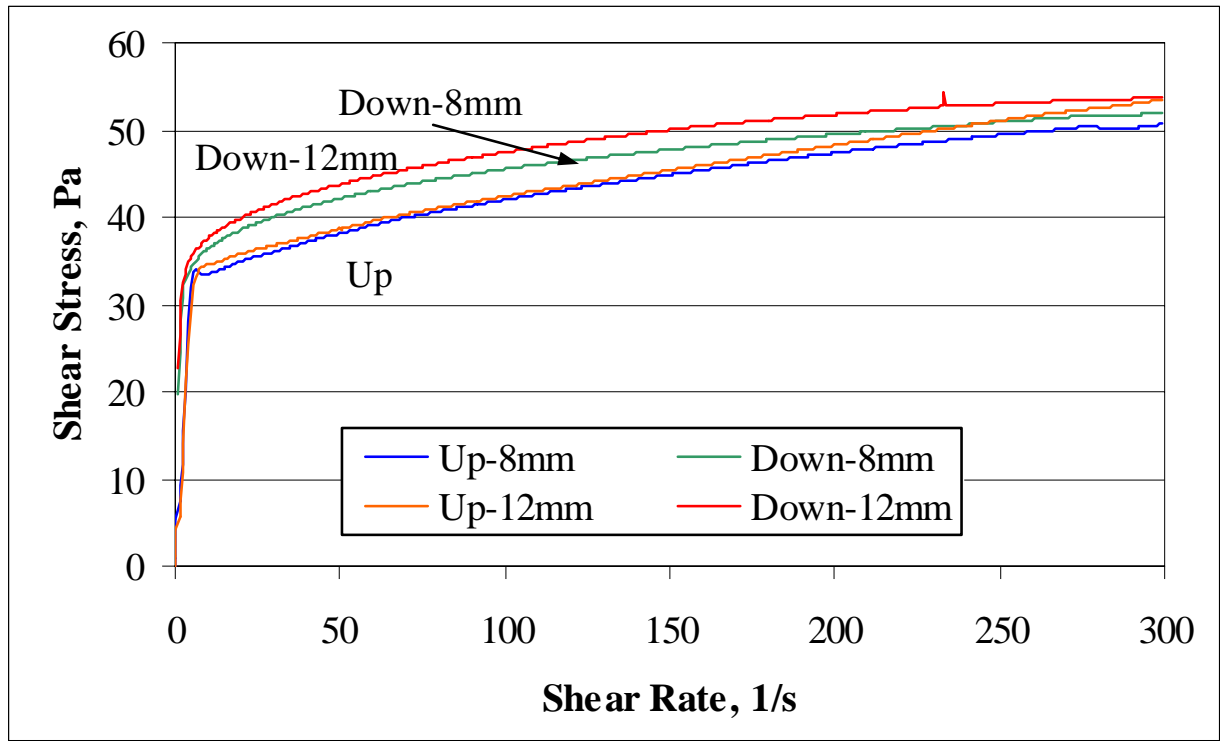

The effect of the bottom gap was fairly minimal on the results obtained. Note that this SME product is exhibiting mildly rheopectic tendencies, since the later down ramp flow curve segments lay above the earlier up ramp flow curve segments. The apparent rheopectic behavior may be due to particle segregation effects in the gap. The shear rate was ramped to 300/s over five minutes, held there for one minute, and then ramped down to $0 / \mathrm{s}$ over five minutes. These are the most common choices for the three segment times in prior work. Aliquots were held 3-7 minutes in the ready position (bob at the gap) before initiating the up ramp to obtain thermal equilibrium and some structural build-up. 
SB2/3 SRAT product based on the CETL simulants was tested using the Z41 bob at two different depths, Figure 3. This sample was also tested at 8-mm and 12-mm depths using the Z38 bob. The larger gap Z38 bob was used because of the presence of small amounts of relatively coarse sand and coal. These particles were much larger than typical sludge particles, although they were similar to frit particle sizes. Figure 3 shows the effect of the larger particles on the Z41 up flow curves. There are a series of stress spikes during the initial portion of the up ramp flow curve. It is hypothesized that the larger slurry particles eventually disperse and align with the flow streamlines. Once this happens, there are no more stress spikes. The annular gap obtained with the Z41 bob is not 30-50 times the mean size of the sand and coal particles as recommended by Alderman and Heywood (2004a). The annular gap may be 30-50 times the mean size of the sludge particles plus sand and coal particles, but the rule of thumb probably wasn't developed from data containing such a bimodal distribution of particle sizes.

Figure 3. SB2/3 CETL SRAT Product by Z41: Effect of Bottom Gap

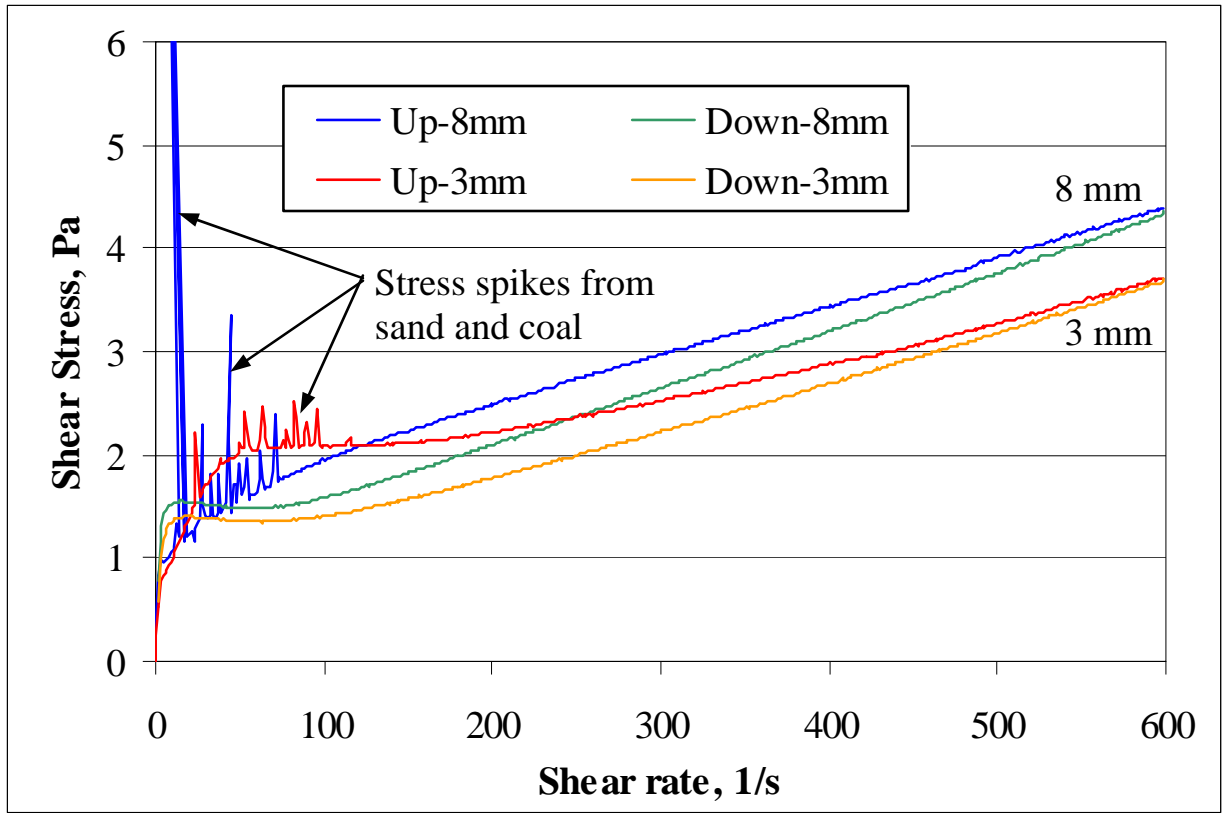

The effect of the bottom gap on the data was again fairly minimal. The 8-mm data appeared somewhat thicker, but the sample potentially dried somewhat in the three weeks between the two measurements. Slow drying was an issue with many of the samples that were used throughout the test program. Shear rate ramp times were five minutes, and the hold time at 600/s was one minute. 
Figure 4. SB2/3 CETL SRAT Product by Z38: Effect of Bottom Gap

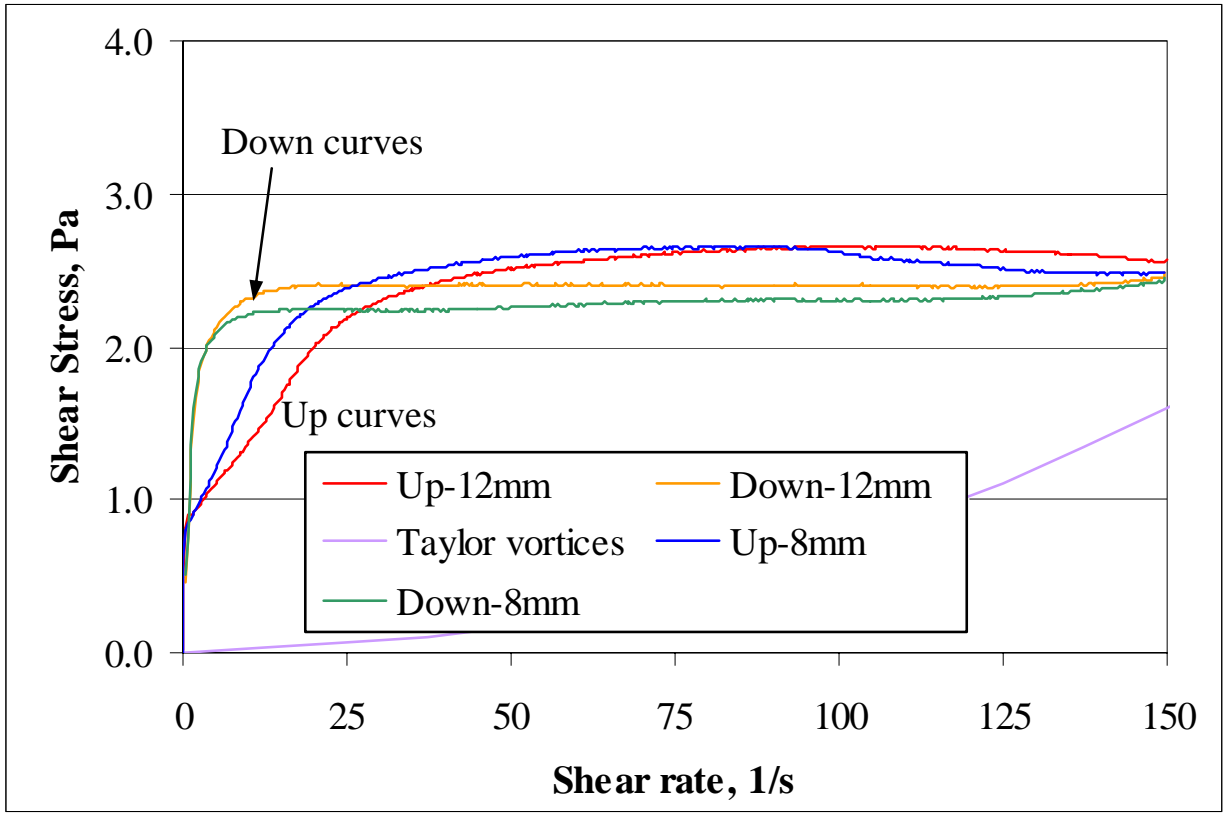

There was only a minimal impact from raising the Z38 bob an additional $4 \mathrm{~mm}$ off the bottom of the cup. The switch from Z41 to Z38 between Figure 3 and Figure 4 clearly had a much larger impact on the shape of the flow curve. Another example of the impact of changing the bob size is given in Figure 5.

Figure 5. Impact of Bob Selection on Flow Curve for SB2 CETL Sludge

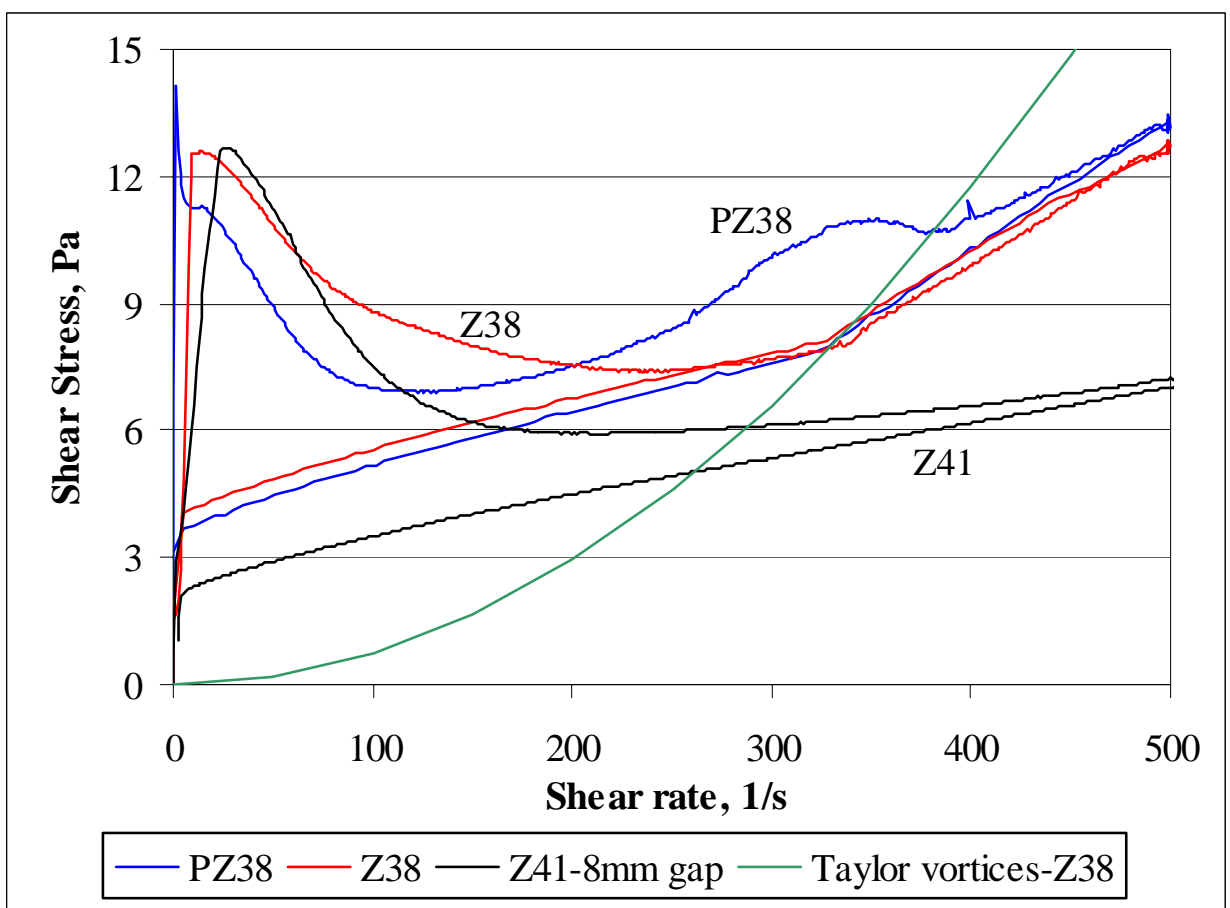


The effect of different bobs on the SB2 CETL sludge impacted the location of the smoother down curves, but had only a minimal impact on the up curve hump. The profiled PZ38 bob pulled the hump back to nearly zero shear rate. The conclusion from this portion of the testing was that the issues being seen in the rheograms were not due to geometric effects within the rheometer. The Taylor vortices were only seen in the two Z38 geometries and not in the Z41 geometry as expected. The onset of Taylor vortices for the Z41 bob comes at higher shear rates than were tested for this sample. Examples are given in Appendix A.

\subsubsection{Effect of Independent Parameters on the Controlled Shear Rate Flow Curve}

The controlled shear rate flow curve has several adjustable parameters that can potentially affect the quality of the data being obtained. The initial shear rate is generally taken to be $0 / \mathrm{s}$. The maximum shear rate at the end of the up ramp has been taken anywhere from 100/s to 1000/s. The time to ramp from $0 / \mathrm{s}$ to the maximum shear rate is typically five minutes, regardless of the maximum. This leads to variations in the time rate of change of the shear rate during measurements that could have undetermined effects on the results being generated.

SB2 SRAT product based on CETL sludge was selected for initial testing. The initial test applied a normal shear rate ramp from $0 / \mathrm{s}$ to $600 / \mathrm{s}$ in five minutes, followed by a one minute hold at $600 / \mathrm{s}$, and then a ramp down to 0/s over five minutes. An accelerated test was run over the same shear rate range with one minute ramps and a 12 second hold, Figure 6.

Figure 6. SB2 CETL SRAT Product: Effect of Five-fold Faster Loop

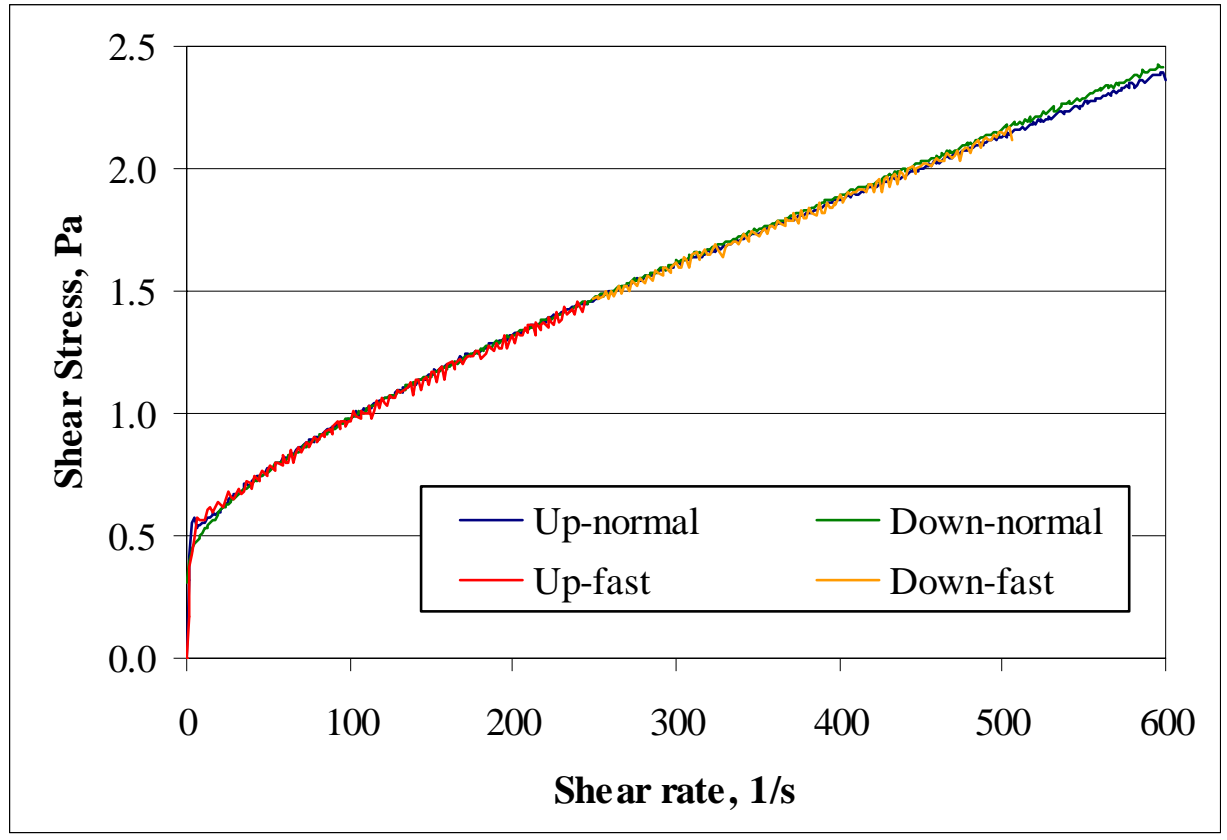

The noteworthy features of Figure 6 are listed below:

- The normal speed test showed no signs of time dependence (nor did the constant shear rate time curve). The up and down ramp flow curves were virtually superimposed.

- The high speed test was indistinguishable from the normal speed test. 
- This SRAT product was a bit thin, with a Bingham plastic yield stress of about 5 dynes $/ \mathrm{cm}^{2}(0.5$ $\mathrm{Pa})$.

- The flow curve from $100 / \mathrm{s}$ to 600 /s was nearly linear. This region would be well-fitted by the Bingham plastic equation.

If all DWPF simulant samples behaved like this, then this study would not have been necessary. Also, samples of this type have rheological properties that at most depend only weakly on the shear rate ramp parameter selections. This is good. It implies that historical data on well-behaved samples would be comparable even if different measurement methods were used to generate the various rheometric data sets.

The effect of maximum shear rate was studied on the SME product from SB3A-19. This was one of the two final simulant flow sheet studies prior to the SB3 Shielded Cells qualification run, i.e. it did not contain the SB2 portion of the DWPF SB3 feed. Figure 7 gives data from four runs. One run ramped from $0-300 / \mathrm{s}$ in 300 seconds. The other three ramped from $0-100 / \mathrm{s}$ in 100 s, i.e. the ramp rates were identical.

Figure 7. Reproducibility of Flow Curves for SB3 CETL SME Product, Z38

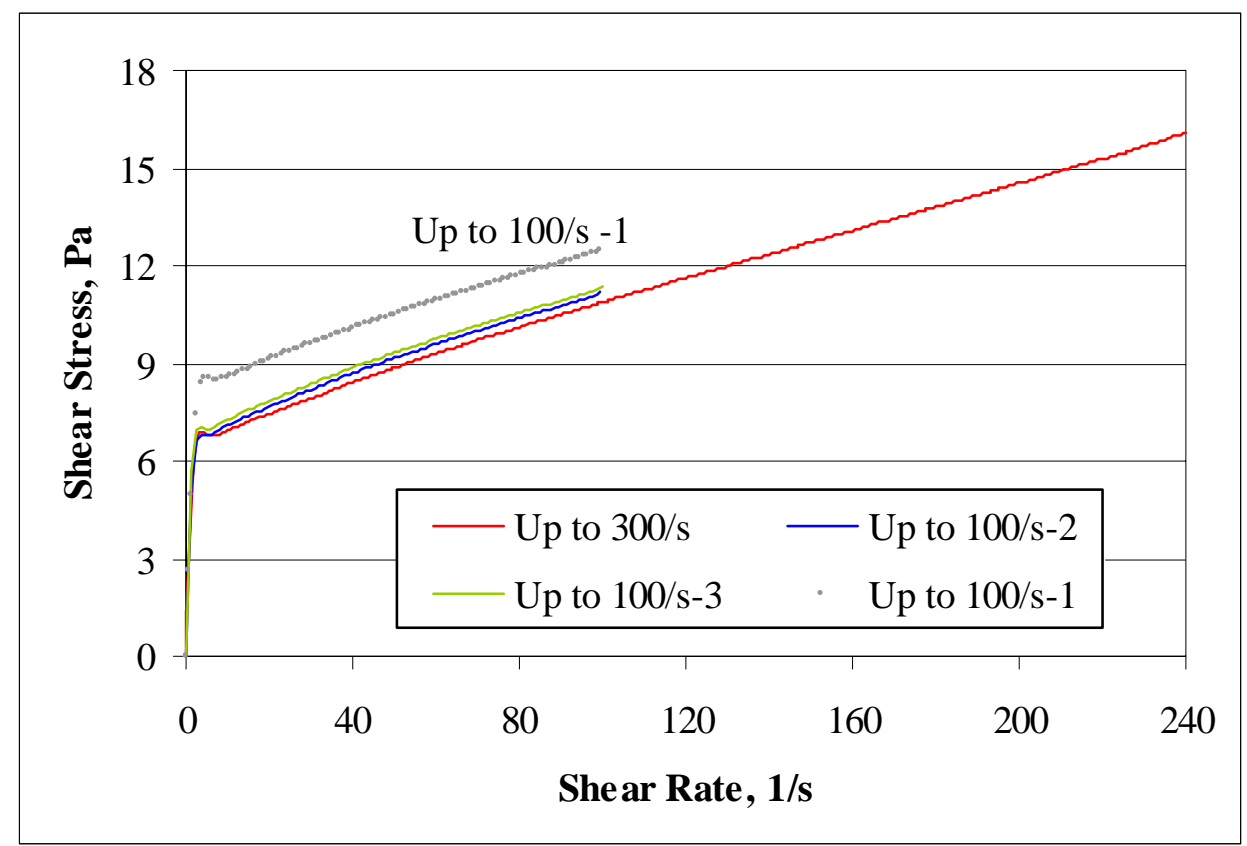

Virtually identical data was obtained in three out of four runs over the interval from 0-100/s as expected. The initial up ramp to 100/s is an example of what can happen when working with slurries. In this case, the rheometer had been charged from an initially 200-mL sized aliquot from a larger bottle. The sample aliquot had been nearly used up, and apparently because of less than perfect mixing and some potential drying, the sample had thickened. Upon taking a fresh aliquot from the larger bottle, the results again matched those obtained initially. The other data indicate that, with care and adequate sample volume, very high reproducibility is possible.

The SB3A-19 slurry indicated rheopexy in the 0-300/s flow curve, Figure 8. This was a 5-1-5 minute job for the up, hold, and down segments respectively. Fresh sample was loaded and rerun to 200/s using 100 
seconds for the up and down ramps and two seconds for the hold. The result was fairly dramatic as seen in Figure 8.

Figure 8. SB3 CETL SME Product: Effect of Faster Loop with Reduced Range, Z38

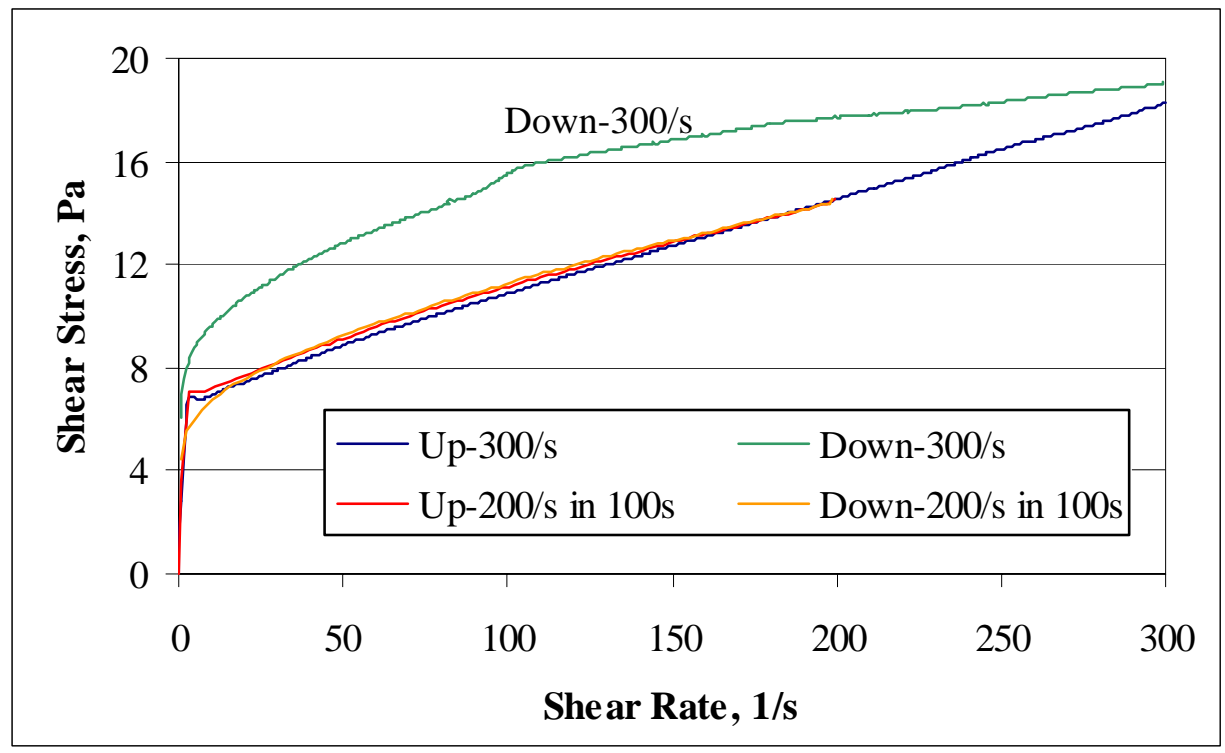

Taylor vortices were not an issue here. The shear stress would have had to have been below $8 \mathrm{~Pa}$ at 300/s to have come close. What appears to have happened is that the faster loop measurement on a reduced range avoided conditions that would have led to the rheopectic behavior seen in the standard curve. The ramp rate of the faster loop is only double that of the standard loop $\left(2 \mathrm{~s}^{-1} / \mathrm{s}\right.$ versus $\left.1 \mathrm{~s}^{-1} / \mathrm{s}\right)$. The reduced range up and down flow curves overlay each other, and they are similar to the standard up flow curve.

This data might support arguments that the maximum shear rate should be kept lower for SME products than is indicated by the region of Taylor vortices. More support comes from the next figure, Figure 9. Here are two ramps for 100 seconds each with maximum shear stresses that differ by a factor of two.

Figure 9. SB3 CETL SME Product: Reduced Shear Rate Range - Constant Duration, Z38

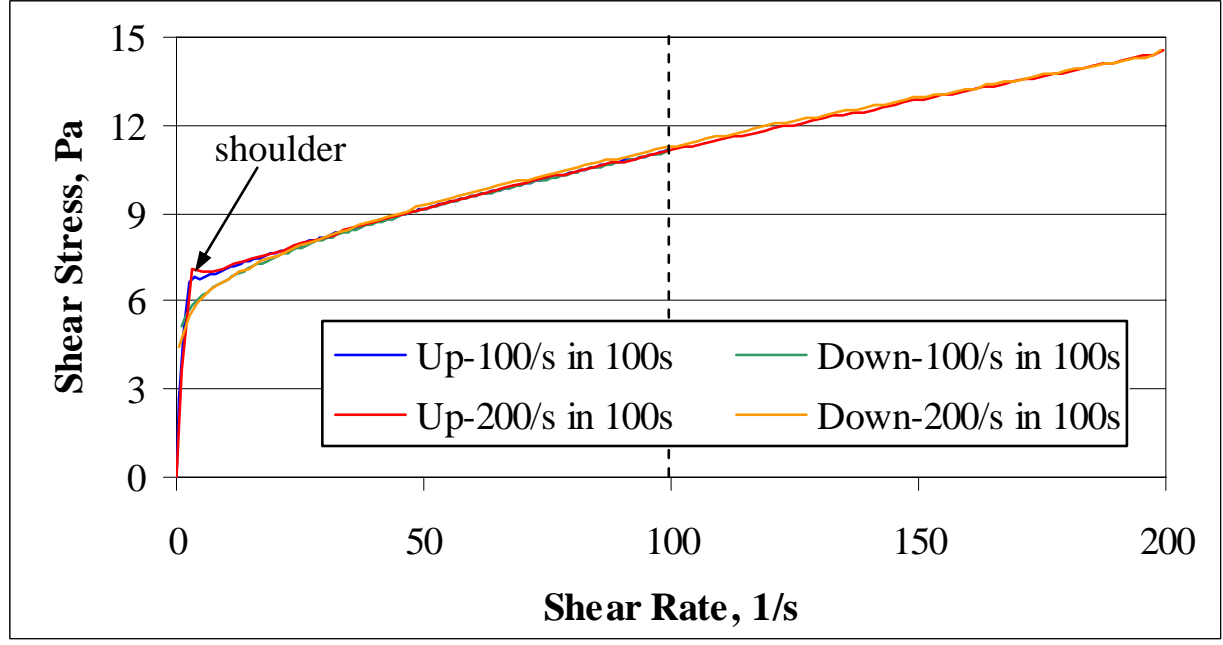


Notice how both up ramps capture a small shoulder at about 5-15/s shear rate, and how the data in the entire region from $0-100 / \mathrm{s}$ is superimposed for the two up ramps as well as for the two down ramps. Negligible time dependence is indicated by the flow curve data. Some part of that is undoubtedly due to short duration of the measurements, but the rest is likely due to the reduced measurement ranges.

\subsubsection{Cross-over Loops}

Several systems studied in this work had a down ramp flow curve that intersected the up ramp flow curve at some intermediate value of the shear rate that was well away from $0 / \mathrm{s}$. These flow curves resemble lopsided figure-8's. Figure 10 shows an example for SB2/3 CETL SRAT product.

Figure 10. Examples of Cross-over Flow Curves for SB2/3 SRAT Product

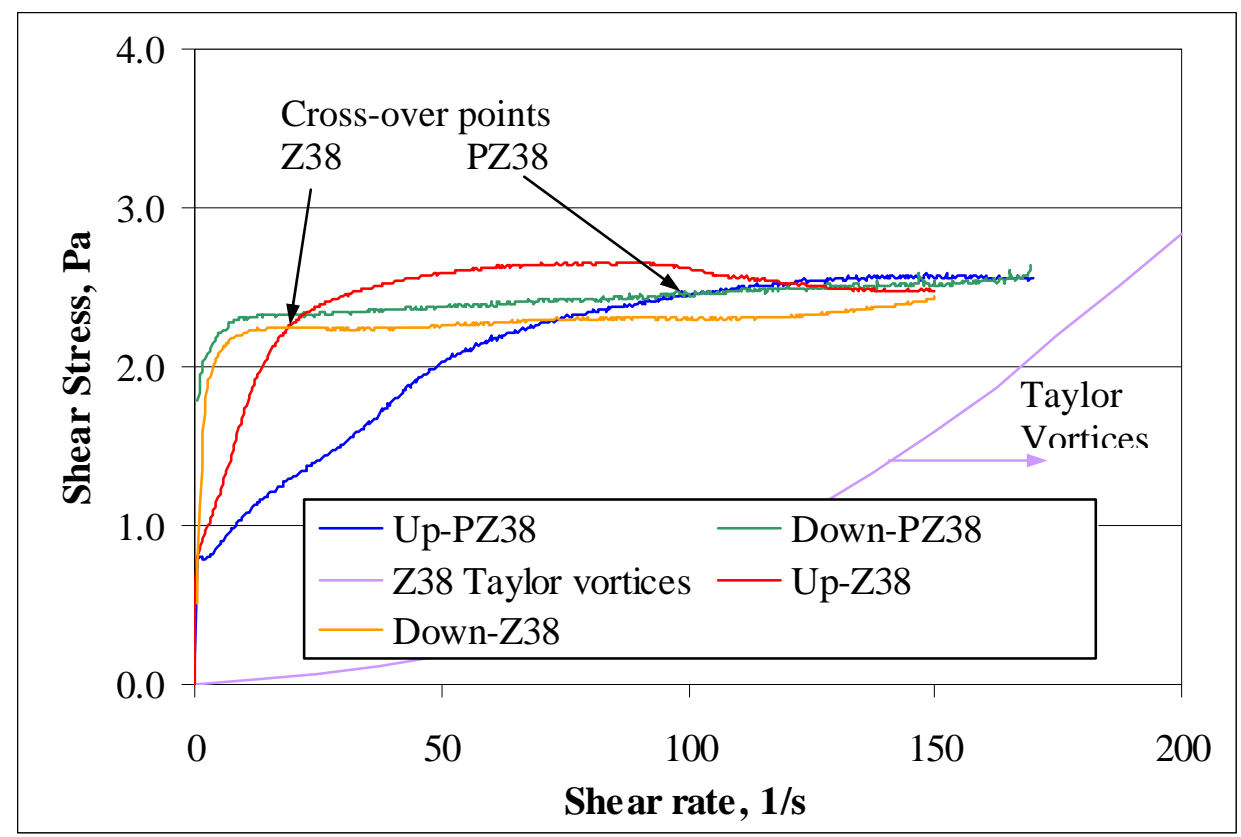

Data in Figure 10 also show that cross-over was impacted by choosing a profiled (PZ38) versus a smooth (Z38) bob. Figure 11 shows two standard flow curves for the Tank 8/40 (SB2) USC SME product. 
Figure 11. SB2 USC SME Product Cross-overs, Z38

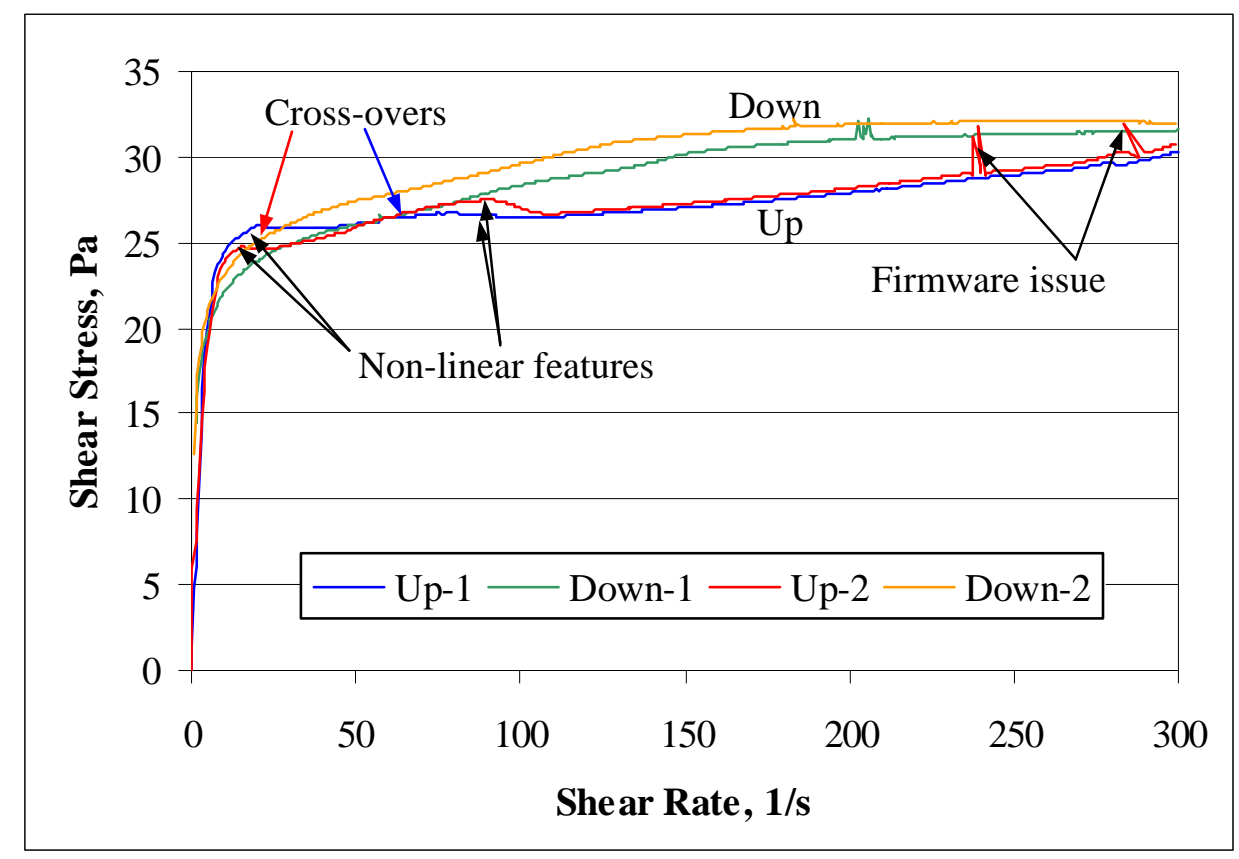

It is interesting that some of the non-linear features in the ramps are reproduced, such as the up ramp region between 15/s and 120/s, even though they initially appear to be abnormalities, or at least departures from the expected smooth curve shape of rheometer data. There was a small amount of electronic noise (looks like little stress spikes) that was not cleaned up, but was a firmware issue, not a sample issue. This issue has been corrected in the instrument since the data were taken. This sample was also a little unusual in the constant shear rate time curve at 100/s. It thinned slightly over the first six minutes then thickened slightly over the next nine minutes. The controlled shear rate flow curve data could be interpreted as thickening at high shear rates and thinning at low shear rates. One interpretation that I would put forward is that there may be two (or more) different phenomena at work in the DWPF simulant slurries. This sample may be an instance where two of the phenomena are of roughly similar significance with respect to the rheological behavior, while the more general case is that only a single phenomenon is dominant.

Initial SME product data was obtained on slurries that exceeded the DWPF design basis window for yield stress. A SME product was obtained in sufficient quantity that a series of dilutions could be completed on it until rheological properties were obtained in and near the lower end of the DWPF design basis. This data is presented in Figure 12, since it bears on the subject of flow curve cross-over. The nominal DWPF yield stress-consistency SME product range is included for comparison. 
Figure 12. Impact of Dilution on SB2/3 Run 5 SME Product, Z38

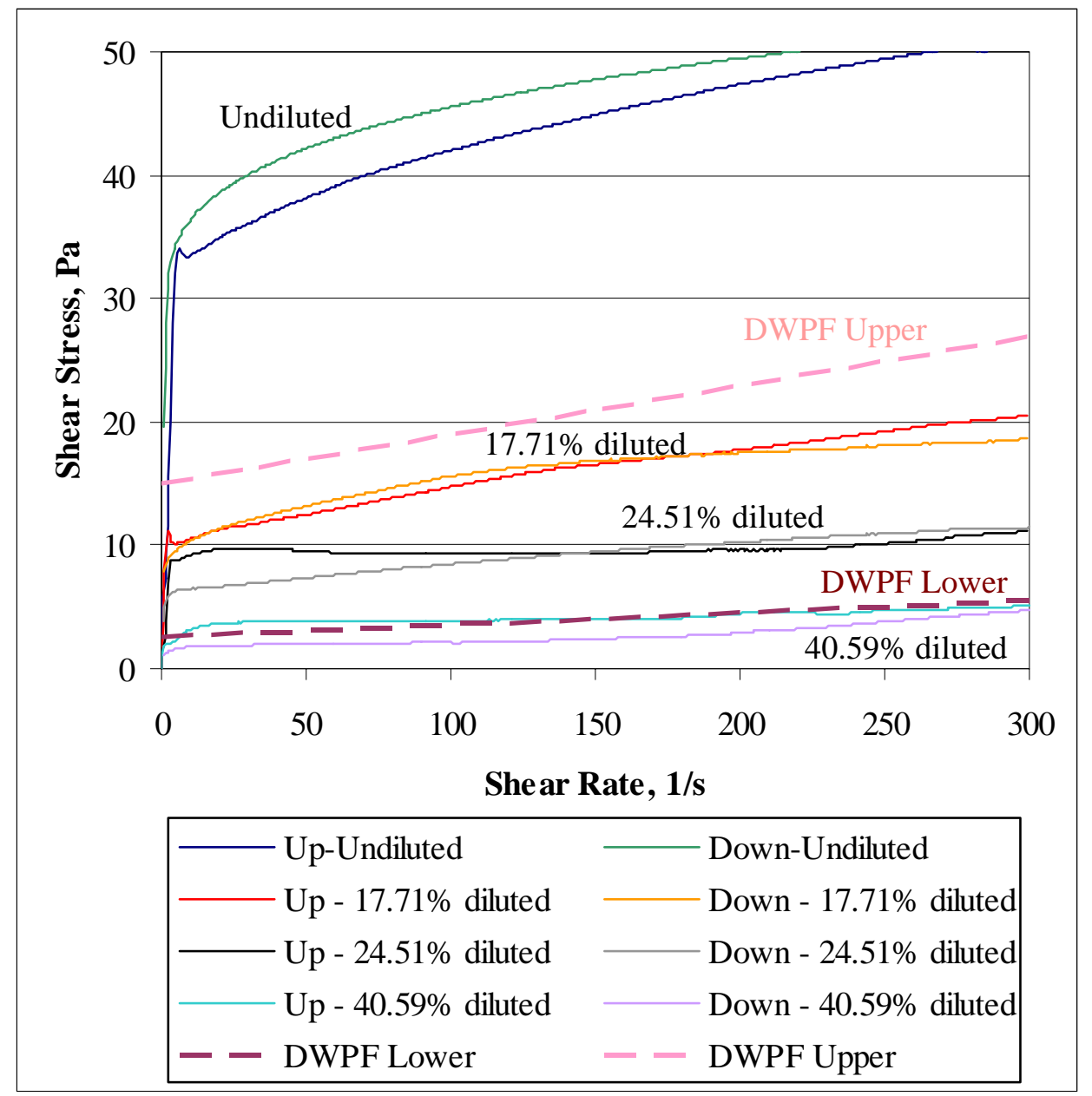

The undiluted sample behaved in a mildly rheopectic manner in its flow curve (top). The most diluted sample (40.59\% by mass added water) showed evidence of mildly thixotropic behavior (up curve above down curve - bottom). The two intermediate dilutions both have a flow curve cross-over point. These data suggest that the cross-over phenomena may be related to the apparent thixotropy and rheopexy being seen in the simulant samples. Cross-over may be occurring at solids loadings that are in the transition region between thixotropic and rheopectic behavior. This would be consistent with a "balance between competing phenomena" interpretation as proposed above, and would also link the cross-over phenomenon in with the other time dependent issues with rheology, rather than putting it in a separate category. These data are also consistent with the general description of slurry rheology in Alderman and Heywood (2004a) that indicates that the type of rheological behavior observed can change as the solids concentration changes. In this paper, shear-thickening behavior occurred at higher solids loading than shear-thinning behavior.

\subsubsection{Development of Multi-Curve Measurement}

Time dependent rheological behavior had been previously identified in flow curves for some of the samples being tested. This finding led to the in-depth study on samples with the most unusual behavior, Koopman (2005). During testing with the unusual samples, it was found that the changes with time under shear were diminishing when consecutive flow curve measurements were performed on a sample. This was interpreted to mean that the flow curve was approaching a rheological steady state. To perform a 
multiple flow curve test, the sample was loaded and run normally using the appropriate controlled shear rate flow curve program. At the end of the initial flow curve measurement, the sample was retained in the instrument. Another controlled shear rate program was run (often the same program again) on the sample.

The goal was to attempt to drive the slurry toward its rheological steady state condition. This is the condition that the slurry has after a long time under shear, i.e. it is not the start-up behavior associated with putting an unsheared slurry into motion. This latter situation would be a much more difficult problem to characterize, since unsheared slurries tend to settle into a solid rich phase and a supernate phase. It was necessary with some of the samples to measure quickly enough to avoid any issues related to segregation or settling of the slurry particles in the annular gap of the rheometer.

Data are presented below for sludges, SRAT products, and SME products. Figure 13 gives data for CETL SB2 sludge simulant. This unusual simulant was studied extensively, and the full set of significant results can be found in the companion report, Koopman (2005). Data were obtained at a temperature of $25^{\circ} \mathrm{C}$ using the $\mathrm{Z} 41 \mathrm{bob}$.

Figure 13. Impact of Consecutive Flow Curve Routine on SB2 CETL Sludge, Z41

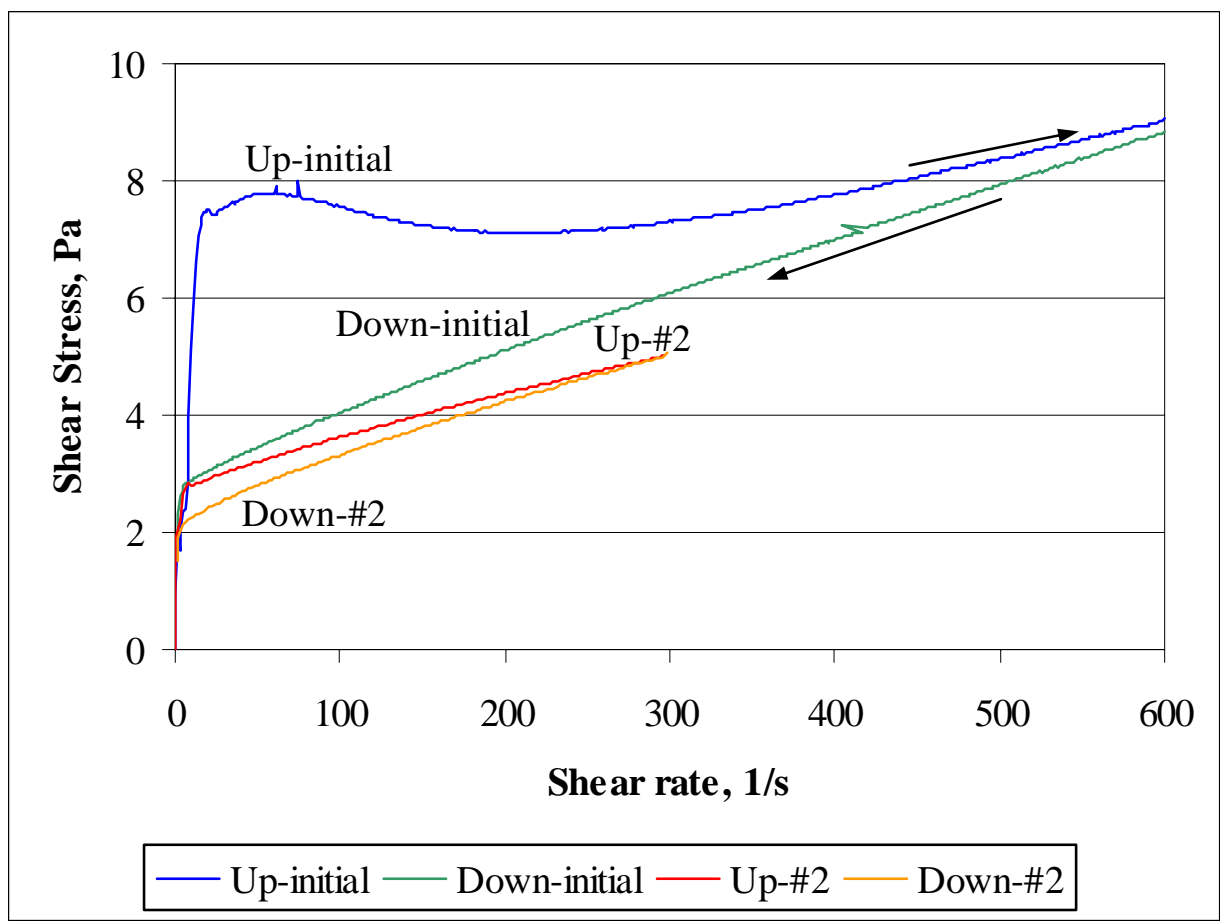

The second flow curve range on the sample was kept shorter intentionally. The ramp rate was $2 / \mathrm{s}$ per second for all ramp segments, so the elimination of the hump in the up ramp was not due to changing the ramp rate. This sample still showed diminished signs of time dependence after the second flow curve was completed. This was indicated by the small separation between "Up-\#2" and "Down-\#2" at low shear rates. This is the point of maximum difference in time between the up and down ramps for a given flow curve. A third repetition of the flow curve may have been necessary to capture the steady state flow curve. 
Another pair of sequential flow curves was generated using SB2/3 SME product slurry. The example is for Dilution 3 of the SME Product from SB2/3 Run \#5 (17.71\% diluted). These data are given in Figure 14. Data were obtained at $25^{\circ} \mathrm{C}$ using the Z38 bob. The sample had been diluted with de-ionized water to lower the yield stress. The flow curves were still well above the region where Taylor vortices form. "Up-Dilution 3-2" was the initial up ramp on the sample, while "Down-Dilution 3-3" was the second (final) down ramp.

Figure 14. Consecutive Flow Curves for Diluted SB2/3 CETL SME Product, Z38

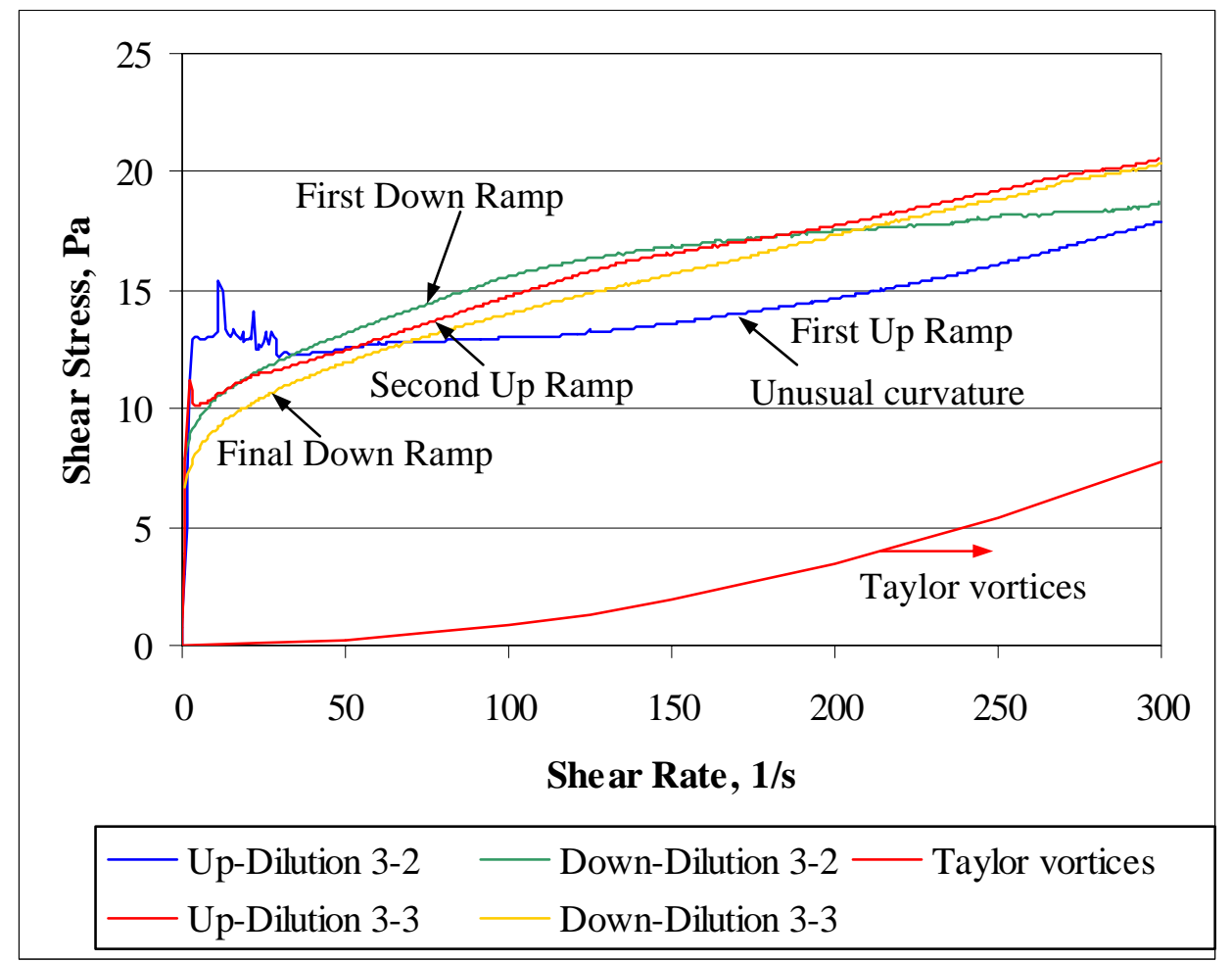

The initial up ramp flow curve was very irregular at shear rates less than 35/s, and then it followed an unusual upwardly curved path until the hold at 300/s. The initial down ramp, the second up ramp, and the second down ramp were curved downwards. Each successive ramp was closer to the previous ramp as the sequence progressed. This slurry was inside the nominal DWPF yield stress range of 2.5-15 Pa (25150 dynes $/ \mathrm{cm}^{2}$ ). The data trended toward a Bingham plastic yield stress of about $10 \mathrm{~Pa}$.

Another SB2/3 SME product was tested using consecutive flow curves, Figure 15. In this case, there were two up ramps but only one down ramp. The test was discontinued after the second up ramp. Data were obtained at $25^{\circ} \mathrm{C}$ using the $\mathrm{Z} 38$ bob. 
Figure 15. Impact of Consecutive Flow Curves on SB2/3 CETL SME Product, Z38

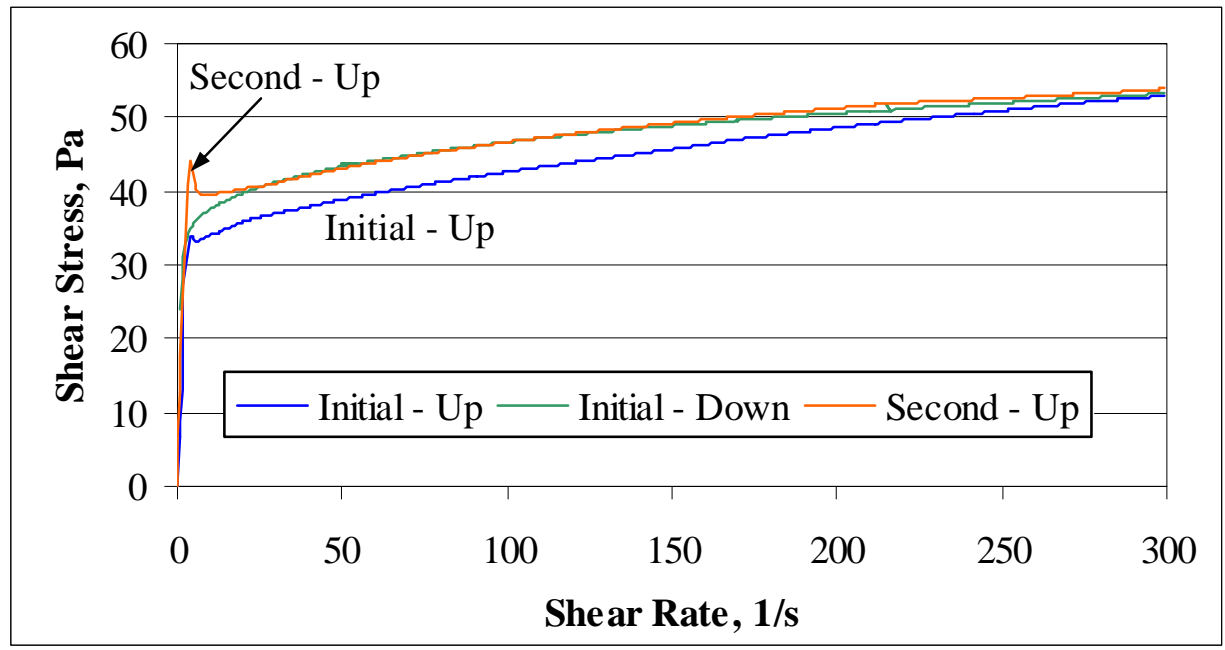

This was SB2/3 CETL SME product from Run 6 at 155\% stoichiometry. This measurement showed that the down ramp from initial flow curve was close to the steady state shear behavior, since the second up ramp was nearly identical to it. The most likely conclusion would be that this slurry reached rheological steady state during the initial down ramp. This slurry was thicker than the one in Figure 14. This may impact how quickly the slurry reaches rheological steady state. There was no evidence of trapped air bubbles in the slurry in the rheometer or of significant solids segregation.

The next two graphs, labeled Figure 16, show data obtained in a study that occurred a few weeks before the method development testing, Bannochie (2004). A series of six sludge batch 2, SB2, sludge simulants had been prepared at five different concentrations of depleted uranium. There were two versions of the nominally $7.5 \%$ DU sludge, as well as versions at nominally $0 \%, 3.75 \%, 11.25 \%$ and $15 \%$. Rheological measurements were made on all six sludges as well as the SRAT products produced from each sludge as part of that program. The first data shown are for a pair of measurements made on the SRAT product from the second version of the $7.5 \%$ DU sludge simulant. These flow curves were obtained using the RV20 rheometer in a radiohood. The MV1 bob was used (analogous to the Z41). Temperature was controlled at $25^{\circ} \mathrm{C}$. 
Figure 16. Consecutive Flow Curves on 7.5\% DU SRAT Product
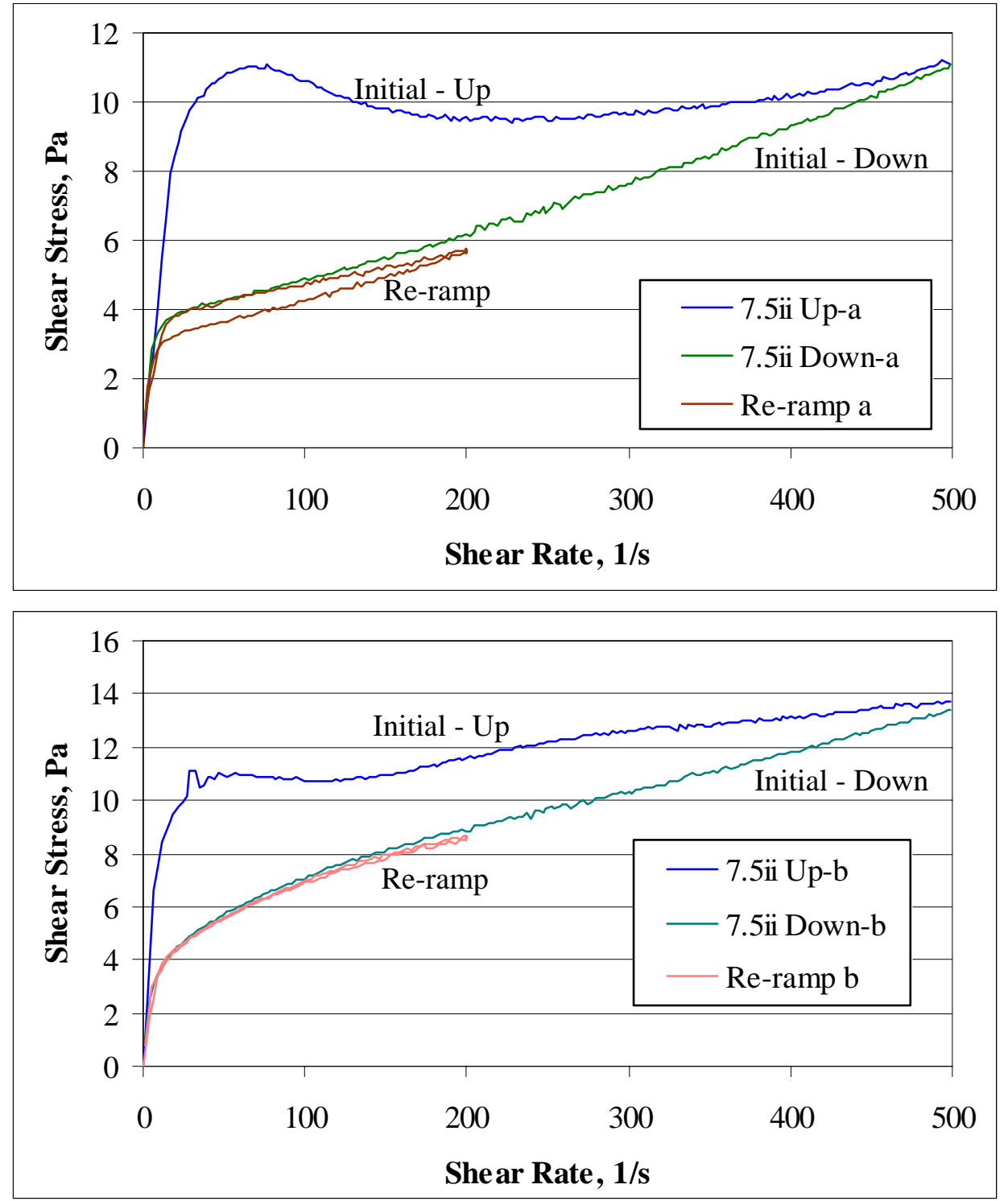

The re-ramp included both an up and a down ramp. Ramp rates were 100/s per minute for both the initial flow curve and for the re-ramp. The ramp range was dropped from 0-500/s to 0-200/s for the re-ramp. This was done to shorten the time available for any settling of solids. The re-ramp data in the lower graph of the two in Figure 16 are nearly indistinguishable from the initial down ramp suggesting that rheological steady state was achieved.

The next graphs, Figure 17, were made on the sludge simulant with $15 \%$ DU in the solids. The ramp rates, ranges, bob, etc. were the same as used for the 7.5\% DU SRAT product. The re-ramp included an up ramp only. The traces Up-4A and Up-5A are the second up ramps. These flow curves were also obtained using the RV20 rheometer. The MV1 bob was used (generally similar to the Z41 geometry on the RS150 and RS600). Temperature was controlled at $25^{\circ} \mathrm{C}$. 
Figure 17. Impact of Consecutive Flow Curves on 15\% DU Sludge
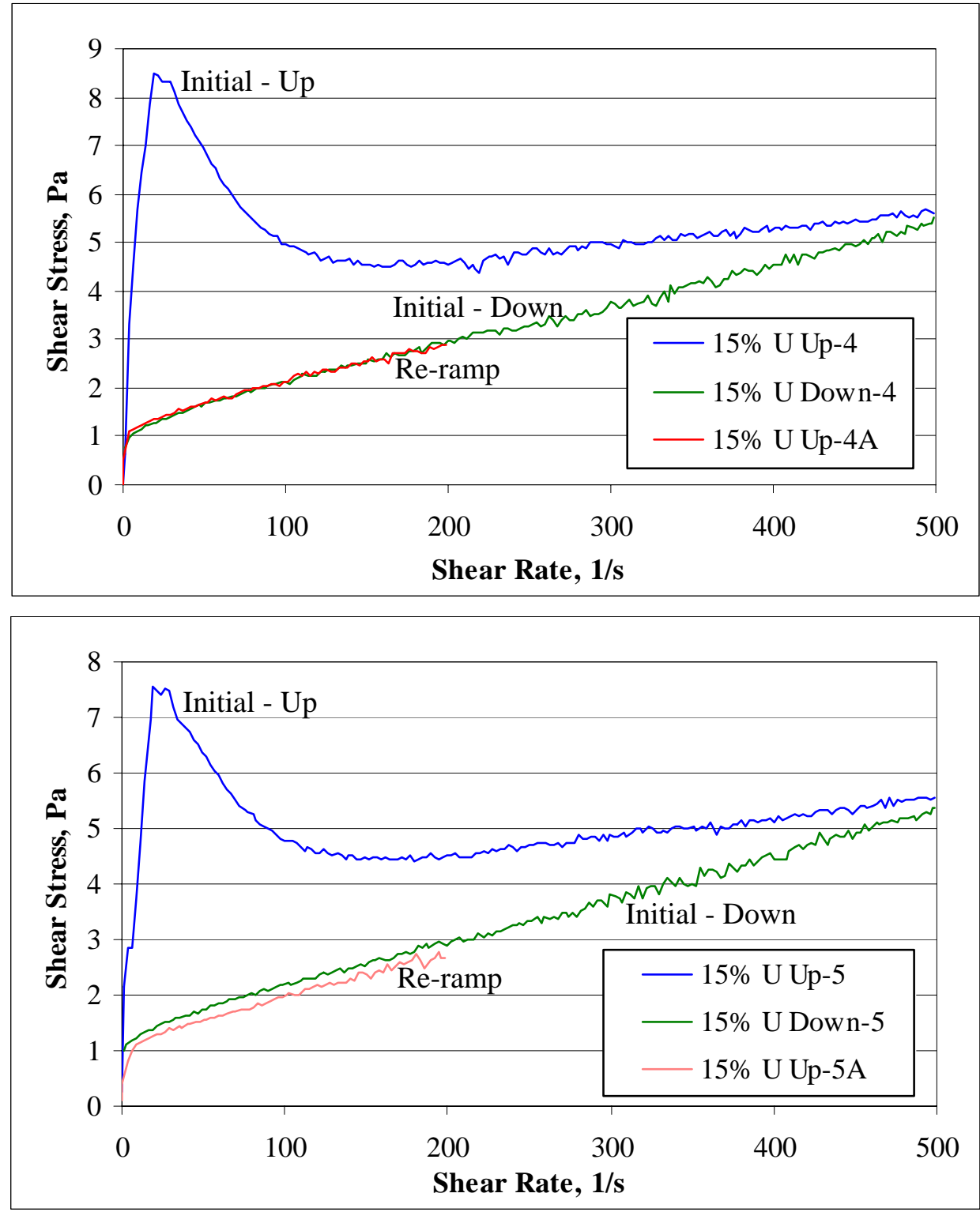

These data can be used to estimate how quickly the sludge slurry reached a rheological steady state. This sample was fairly close to steady state after the initial flow curve down ramp based on the proximity of the second up ramp to the initial down ramp data.

\subsubsection{Impact of Taylor Vortices}

It was observed that some SME products as well as a few sludge and SRAT products had been ramped into the region of Taylor vortices. The solution for the equations of motion used to analyze rheometric data assumes one dimensional laminar flow in cylindrical coordinates. Taylor vortices are a multidimensional flow. When the rheometric data enters the region of Taylor vortices, the high shear rate data must be excluded from subsequent analyses. 
The situation is potentially even more complicated for slurry samples. The one-dimensional flow laminar pattern is horizontal angular flow. Taylor vortices introduce radial and vertical velocity components into the annular region holding the sample. Koopman (2005) includes a figure showing the flow field which resembles a stack of spinning doughnuts. Radial velocities can potentially redistribute the insoluble particles in the sample so that the slurry is no longer homogeneous in its properties across the annular gap. Solids can accumulate in lower velocity regions such as in rings along the beaker and bob walls. Vertical velocities could also accelerate settling of solids into the region below the annular gap.

Figure 18 shows a flow curve for SB2/SB3 SME product from run \#5 that was diluted to lower the yield stress to the lower end of the nominal DWPF SME product rheology window of 2.5 Pa yield stress and 10 cP consistency. This sample showed considerable time dependence as measured by differences between its up ramp and down ramp flow curve segments.

\section{Figure 18. Impact of Taylor Vortices on Time-dependence of SB2-3 SME Product}

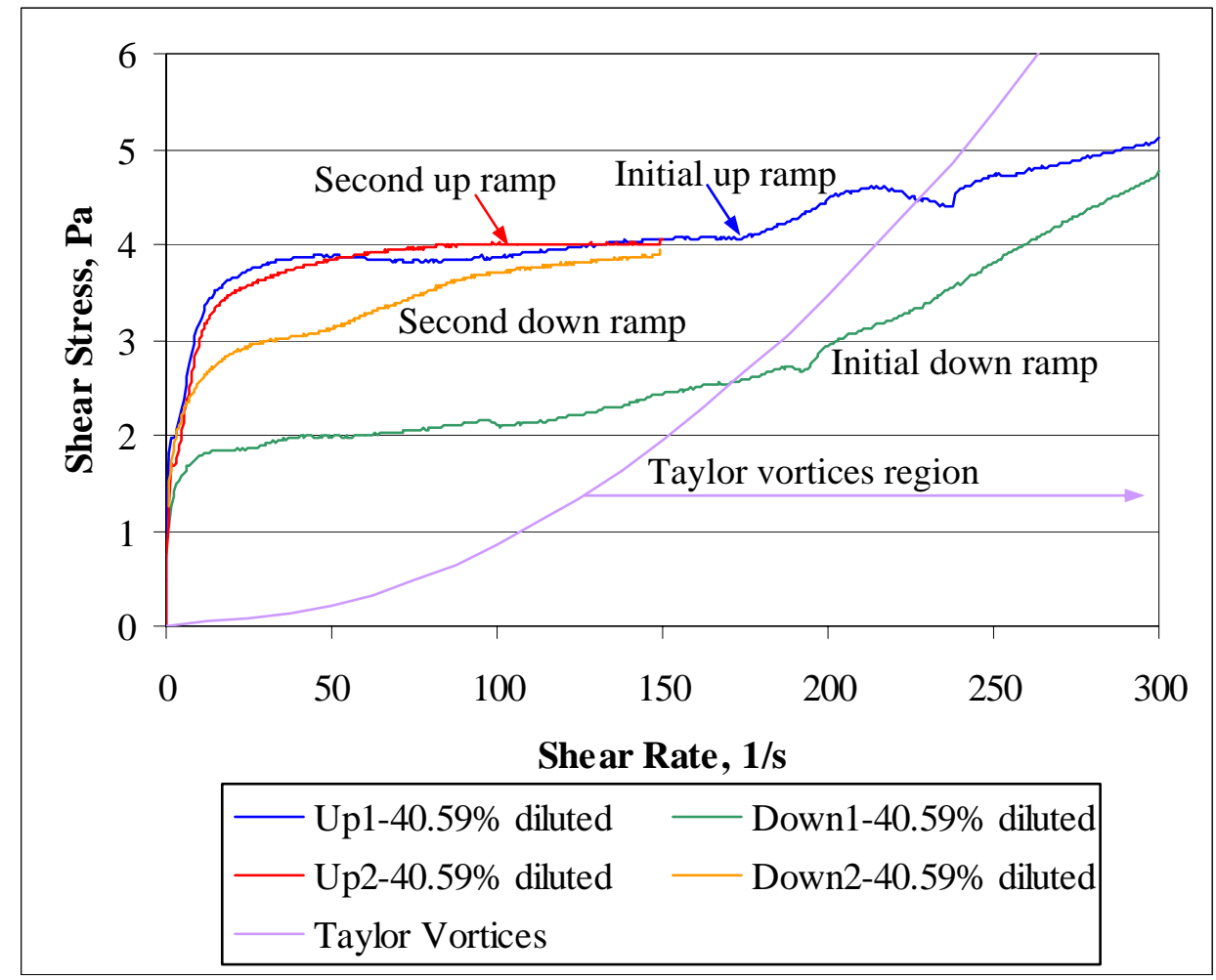

The ramp range was reduced for the second flow curve to $0-150 / \mathrm{s}$ instead of $0-300 / \mathrm{s}$. Testing was at a constant ramp rate, $1 / \mathrm{s}$ per second, for both runs. The second flow curve took half as much time, because it had half as large a shear rate range. The two up ramps are nearly identical from 0-150/s as expected in this case. The thixotropy implied by the area between the up and down curves, e.g. equation [4], was greatly diminished by avoiding the region of Taylor vortices and/or by halving the time in the rheometer. This dilution of the slurry was sufficiently thin that keeping the solids suspended for 10-15 minutes was an issue. This prevented the application of the multiple flow curve method to this sample.

Figure 19 gives data for a SB2/3 SRAT product. This sample was run with the Z38 bob instead of the Z41 bob. The Z38 bob was used because of sand and coal in the slurry that gave rise to stress spikes when using the $\mathrm{Z} 41 \mathrm{bob}$ with its narrower gap. Temperature was $25^{\circ} \mathrm{C}$. 
WSRC-TR-2004-00334

Revision 0

Figure 19. SB2/3 CETL SRAT Product Rheogram and Impact of Taylor Vortices, Z38

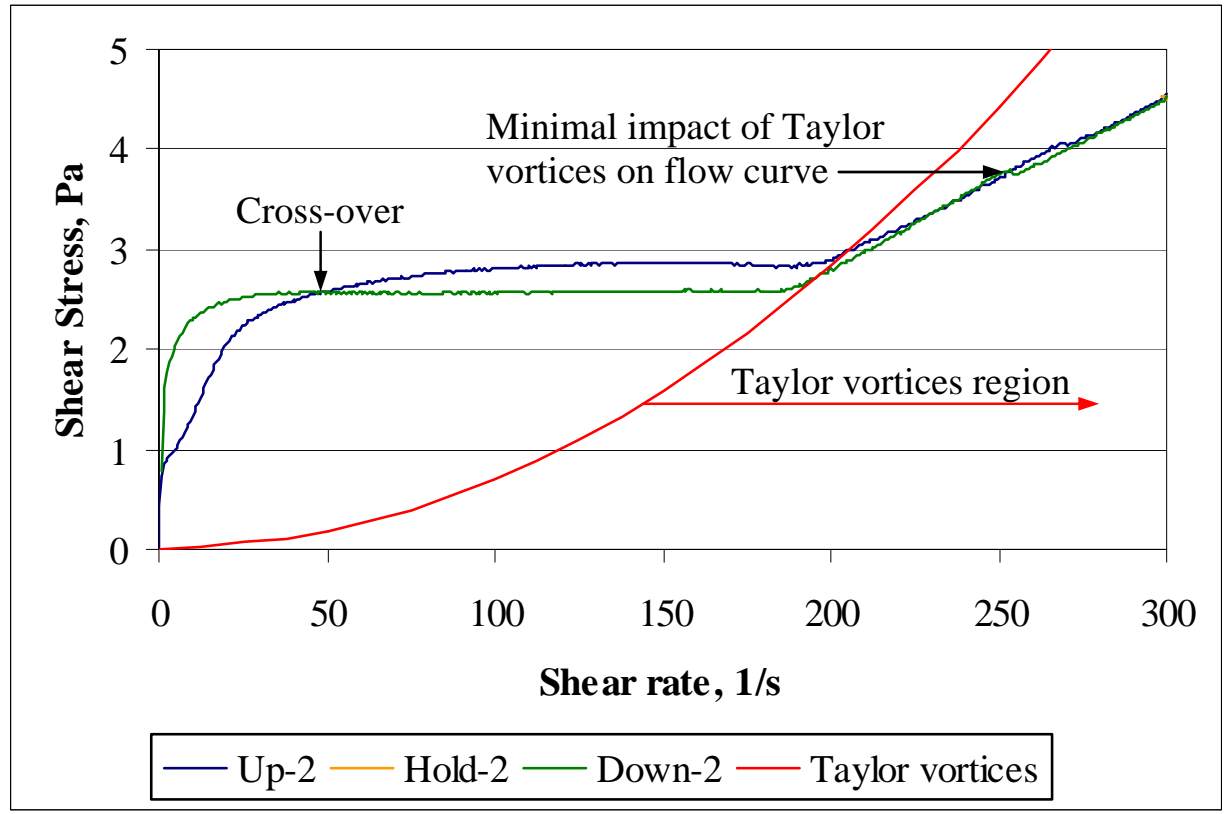

The slurry was fairly thin and had cross-over at a shear rate of about 45/s. Taylor vortices appeared when predicted by theory, red curve. The up and down ramps in the high shear rate range (right of the Taylor vortices trace) were nearly identical unlike the behavior of the SME product in Figure 18. This suggests that the homogeneity of the SRAT product slurry was not greatly altered by the Taylor vortices. This leads to the conclusion that it is the frit particles, rather than the sludge particles, in the SME products are the ones that are most impacted by the secondary flows while in the region of Taylor vortices.

Figure 20 gives rheometric data for the SB2 CETL Sludge sample. This sludge flow curve was made using the Z38 bob instead of the Z41 bob to test the character of the hump in the up ramp that was being studied primarily with the Z41 bob. Temperature was $25^{\circ} \mathrm{C}$. 
Figure 20. Impact of Taylor Vortices on SB2 CETL Sludge Rheogram

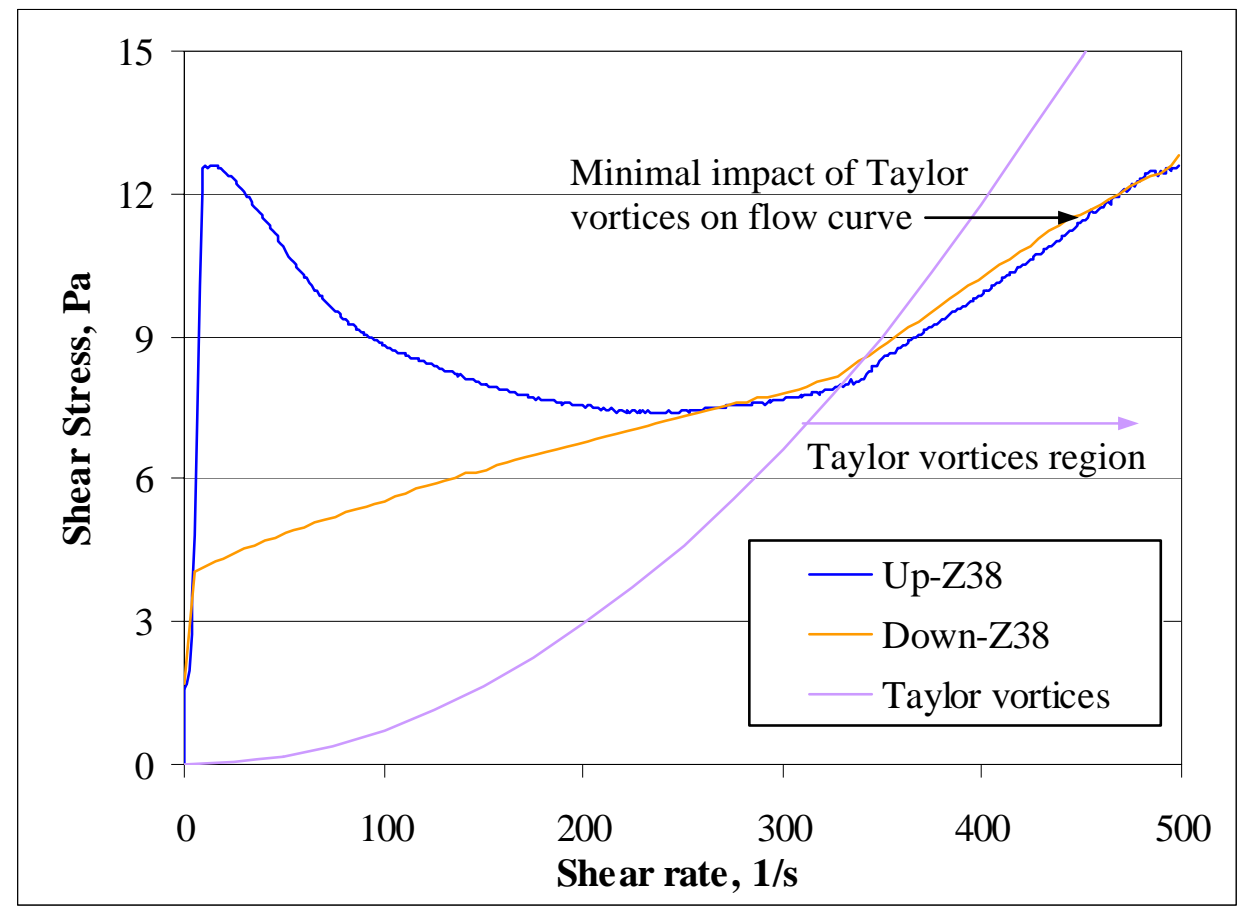

Use of the Z38 bob did not eliminate the hump in the initial up ramp. It was observed that the Taylor vortices appeared approximately when expected in spite of the hump in the up ramp. The sludge recovered on the down ramp along almost the same curve as the up ramp while in the region of Taylor vortices. This suggests that Taylor vortices did not have a large impact on the homogeneity of the sludge slurry. Combining the sludge and SRAT product data from Figure 19 and Figure 20 seems to indicate that typical waste slurry particles are not significantly segregated by a few minutes in the presence of Taylor vortices, or that any segregation does not significantly alter the rheological properties. This did not appear to be the case for the SME product containing frit in Figure 18.

Figure 21 shows another SME product from the SB2/3 acid window study that received further investigation during the method development work. The sample was run using the $\mathrm{Z38}$ bob at $25^{\circ} \mathrm{C}$. 
Figure 21. SB2/3 SME Product (140\% Stoichiometry), Z38

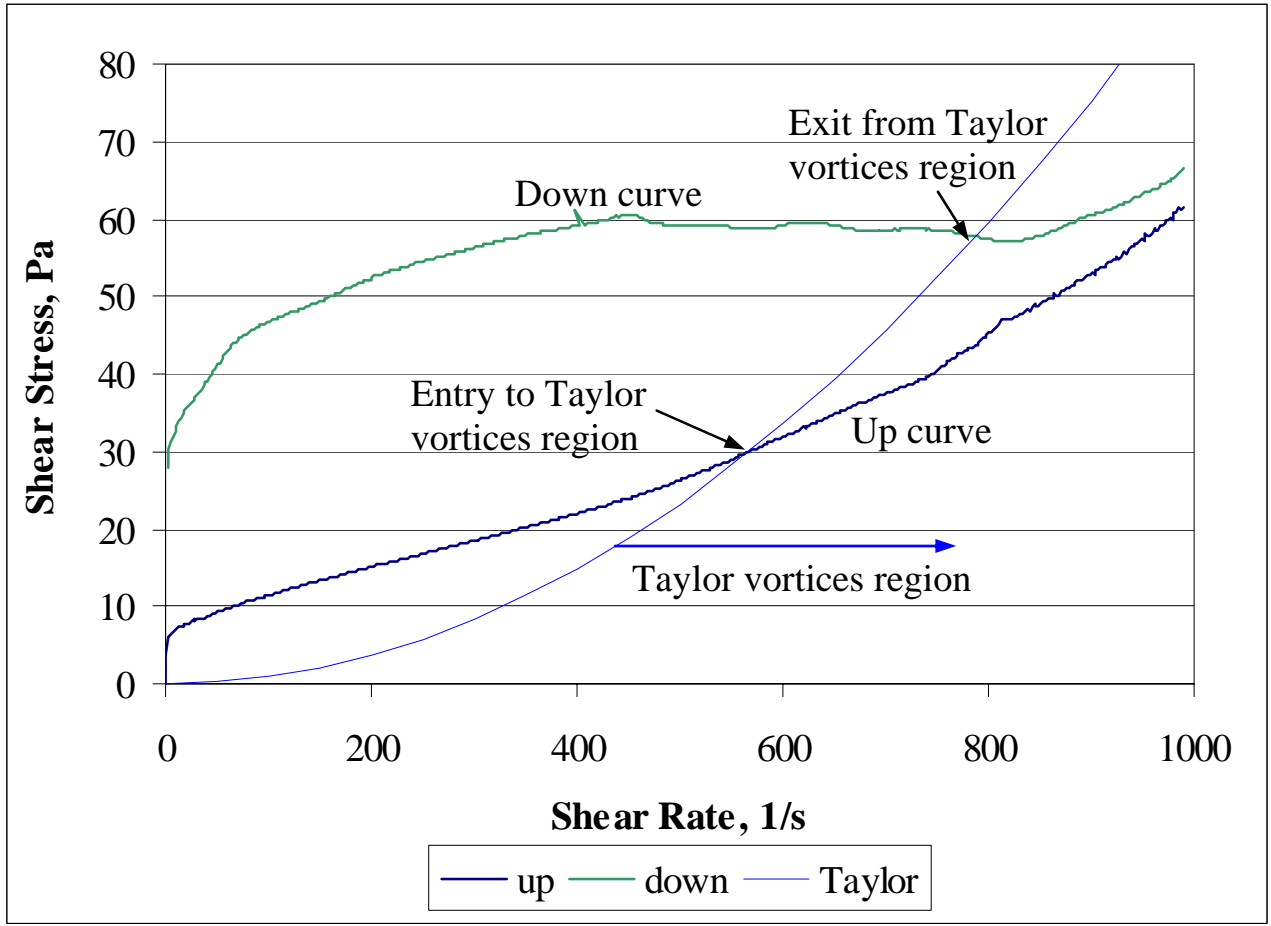

The sample did not reemerge from the region of Taylor vortices remotely close to where it entered. This suggests that the sample may have segregated significantly while the Taylor vortices were present (about four minutes).

Figure 22 shows data from one of the other SME products from the SB2/3 acid window study. This sample was also run using the $\mathrm{Z} 38$ bob at $25^{\circ} \mathrm{C}$. 
Figure 22. SB2/3 SME Product (185\% Stoichiometry), Z38

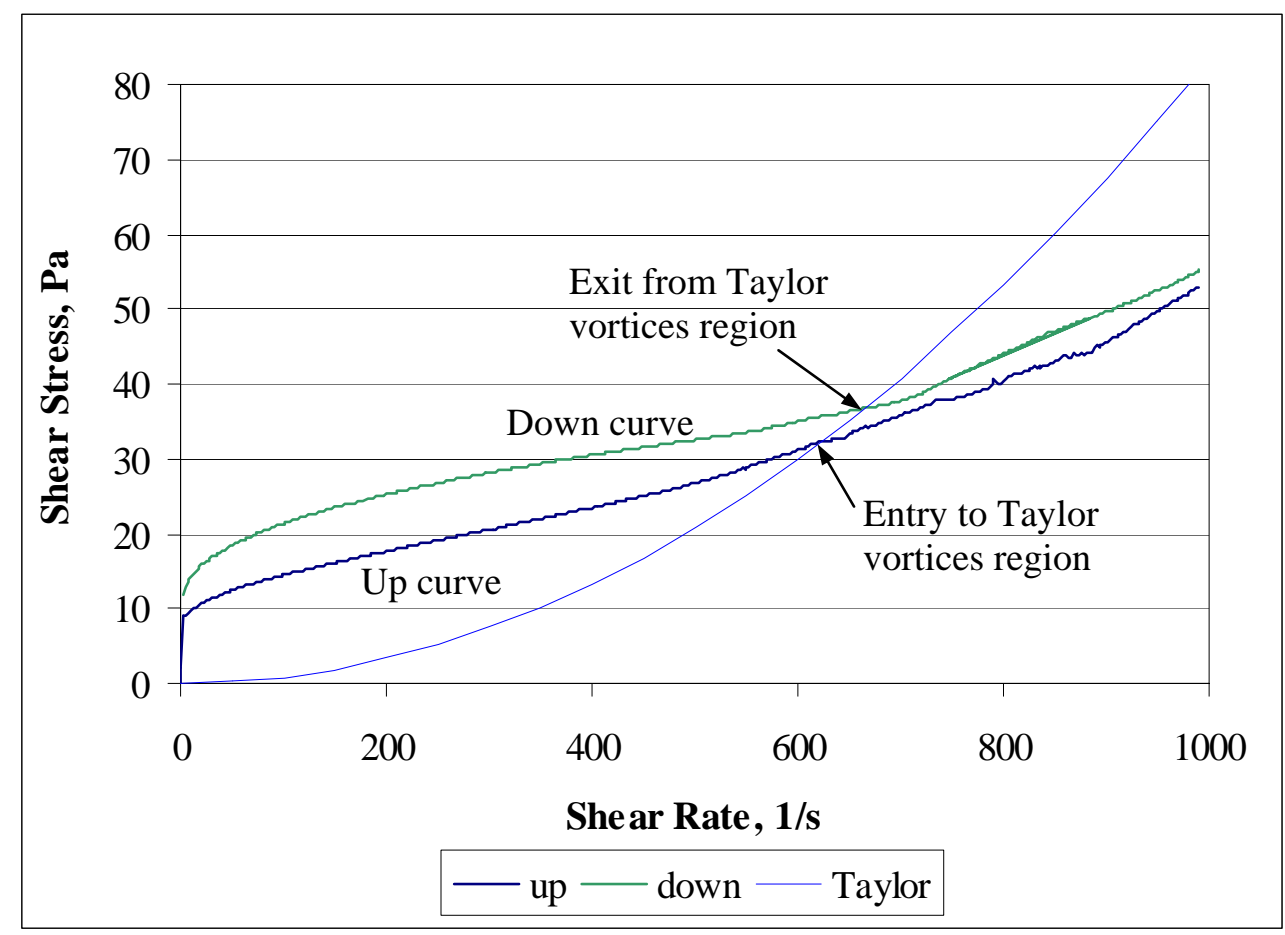

This only slightly thicker sample was much less impacted by Taylor vortices than the sample in Figure 21.

The behavior seen in the SME product samples is consistent with the sample partitioning into regions that are more solid-rich and regions that are lower in solids. The rates of increase in yield stress and consistency as a function of insoluble solids concentration are nearly exponential (two unique functionalities). Therefore, the minimum yield stress and consistency are measured when the sample is perfectly homogeneous. As the solids partition, the increased viscosity of the solid rich regions is not offset by the reduced viscosity of the solid lean regions which gives rise to a net increase in the apparent viscosity. These SME products all exhibited increased apparent viscosity in the down ramp following the time at high shear rate relative to the up ramp to some degree. The conclusion is that SME product slurries should not be sheared into the region of Taylor vortices. It also suggests that smaller instances of apparent rheopectic behavior may instead be due to frit migration in the annular gap.

\subsubsection{Assessment}

The controlled shear rate flow curve is the primary measurement used because it gives information about the behavior of the sample over a range of shear rates and over a period of time. This serves as an excellent preliminary characterization measurement. The data from this test often indicate that no further characterization is necessary.

The method, however, is primarily used to generate the Bingham plastic model parameters, yield stress and consistency. This model is suitable only for time independent rheological behavior. When time dependent behavior is observed, alternative approaches are required. When the samples are simulants, then one of the first questions should be "Is this a suitable rheological simulant for the radioactive slurry?”, i.e. does the radioactive material also have time dependent rheological properties? 
The controlled shear rate measurement benefits mathematically from using the smallest annular gap that is feasible. The minimum feasible gap per Alderman and Heywood (2004a, p. 30) would be a gap that is 30-50 times the mean particle diameter in the slurry. The narrow gap Z41 and MV1 bobs are suitable for sludge and SRAT product in the absence of large particle impurities, such as sand and coal, e.g. a $1 \mathrm{~mm}$ gap is 50 times larger than a 20 micron mean particle size sludge. The larger gap Z38 and MV2 bobs would be excessive for sludge and SRAT products most of the time. These bobs are perhaps too narrow for SME products depending on the method used to calculate the mean particle diameter of a bimodal distribution and on the waste loading (less frit, smaller mean particle diameter). The Z41 and MV1 bobs do not meet the 30-50 times the mean particle diameter criteria for SME products. The Z38 and MV2 bobs are about 2.5 times closer to providing 30 times the mean particle diameter than Z41 and MV1. Exactly how close they come depends on the choice of mean particle size (number or volume mean) and on the waste loading (higher waste loading, lower mean particle size).

These types of guidelines generally relate to the applicability of continuum mechanics models to the annular gap region. The slurry is heterogeneous in nature because it consists of distinct solid and liquid phases. If the slurry is divided finely enough, then it will either behave like pure supernate or like a solid particle, rather than like a slurry. The scale of the gap must be chosen such that it is much larger than the scale of the slurry structures before a slurry can be treated as a pseudo-homogeneous fluid. The Z31 and MV3 bobs provide even larger gaps than the Z38 and MV2 bobs. They would be better yet from the standpoint of creating a continuum fluid in the gap. Unfortunately, the amount of variation in the shear rate experienced by the sample across the annular gap is also increasing with increasing gap spacing. This creates another problem, that of matching a single shear rate to the measured shear stress when the bob is rotating at a fixed speed. In homogeneous fluids, this is the only consideration, and it drives the bob choice towards as narrow of a gap as is feasible. SME product slurries require a compromise between these two competing concerns. The Z38 and MV2 bobs seem to be the best compromise.

Testing with different shear rate ranges and ramp rates indicates that some samples are more sensitive to the choice of these parameters than others. It is recommended that the ranges and ramp rates be standardized. Samples that were run into the region of Taylor vortices appeared to be adversely impacted, at least in the case of SME products. Avoiding the region of Taylor vortices with all likely DWPF SME products would restrict the range of shear rates studied to about 0-200/s. Few slurries are actually studied at the thin end of the DWPF rheology window. Therefore, a nominal shear rate range of $0-300 / \mathrm{s}$ is recommended for SME products measured with the Z38 bob. This range is also suitable for MV2 bobs with the Shielded Cells and radiohood rheometers. The sludge and SRAT products appeared to be less susceptible. The recommended range for these could be 0-600/s. This range is larger than much of the older data taken at 0-350/s to 0-400/s ranges, but smaller than some of the more recent data taken with ranges from $0 / \mathrm{s}$ to $\sim 1000 / \mathrm{s}$. The recommended range is intermediate, and it is intended to keep future data as comparable to past data as possible, while simultaneously standardizing the measurement range.

The ramp times of five minutes have been fairly common in the past. These times were found to be satisfactory. Some samples can be measured more quickly without changing the results, but time dependent samples are poorly characterized by rapid ramps. The space between the up and down ramps shrinks as the ramp times are decreased for time dependent samples. Therefore, the ramp times should be kept at five minutes for sludge, SRAT product, and SME product slurries.

There is value in consistency of ramp rate and hold time among various data sets. For this reason the actual measurement protocol below recommends five minutes ramp segments with a one minute hold segment. Historically, this has been adequate to identify time dependence. Table 3 summarizes the recommendations for flow curve measurements. 
WSRC-TR-2004-00334

Revision 0

Table 3. Summary of Recommended Flow Curve Inputs

\begin{tabular}{|l|c|c|c|c|c|}
\hline Sample Type & $\begin{array}{c}\text { Bob } \\
\text { (Cylinder) }\end{array}$ & $\begin{array}{c}\text { Up/Down } \\
\text { Ramp Segment } \\
\text { Shear Rate } \\
\text { Range }\end{array}$ & $\begin{array}{c}\text { Up/Down } \\
\text { Ramp } \\
\text { Segment } \\
\text { Times }\end{array}$ & $\begin{array}{c}\text { Hold time at } \\
\text { maximum } \\
\text { Shear Rate }\end{array}$ & $\begin{array}{c}\text { Number of Data } \\
\text { Points per } \\
\text { segment: } \\
\text { up-hold-down }\end{array}$ \\
\hline Sludge & Z41 or MV1 & $0-600 / s$ & $300 \mathrm{~s}$ & $60 \mathrm{~s}$ & $300-60-300$ \\
\hline SRAT Product & Z41 or MV1 & $0-600 / \mathrm{s}$ & $300 \mathrm{~s}$ & $60 \mathrm{~s}$ & $300-60-300$ \\
\hline SME Product & Z38 or MV2 & $0-300 / \mathrm{s}$ & $300 \mathrm{~s}$ & $60 \mathrm{~s}$ & $300-60-300$ \\
\hline
\end{tabular}

When the researcher detects significant time dependence, it would be prudent to run a sample through two or more flow curves (multiple up and down ramps) to see if a rheological steady state flow curve can be obtained. It is recommended that larger volumes of sample be requested than are necessary to support 2-3 routine flow curve measurements in case additional sample is needed to characterize time dependence. The routine flow curve measurements can be made using the recommended shear rate ranges and ramp times as in the past.

Unusually thick SME product samples have been generated in the past two years. These samples have yield stresses in excess of 300 dynes $/ \mathrm{cm}^{2}$. There are additional problems associated with these samples compared to more normal SME products. One of these is getting the sample into the annular gap. The shear-free air pocket on the underside of the bob is often lost when loading these samples. This increases the area for shear to include both the sides and the bottom of the bob. This leads to somewhat higher torque load which leads to an elevated shear stress profile versus shear rate compared to if the air pocket is maintained. This would be a factor if very precise comparisons are necessary between these highly viscous SME products. In that case, it is recommended that bobs without the space for the air pocket be purchased and used. This would eliminate variability due to the amount of the air space that is replaced by slurry during a measurement. With the alternate bobs, the entire bottom surface would be wetted by slurry in every measurement, which would eliminate that source of variability in the data.

Another problem was encountered with thick SME products. These slurries had rheological properties that were very sensitive to the solids content, i.e. very sensitive to the water content. Small evaporation losses were noticeable in the rheological data on repeated measurements. This was found when working with $\sim 150 \mathrm{~mL}$ aliquots of larger ( 2L) volumes of SME product.

One final issue was noted with respect to SRAT and SME products. The SRAT and SME product slurries were found to not be in chemical equilibrium. There was an on-going process of dissolution and/or crystallization as these slurries sat on the shelf that was changing the rheological properties. This indicates that it is important to control the time between the end of the SRAT and/or SME cycle and the time that the rheological measurement is made. Alternatively, the samples can be left to sit for several weeks before any of the rheological measurements are made. More detail can be found in the companion report, Koopman (2005). 


\subsection{Controlled Shear Stress Flow Curves}

This is an alternative method for obtaining shear stress-shear rate data. It is similar to the controlled shear rate flow curve, section 3.1, in some ways. The main difference is that the shear stress (torque) is controlled and ramped instead of the shear rate (rotational speed). This creates a complication when the controlled shear rate flow curve has not been measured. The maximum shear stress (ramp end point) is a user input in the controlled stress flow curve measurement program rather than the maximum shear rate. The most straight-forward way to select the maximum shear stress is to take it off of a controlled shear rate flow curve at the stress corresponding to the maximum shear rate of interest. It can be assumed that a controlled shear rate flow curve will be available in most cases related to DWPF sludge, SRAT product and SME product samples, since it is the primary measurement method.

The shear stress can be ramped either linearly or logarithmically, as described in section 2.2.3. Virtually all data presented in this report were obtained using logarithmic ramping. This choice is not without issues, but is better than the linear ramp for characterizing the low stress region. There were issues early in the development phase that were confined to the low stress portion of the data. Switching from linear to logarithmic ramping put more of the ramp time and tabular data in the low shear stress region.

Tabular data were also taken using a logarithmic format. This format records data more frequently during the early portion of the ramp and less frequently in the later portion of the ramp. The two logarithmic styles in combination may have been excessive with respect to getting data in the low shear stress region. Issues with the quantity of tabular data can be worked by calling for the software to store data more frequently. Ramps contained 100 points initially. These became 300 point ramps by the end of the method development experiments, and could have just as easily been made into 1000 point ramps. No increase in ramp time is required to increase the number of recorded data points. Liquid-gel and thickthin slurry transitions in sample behavior occurred quickly. Sometimes fewer than ten data points in the set were relevant to a numerical analysis for a slope.

Several rheometric issues were investigated with the controlled shear stress flow curve. These were:

- What is a reasonable approach to making this measurement on a DWPF slurry sample?

- What does the controlled shear stress flow curve reveal about the true nature of the yield stress?

- How does the controlled shear stress flow curve compare to the controlled shear rate flow curve?

The answers to these questions are discussed in detail below. A brief overview is given to prepare the reader:

- First, to obtain accurate data about the true yield stress (defined as the transition from a solid or gel-like state to a fluid state) of DWPF simulant slurry samples required that the sample be held for a non-trivial length of time in the instrument before initiating the stress ramp. The final recommendation was to hold it for at least ten minutes. If rapid settling is an issue, then this method will not produce meaningful true yield stress data.

- Second, there was almost always a true yield stress detected in DWPF slurry samples, and it was always significantly less than the Bingham plastic yield stress. 
- Third, the data plotted in the form of a shear stress-shear rate flow curve can be virtually identical to that seen in the controlled shear rate flow curve. This occurred when the sample being studied had negligible time dependence. The two flow curves were not identical when the samples had significant time dependent behavior, such as the SB2 CETL sludge (hump) and some of the SME products.

- Even when the controlled rate and controlled stress flow curves were not identical, there were large-scale similarities in appearance that indicated that the measured properties were coming from the same material.

Some examples will be given below. Additional examples can be found in Koopman (2005) for some of the slurries that exhibited unusual rheological behavior.

\subsubsection{Controlled Stress Ramp of Viscosity Standard}

The first set of data, Figure 23, is a comparison of the linear ramp and logarithmic ramp applied to a Newtonian viscosity standard. Both sets of data are from the controlled stress ramp up. These ramps were both $300 \mathrm{~s}$ in duration. The linear ramp was from 0-20 Pa. The logarithmic ramp was from 0.01-4 Pa.

Figure 23. Controlled Stress Ramps of NIST Traceable N35 Viscosity Standard

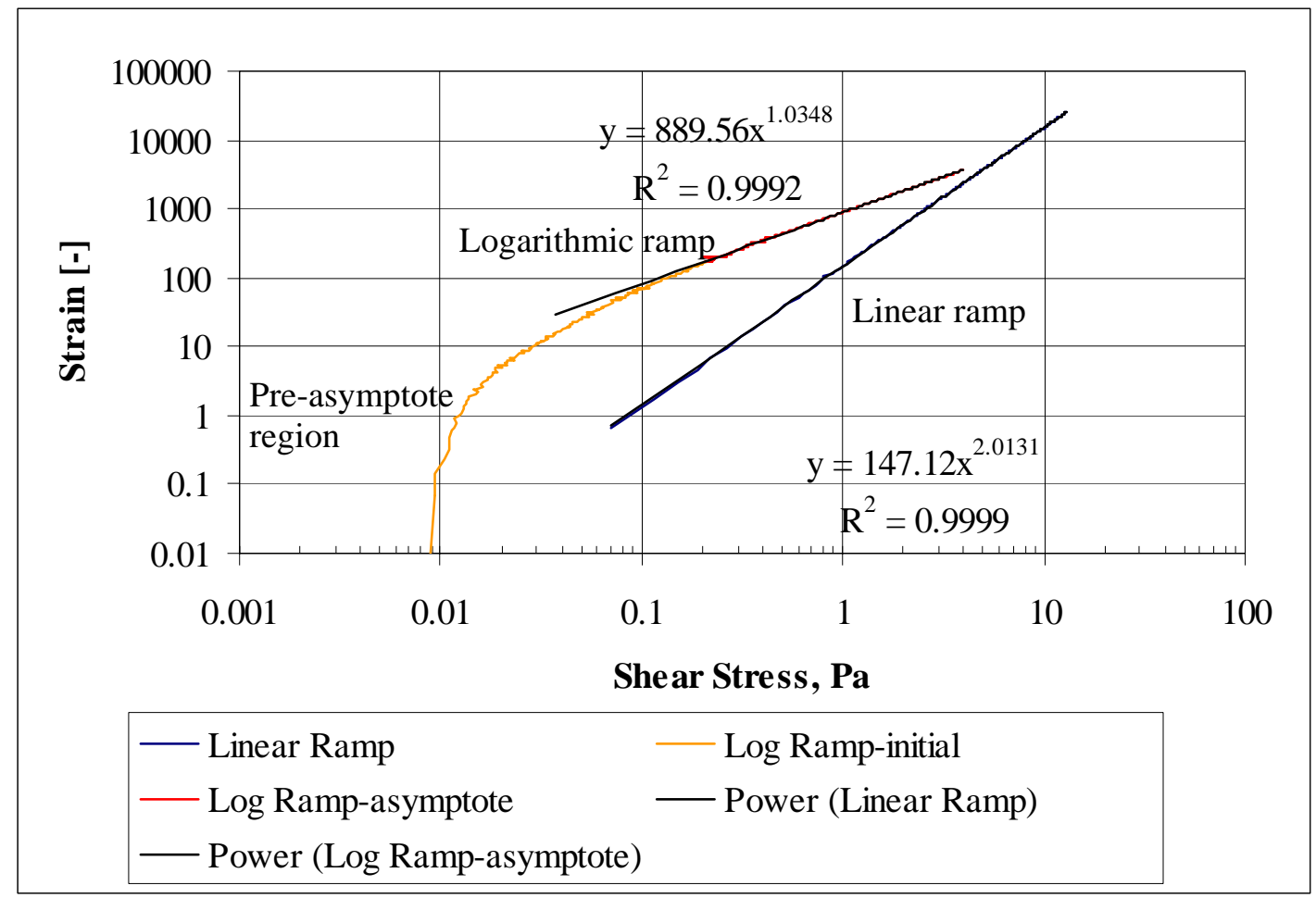

The linear stress ramp had slope 2.0131 versus an expected slope of 2.0 for a Newtonian liquid. Virtually all points were aligned with the power fit line. The logarithmic stress ramp had a slope asymptote of 1.0348 versus an expected asymptotic limit slope of 1.0 for a Newtonian liquid (see discussion in section 2.2.4). This was excellent agreement, and a validation of the mathematical analysis. The region from $0.01 \mathrm{~Pa}-0.1 \mathrm{~Pa}$ was not yet in the asymptotic limit for the logarithmic ramp. The onset of the asymptotic limit can be adjusted to lower stresses somewhat by starting at lower initial shear stresses per eqn. [15]. 
The measured viscosity in the linear ramp was about 49-50 cP, while the measured viscosity in the logarithmic ramp was 54-55 cP. The nominal viscosity of the standard was $51.2 \mathrm{cP}$. The variations are typical. (Another logarithmic ramp on a third day gave 50-51 cP, so there is no reason to conclude that the ramp method causes variations in the measured viscosity result.) Strain was fairly large in both sets of data. This is a consequence of the absence of a yield stress. Fluid deformation commenced as soon as shear stress was applied.

\subsubsection{Controlled Stress Ramps of Process Samples}

SB2 USC sludge simulant data are presented next. This was a well-behaved sludge with minimal time dependent behavior. Figure 24 gives data from four tests. The time that the sample was held in the rheometer before initiating the shear stress ramp was varied. The first three runs started at $0.05 \mathrm{~Pa}$, while the ten minute hold run started at $0.01 \mathrm{~Pa}$. Ramp time was $300 \mathrm{~s}$ in all four cases. Black circles are used to indicate the location of yield stress transitions.

Figure 24. Controlled Shear Stress Data for SB2 Sludge (USC)

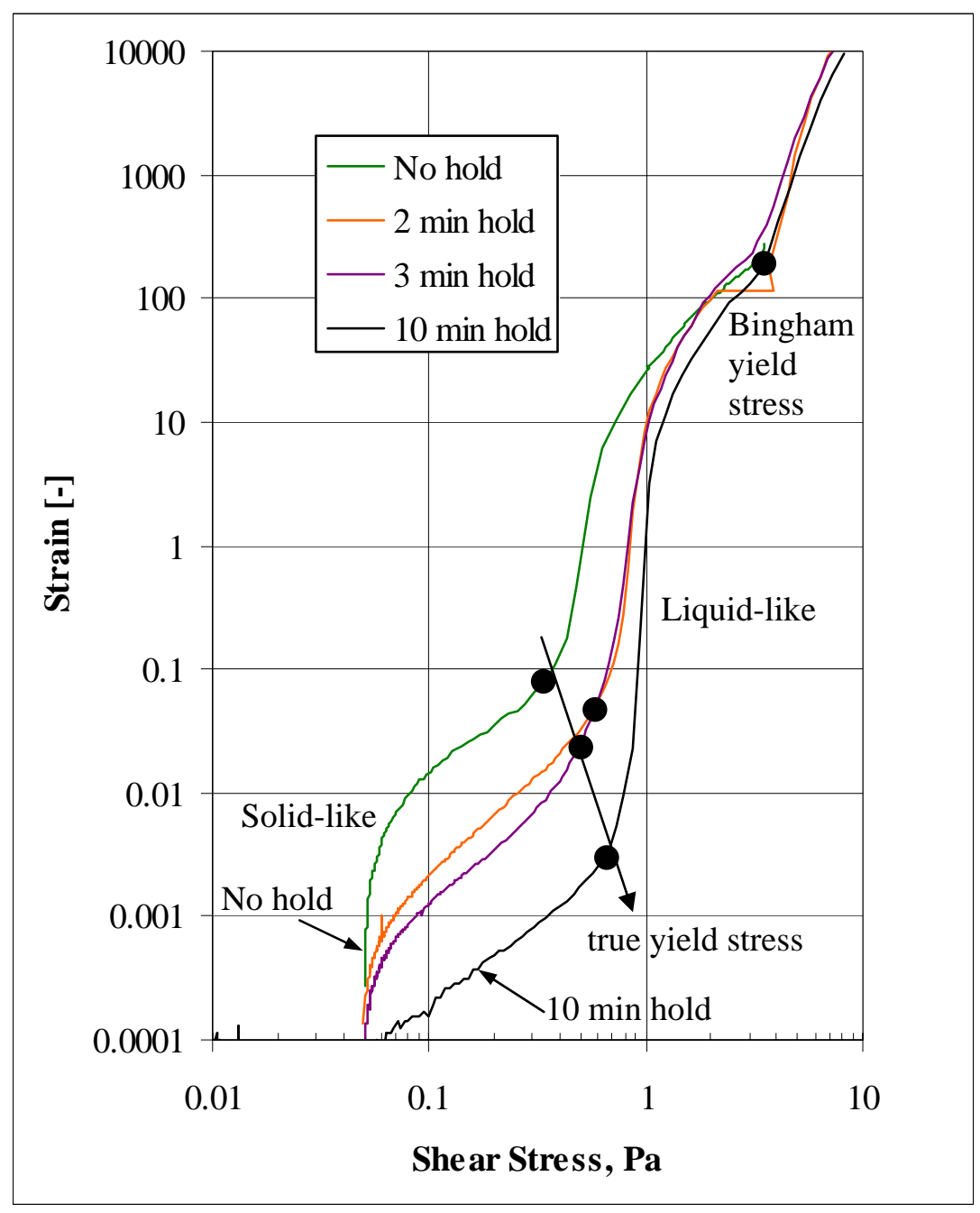

The four black circles below a strain of 0.1 correspond to the true yield stress of the slurry. That this was the case was more readily established with the oscillation tests than with the controlled stress ramp. 
Specifically, the data show four measurements of the true yield stress of the slurry at the time that the shear was applied. This gave four results. This is interpreted to mean that the slurry sample was building structure over time while it was held at rest in the rheometer, e. g. gelation. Separate measurements of the rate of solids settling indicated that it was not an issue at this time scale. As structure was developed, the yield stress increased and the rigidity increased.

The ultimate true yield stress, or yield stress after full gelation has occurred, was difficult to measure quickly. Mixing and loading the sample destroys most, if not all, of the gel structure that might be present. As the hold time in the instrument increased, the sample had more time to reform the colloidal structure and associated bonds that were destroyed in the process of driving the sample from the bottom of the beaker up into the annular gap. These data show the colloidal structure rebuilding per the arrow.

Significant findings:

- As the time before applying the shear stress increased, the amount of stress required to produce a given deformation (strain) also increased, i.e. the slurry became more rigid.

- As the time before applying the shear stress increased, the amount of stress required to drive a transition from solid-like to liquid-like behavior also increased, i.e. the measured value for the true yield stress increased. The method must allow time for the structure to build before initiating shear stress when obtaining the true yield stress is the goal.

- Slopes left of the arrow ranged from 1.26 to 1.62, which is a bit large for a true solid (exactly one expected), but are too low to be a fluid (greater than $1.99 .$. expected). Slopes after yielding ranged from 7-19. The slopes after yielding were very sensitive to the data range fitted, however they were clearly much greater than the slope of two expected for a Newtonian liquid. This indicates that the samples were now fluid. There were typically only 5-10 points in the sharp vertical region just past the true yield stress when taking 300 data point ramps. This is the reason for suggesting more data points per ramp and/or switching to a linear distribution.

- The fifth black circle at about 3.7 Pa marks a second transition that was relatively common to all four holding times. This is near the Bingham plastic yield stress from the controlled shear rate flow curve. This transition was not expected to depend on hold time based on historical data. That was confirmed by all of the controlled stress ramp data.

The technique above could be developed into a method for monitoring the rate that gel structure builds into a slurry at rest. A series of samples could be prepared. Each would be well mixed, and then introduced into the rheometer in a standardized manner. Each sample would be held for a different length of time before initiating the shear stress ramp. A plot would be constructed of the true yield stress versus hold time. This would provide a rate-based parameter that could be used to characterize different slurries. Such data would apply to slurries in pipes that have stopped and then restarted flow.

The recommended mathematical method for finding the true yield stress is illustrated below. Data from the SB2 USC Sludge simulant sample using the Z41 bob was selected to illustrate the method. 
Figure 25. Analytical Determination of True Yield Stress

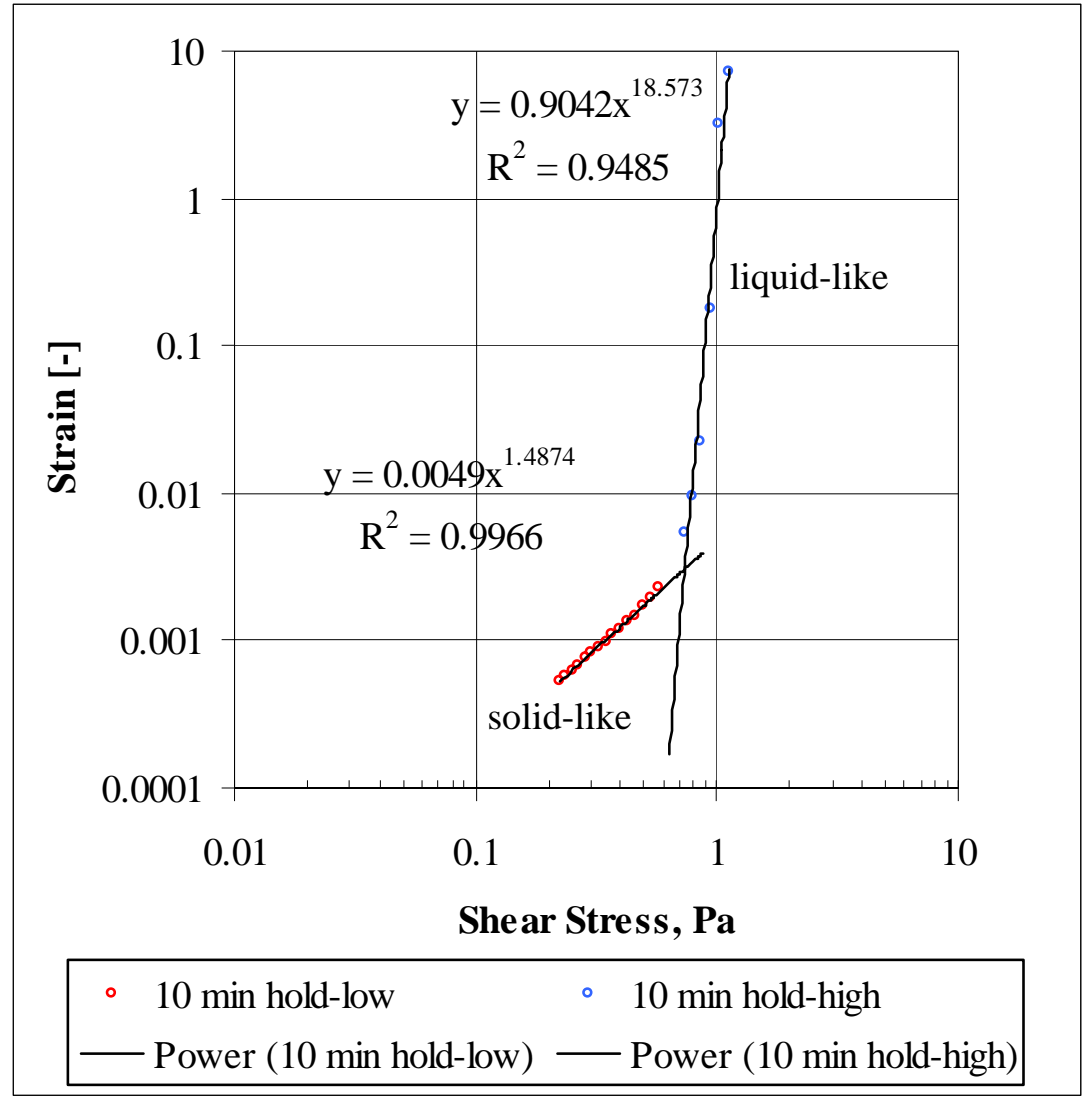

Solving the two equations on Figure 25 simultaneously gives an estimate of the true yield stress of 0.74 $\mathrm{Pa}$. The same technique can be applied to the data around the upper yield stress (Bingham plastic yield stress). It is often quicker to draw the lines on a graph and read the shear stress of the intersection than it is to fit and solve the two power equations.

Figure 26 compares the data from the 2 minute hold and 10 minute hold controlled stress ramps to data obtained during a controlled shear rate loop using the traditional linear flow curve axes. Data are for the SB2 USC Sludge simulant sample taken using the Z41 bob. 
WSRC-TR-2004-00334

Revision 0

Figure 26. Controlled Stress Compared to Controlled Rate Flow Curve

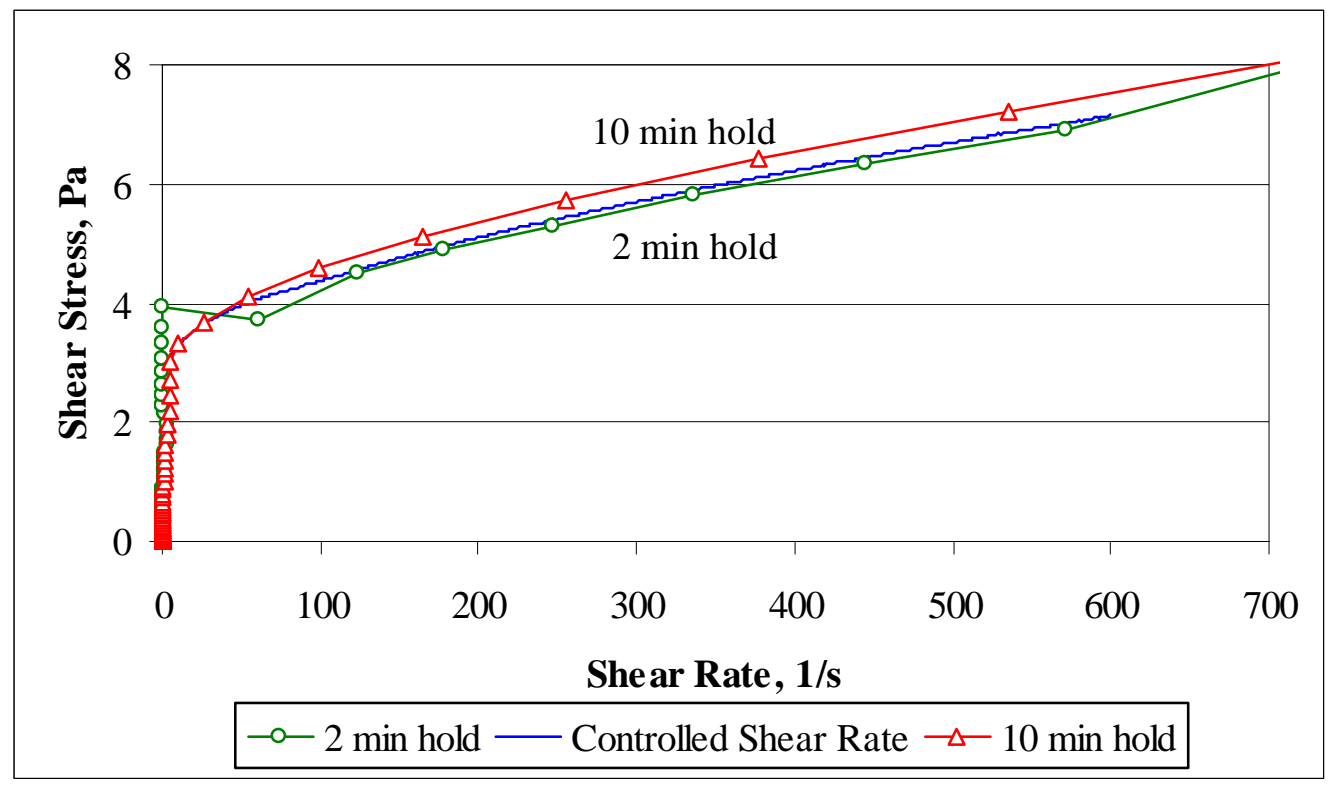

The two controlled shear stress flow curves bracket the controlled shear rate data. Data fall within normal limits of reproducibility for controlled shear rate flow curves. Fitting the shear rate from 100/s to 600/s gives a Bingham plastic yield stress slightly under $4 \mathrm{~Pa}$, which is comparable to that obtained by paired power law fits to the upper transition on the log strain-log stress graph in Figure 25. Note, however, in Figure 26 how few data points are on the controlled stress ramps above a shear rate of 10/s. This is a consequence of the logarithmic ramp coupled with the logarithmic distribution of recording times for the data. This could be overcome by allowing one or the other to revert to linear mode, or by taking more total data points. The time from $2 / \mathrm{s}$ to $535 / \mathrm{s}$ was 68 seconds, not the $5-10$ seconds that might be guessed from the number of points. Nevertheless, this compares to about 266 seconds in the linear shear rate ramp. This ramp rate effect was found to be a source of more significant differences between the controlled rate and controlled stress flow curves for samples that had more time dependent behavior. The effect was similar to that of reducing the ramping time in section 3.1.

Small samples of four SB2/3 CETL SME products were available from the acid stoichiometry study, Baich (2004a). These were found to have increased in wt. \% total solids in the four months between when they were made and when they were used in this study. Nevertheless, a series of controlled stress measurements was made on them using the Z38 bob. 
Figure 27. Controlled Stress Ramp on Acid Stoichiometry Study SME Products

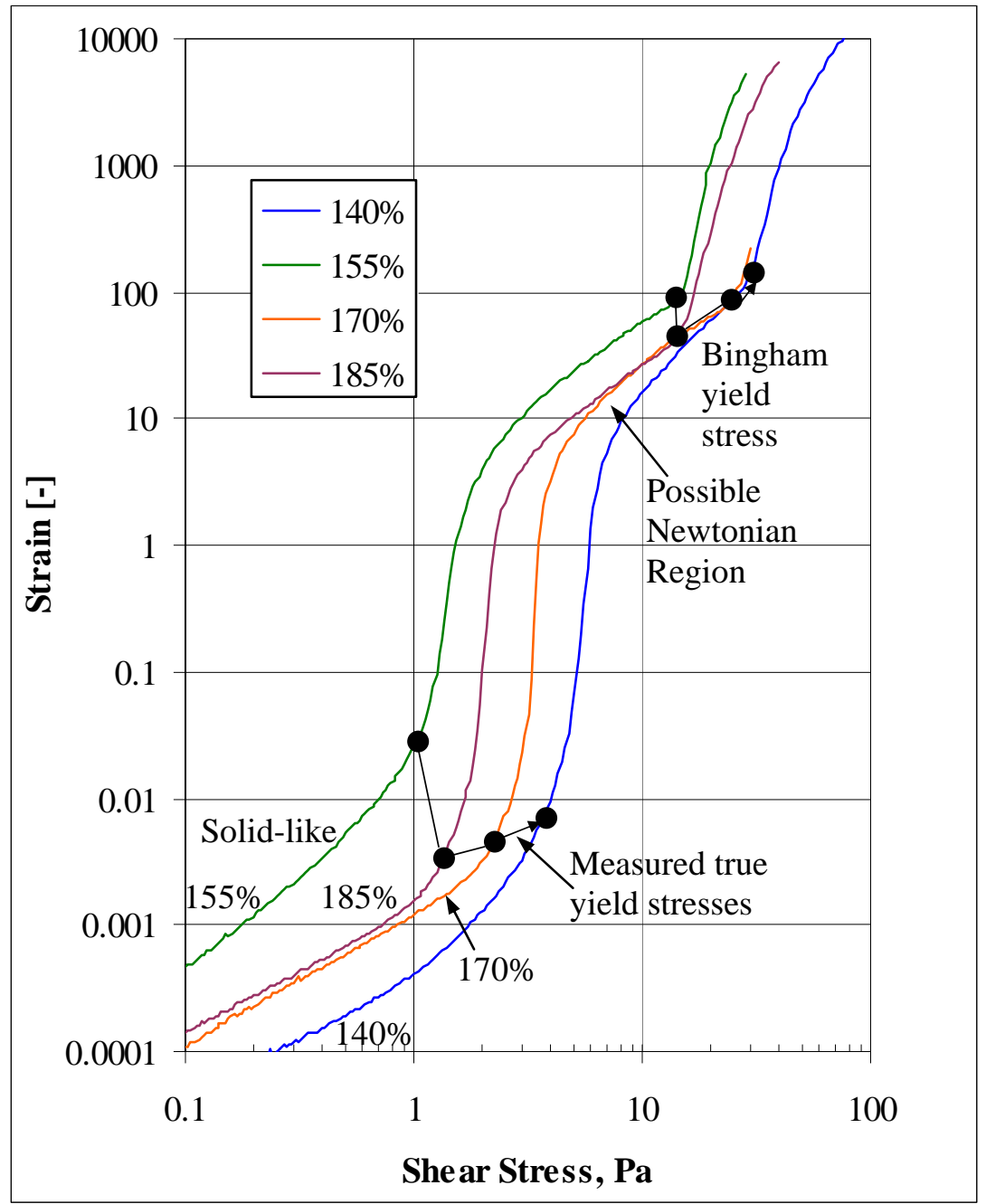

Eight yield transitions are marked with black circles, two for each sample. All four samples exhibited a true yield stress between 10 and 45 dynes $/ \mathrm{cm}^{2}$ at strains less than 0.1, and a Bingham plastic yield stress greater than 100 gynes $/ \mathrm{cm}^{2}$ at strains greater than 10 . This was a general pattern seen for all but two samples in the experimental study. All four samples above were held for ten minutes before applying stress.

It is noteworthy that the relative ranking of the four yield stresses was the same for both the true yield stress and for the Bingham plastic yield stress. Prior to this measurement, it was not known whether the true yield stresses could be expected to rank the same as the Bingham plastic yield stresses. While one study does not prove that this will always be the case, it does set a baseline against which future data can be evaluated.

There was sufficient sample to run two of \% acid SME products twice. Slopes prior to the true yield stress ranged from just over 1.0 to just under 2.0 in the six data sets. An unexpectedly similar result was obtained for the slopes just prior to the Bingham plastic yield stress. This, and similar data from various other samples in the study, suggests that a subset of the samples in this study might be exhibiting either a Newtonian fluid regime or re-establishing a structured or ordered state at shear stress levels between the 
two yield stresses. This was unexpected, and the asymptotic behavior of the logarithmic ramp precludes distinguishing based on the slope alone. The other evidence can be taken either way. It is hard to argue that structure survived at deformations from the starting point of ten gap spacings. On the other side, several samples nearly stopped the bob in this region (slope of near zero), suggesting that they had somehow gained rigidity. There is only a very small probability that this was caused by an unusually large particle. This phenomenon was difficult to reproduce on any given sample. Rheological literature would suggest that the Newtonian fluid region is the favored explanation. Many non-Newtonian fluids have a Newtonian limit at low shear rates. This includes slurries and polymer-based samples.

The relative ranking of the rheological properties for the four acid stoichiometries is not the same as that given in Baich (2004a). The four samples suffered varying degrees of drying in the time between preparation and this study. SME products also appear to not be in solid-liquid equilibrium until some time passes. The SME samples that had dried more, thickened more. Those that had dried less, thickened less. These samples are in the rheological region where small variations in total solids can produce significant variations in the Bingham plastic yield stress. In a rheological sense, the $140 \%$ sample was three times thicker, the $155 \%$ sample was about $25 \%$ thicker, the $170 \%$ sample was about twice as thick, and the 185\% sample was about 50\% thicker than in December, 2003. The wt. \% total solids (TS) changes that led to this are summarized in Table 4.

Table 4. Changes in Wt. \% Total Solids of SB2/3 SME Products

\begin{tabular}{|l|c|c|}
\hline Sample & Dec. 2003 & April 2004 \\
\hline $140 \%$ Acid SB2/3 SME & $50.4 \%$ TS & $55.5 \%$ TS \\
\hline $155 \%$ Acid SB2/3 SME & $50.6 \%$ TS & $52.4 \%$ TS \\
\hline $170 \%$ Acid SB2/3 SME & $49.9 \%$ TS & $53.6 \%$ TS \\
\hline $185 \%$ Acid SB2/3 SME & $49.8 \%$ TS & $53.3 \%$ TS \\
\hline
\end{tabular}

The yield stresses from the controlled stress tests track the total solids, $140 \%>170 \%>185 \%>155 \%$. Other factors, such as the wt. \% insoluble solids, $\mathrm{pH}$, and particle size, are also known to be factors.

Additional SME product was obtained from the SB2/3 processing variability study, Baich (2004b). The SME product obtained was from Run 5 (the heel simulation). No SME product data had been obtained near the lower limits of the DWPF design basis, since most available SME products were near or above the upper limits of the DWPF design basis. This SME product was diluted with de-ionized water to produce three samples with lower total solids content, and also lower yield stresses. Dilution 2, 40.59\% diluted, was very close to the lower limits of the DWPF design basis for the SME. Figure 28 gives the strain-stress data. 
Figure 28. Non-typical strain-stress plot of SB2/3 CETL SME product.

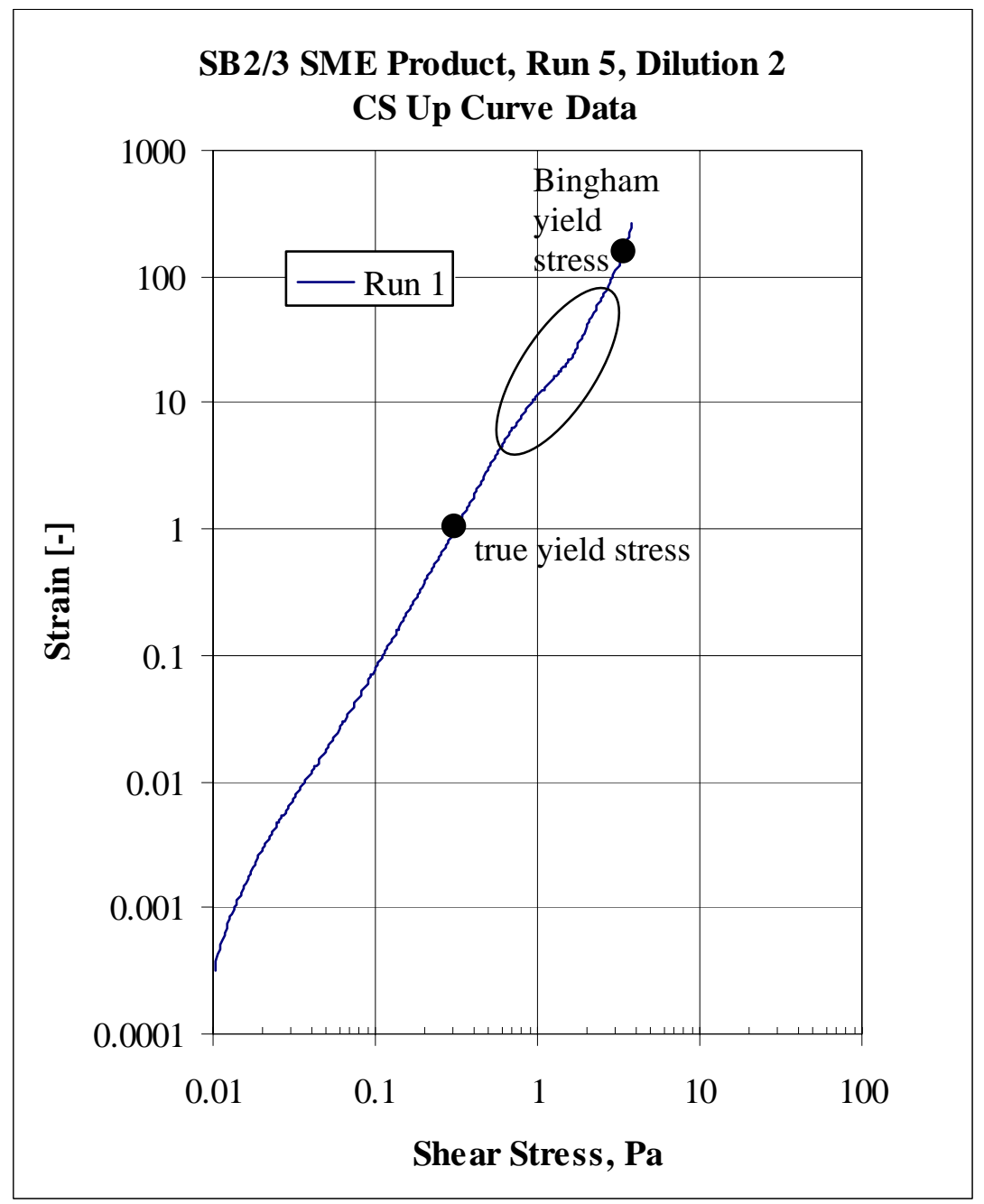

The controlled stress ramp data do not give either the true of the Bingham yield stresses from the strainstress plot. As the slurry was thinned, the S-curves seen in most of the other testing flattened into a nearly straight line. The true yield stress, as given by the oscillation stress sweep technique, section 3.5.1, lies in the neighborhood of $0.3 \mathrm{~Pa}$. Slopes are approximately two both before and after this point. The strain at this stress is unusually large for it to be a yield point. A yield strain less than 0.2 was seen with most samples. This sample was held for ten minutes before applying stress. It may be that it had been thinned to a point where very little structure could form. The Bingham plastic yield stress is approximately 3.5 $\mathrm{Pa}$.

The shape of the data between the two yield stresses (enclosed by oval in Figure 28) did have some slight similarity to that seen with the other samples, but the slope changes were clearly more muted. Figure 23 indicates that the stress is probably large enough at $0.1 \mathrm{~Pa}$ for the Dilution $2 \mathrm{SB} 2 / 3 \mathrm{SME}$ product sample to be exhibiting either solid-like or Newtonian fluid-like behavior (both have slope one), and this was not indicated by the data. This illustrates one of the possible anomalies that can be obtained by this method. The oscillation stress sweep data on this sample were much clearer in delineating the region of the true yield stress. This emphasizes the need for a complimentary set of measurement techniques that can remove ambiguity from individual measurements. 
SB2/3 CETL SRAT product exhibited the same behavior as Dilution 2 of the SB2/3 CETL SME product from Run 5 above (no clear true yield stress). The oscillation stress sweep gave an upper limit of $0.03 \mathrm{~Pa}$ for the true yield stress of this sample. This sample was analyzed using the Z38 bob because of the small quantities of sand and coal added to the sludge. The Bingham plastic yield stress was about 2.5 Pa. The implied difference between the two yield stresses in this case was of order 100. The totality of the test data did not indicate that the ratios of true yield stress to Bingham plastic yield stress would necessarily fall within a narrow range. Consequently, the true yield stress cannot be estimated with any confidence by applying a factor to the Bingham plastic yield stress. These were the only two samples that had unusual strain-stress plots, and both were examples of thin slurries of SME product and SRAT product respectively.

The next sample is an example of data for a SRAT product. This is SRAT product prepared from SB2 CETL sludge simulant (hump). There was no hump in the SRAT product controlled shear rate data, so processing may have eliminated whatever caused the hump. This sample was considerably thinner than the above SB2/3 CETL SME product (Dilution 2). 
Figure 29. SB2 CETL SRAT Product at Two Hold Times, Z41

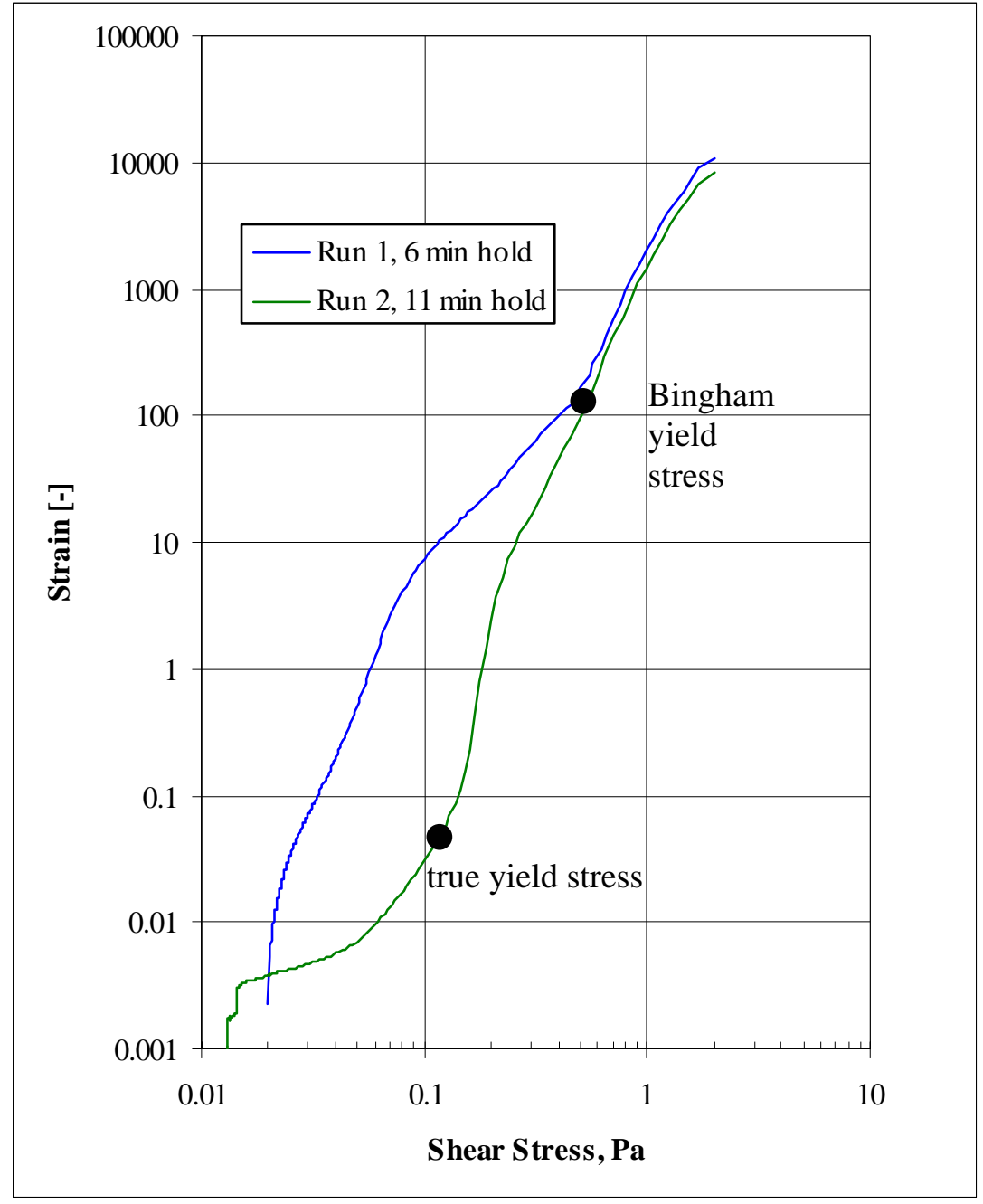

This illustrates more strongly what can happen to the true yield stress region when a sample is not held long enough before initiating stress on it. The black ball at $0.11 \mathrm{~Pa}$ marks the analytical true yield stress from the 11 minute hold curve analysis. The black ball at $0.5 \mathrm{~Pa}$ marks the extrapolation yield stress by the Bingham plastic model. (This is more obvious from a shear stress versus shear rate plot.) The two strain-stress data sets are fairly similar above the Bingham plastic yield stress. This was common, and it confirmed that the hold time was not critical to the free-flowing portion of the data that is of most interest.

The existence of a true yield stress is not even hinted at in the six minute hold data, while it is clear in the 11 minute hold data. These sets of hold time versus true yield stress data collectively showed the need for at least ten minutes of hold time before initiating shear stress on the sample. As much hold time as is practical would be best up to the point where gravity settling of the slurry particles corrupts the sample more than holding it stationary allows more gel-structure to reform. Two oscillation stress sweeps were made on this sample, one each with a 6 minute and an 11 minute hold. Both show a true yield stress transition in the region of 0.10-0.15 Pa.

Data on this thin SRAT product, as well as other samples, indicate that DWPF slurry samples should be assumed to have some non-zero true yield stress for all practical purposes. This yield stress existence 
issue had arisen in the past. ThermoHaake's technical representative thought that if SRNL explored the low stress region more carefully, then the data might indicate that there was no true yield stress. The existence of a true yield stress is important to sedimentation issues (settling of the solid particles in the absence of a bulk velocity). Gravitational forces on particles below some critical size are inadequate to overcome the yield stress force suspending the particle.

The true yield stress is probably more important than the Bingham plastic yield stress when analyzing the draining of films down the walls of process equipment. This relates to plant issues such as decontamination of equipment. If, in addition to a high yield stress, the film does not readily incorporate rinse water into the matrix, then the yield stress will be slow to change, and the film will be harder to remove. This could happen if the slurry is particularly cohesive. Also, the film thickness is expected to increase with increasing yield stress. Thicker slurries would be expected to be harder to rinse off, and this is confirmed in the laboratory when cleaning up the rheological equipment. Slurries that appear to be tacky in the DWPF Decon Cell, may simply be slurries with higher than normal true yield stresses, perhaps coupled with an increase in internal cohesiveness.

Some general conclusions can be drawn regarding the controlled shear stress ramp.

- First, most slurry samples showed two yield point transitions if the shear stress range was wide enough, and if the sample was held long enough to re-establish the gel-structures destroyed during sample loading.

- $\quad$ The true yield stress was generally associated with a strain below 0.1 and above 0.001 .

- The Bingham plastic yield stress was seen as a slope change on the strain-stress log-log plot in most cases, but was more readily obtained numerically by plotting the shear stress versus shear rate data in the same manner as used in the controlled shear rate flow curve analysis. The transition on the strain-stress plot typically came at strains greater than 10 .

- Ten minutes of holding was a minimal time to ensure a good chance of detecting the true yield stress. Consistency of hold times was important in comparing data from two or more samples.

- Combining a controlled shear stress ramp with the controlled shear rate hold and controlled shear rate down ramp elements from the traditional controlled shear rate flow curve would give a hybrid data set that contained a lot of rheological information.

The essential findings related to this technique were used to develop some recommended measurement protocols, Koopman (2004b).

\subsubsection{Assessment}

The controlled shear stress flow curve is a useful tool. It can confirm the results of the controlled shear rate flow curve measurement. The method produces different results for samples with unusual controlled rate flow curves, because the shear stress ramping is always either up or down and never includes updown combinations necessary to produce humps. Consequently, there is no likelihood of obtaining a hump or other structure where shear stress falls with increasing shear rate. This feature is expected to generate superior flow curves to the controlled shear rate mode in the sense that the data should be closer to the true steady state behavior of the sample initially.

The controlled shear stress flow curve measurement can be enhanced by adding a constant shear rate hold at the end of the ramp to test for thixotropy as well as by adding a down ramp that gives another flow 
curve. The down ramp can be either controlled stress or controlled shear rate. (Down ramp and hold rheometer job segments were not the focus of the controlled stress method development work.)

The controlled stress flow curve measurement can be made as rapidly as the controlled shear rate flow curve if information about the low stress behavior is not desired. This is roughly once per 16 minutes, Table 5, for cold samples with spare bobs (spares are needed so that the instrument can be running one sample while the bob and cup from the previous sample are being cleaned). The measurement can also be made as rapidly as the controlled shear rate flow curve with a ten minute hold before applying shear stress if there is no hold after the ramp and a rapid down ramp. Either method is competitive for resources. A controlled shear stress flow curve job structured to do everything would take about 24 minutes, Table 5. The job breakdown would be as follows:

Table 5. Structure of Controlled Shear Stress vs. Controlled Shear Rate Flow Curve

\begin{tabular}{|l|c|c|}
\hline Step & Time in Stress Loop & Time in Shear Rate Loop \\
\hline Zero instrument & 1 minute & 1 minute \\
\hline Mix and load sample & 1 minute & 1 minute \\
\hline Hold sample & 10 minutes & 2 minutes \\
\hline Ramp shear stress & 5 minutes & 5 minutes \\
\hline Hold at constant shear rate & 1 minute & 1 minute \\
\hline Ramp stress or rate to zero & 5 minutes & 5 minutes \\
\hline Unload sample & 1 minute & 1 minute \\
\hline
\end{tabular}

Note that some hold time is required even in the controlled shear rate flow curve job. This time is present to allow the sample to reach thermal equilibrium. If both a controlled shear rate flow curve and a controlled stress flow curve are generated, then the information in the hold and down ramps tends to be redundant. This is why most of the measurements made in this study skipped the hold and had fairly brief down ramps with the controlled stress up ramp flow curve.

Currently, only the two rheometers at ACTL have the capability to perform this measurement. It is recommended that this capability be included in future rheometers, especially those going into radioactive service. 


\subsection{Constant Shear Rate Time Curves}

The essence of the constant shear rate time curve is a period of time at a constant shear rate. The period of time is of order 10-20 minutes, with 15 minutes serving as a general guide. The test is a point measurement of the thixotropic (or rheopectic) tendencies of a sample. It is a direct test of time dependence under controlled conditions. It is not unlike the hold period between the up and down shear rate ramps of the controlled shear rate flow curve, section 3.1. The principal difference is that the time given to this hold period is much longer than the 30-60 second hold used historically in controlled shear rate flow curve tests.

The selected shear rates were intermediate to the range tested in the controlled shear rate or controlled shear stress flow curve measurements. A shear rate of 200/s was used for most samples tested with the Z41 bob. These were measurements of sludges and SRAT products. A shear rate of 100/s was used for most samples tested with the Z38 bob. These were measurements of SME products and samples with sand and coal. Both shear rate settings were at one-third the maximum shear rate used in most flow curve measurements (0-600/s for Z41 and 0-300/s for Z38). They represented shear rates that should almost always fall in the laminar flow regime for DWPF slurry samples, i.e. would not be disrupted by Taylor vortices as seen in the controlled shear rate flow curves. A few samples were tested at two different shear rates to assess the significance of these choices.

\subsubsection{Time Curve on Viscosity Standard}

The method was tested on a Newtonian viscosity standard expected to have no time dependence. The measurement involved a step jump to 100/s shear rate, a hold at 100/s for 60 seconds, and then a quick ramp down to zero shear rate over 30 seconds. The plot is of the viscosity versus time.

Figure 30. Constant Shear Rate Time Test of NIST N35 Viscosity Standard

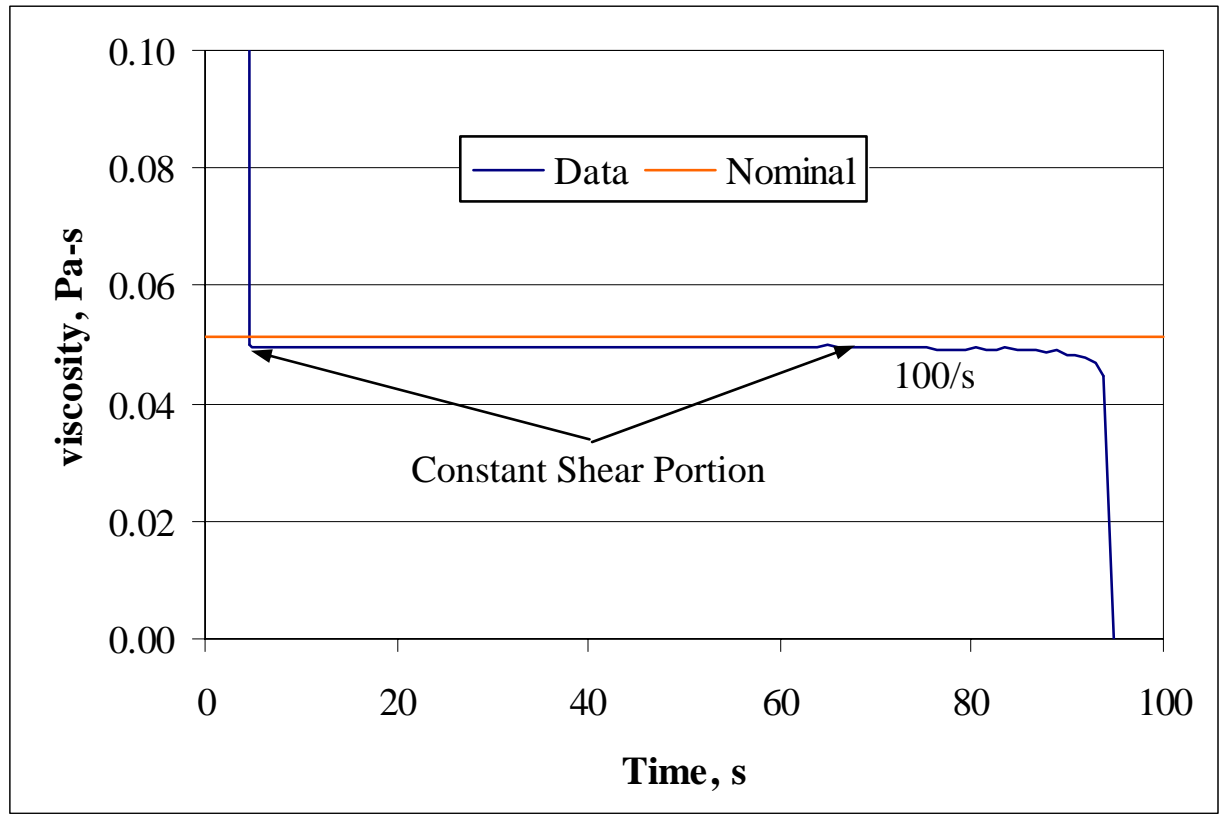

The first point was quite high due to the inertia in the system. Once inertial effects were overcome, which was fairly quickly, the sample tracked quite closely with the nominal viscosity reported by the vendor, Canon. The viscosity fell off during the last few seconds of the down ramp as the shear rate returned to near zero values. 


\subsubsection{Time Curve on Process Samples}

A format was sought to enhance comparisons of the relative time dependence of process samples. This was accomplished by plotting a relative viscosity as a function of time. The relative viscosity was defined as the actual apparent viscosity (ratio of shear stress to shear rate) divided by the average relative viscosity over the duration of the constant shear rate period. A steady result of about one indicates that the apparent viscosity remained essentially constant during the constant shear rate period. A plot showing increasing apparent viscosity from beginning to end would indicate rheopexy, and the opposite trend would indicate thixotropy.

Figure 31 compares the relative viscosity of four sludge simulants. These data were all taken using the Z41 bob. Hold times prior to initiating shear were only long enough to establish thermal equilibrium, since the samples were above the yield stress during the constant shear rate period. All four samples were sheared at a shear rate of 200/s for 15 minutes. There were brief up ramps (5 s) and down ramps (30 s) prior to and following the 15 minute periods at constant shear. That transitional shear rate data was left off of the plot.

Figure 31. Comparison of the Time Dependence of Four Sludge Simulants

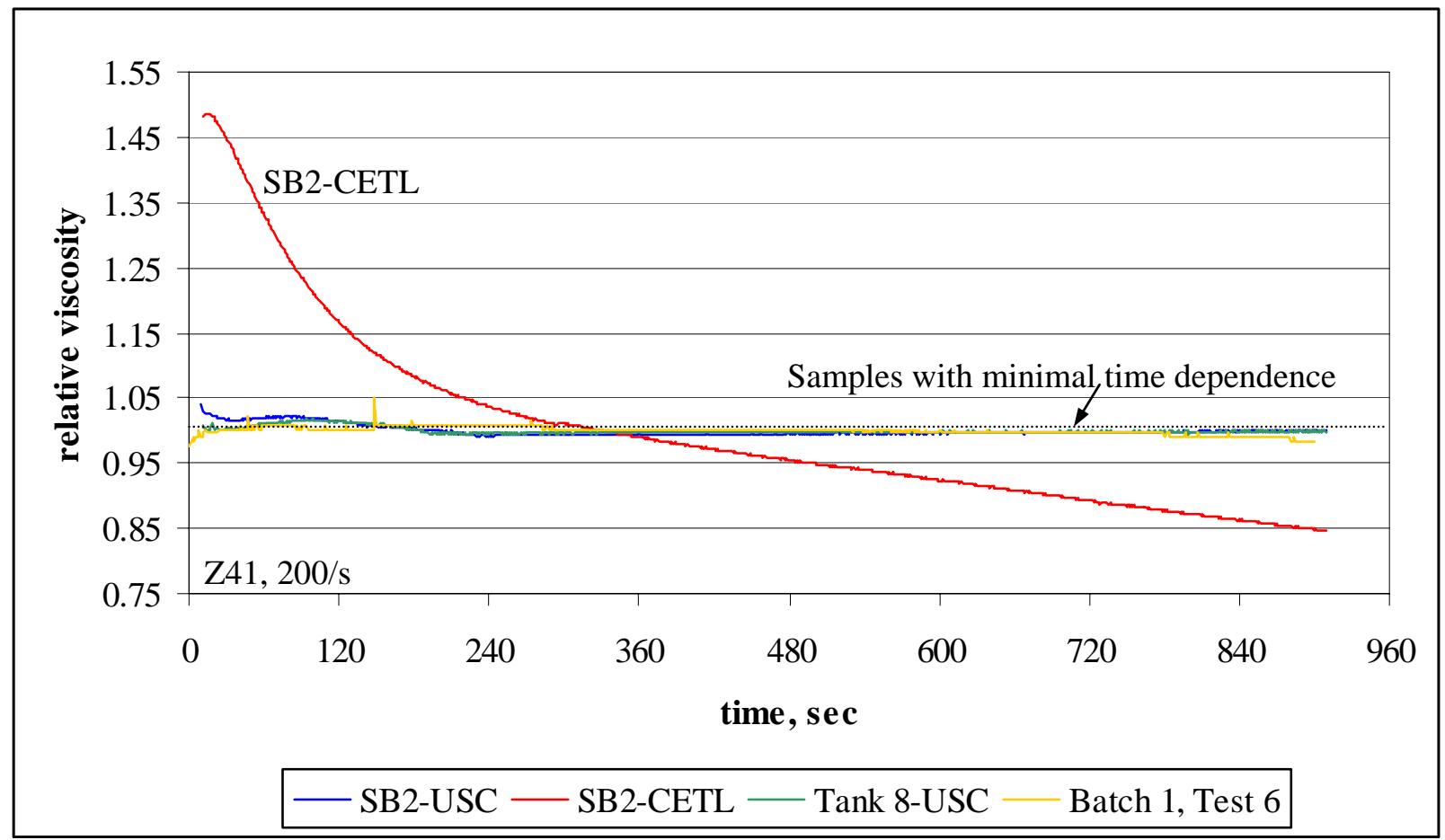

The dominant feature of Figure 31 is the relatively large time dependence of the SB2 CETL sludge simulant. This is the simulant that gave a hump in the controlled shear rate up flow curve. The apparent viscosity fell by about $42 \%$ over fifteen minutes. The other three simulants appear completely time independent in comparison to SB2 from CETL. There are some effects in the first few minutes, however, perhaps due to inertial damping or gel disruption, which were somewhat different for each sample. The average apparent viscosities, $\tau / \dot{\gamma}$, of these four samples at $\dot{\gamma}=200 /$ s are given in Table 6 . 
Table 6. Average Apparent Viscosity at 200/s Shear Rate of Four Sludge Simulants

\begin{tabular}{|l|c|}
\hline Sample & Average Apparent Viscosity \\
\hline SB2 USC Sludge & $25.85 \mathrm{cP}$ \\
\hline SB2 CETL Sludge & $33.25 \mathrm{cP}$ \\
\hline Tank 8 USC Sludge & $19.15 \mathrm{cP}$ \\
\hline Batch 1, Test 6 Sludge & $158.6 \mathrm{cP}$ \\
\hline
\end{tabular}

The Batch 1, Test 6 Optima sludge simulant was quite thick, while the Tank 8 USC sludge was quite thin. The two SB2 simulants were intermediate. The SB2 USC sludge was tested at 100/s shear rate as well. It showed no time dependence at this shear rate either. The SB2 CETL sludge was tested at 300/s shear rate in addition to the above data. A strong time-dependence was indicated at 300/s also. These sludges were fairly insensitive rheologically to the formation of Taylor vortices during a controlled shear rate flow curve, so it seems less likely that the time-dependent behavior seen here was due to solids segregation.

Figure 32 compares data for three samples based on two SRAT products. The SRAT product made from CETL SB2 simulant was analyzed as made and as decanted to higher total solids per Table 1 . The SB2 data were obtained using the Z41 bob at a shear rate of 200/s. The SB2/3 SRAT product data were obtained using the Z38 bob at a shear rate of 100/s. Z38 was used because of the presence of sand and coal in the SB2/3 sample.

Figure 32. Comparison of the Time Dependence of Three SRAT Products

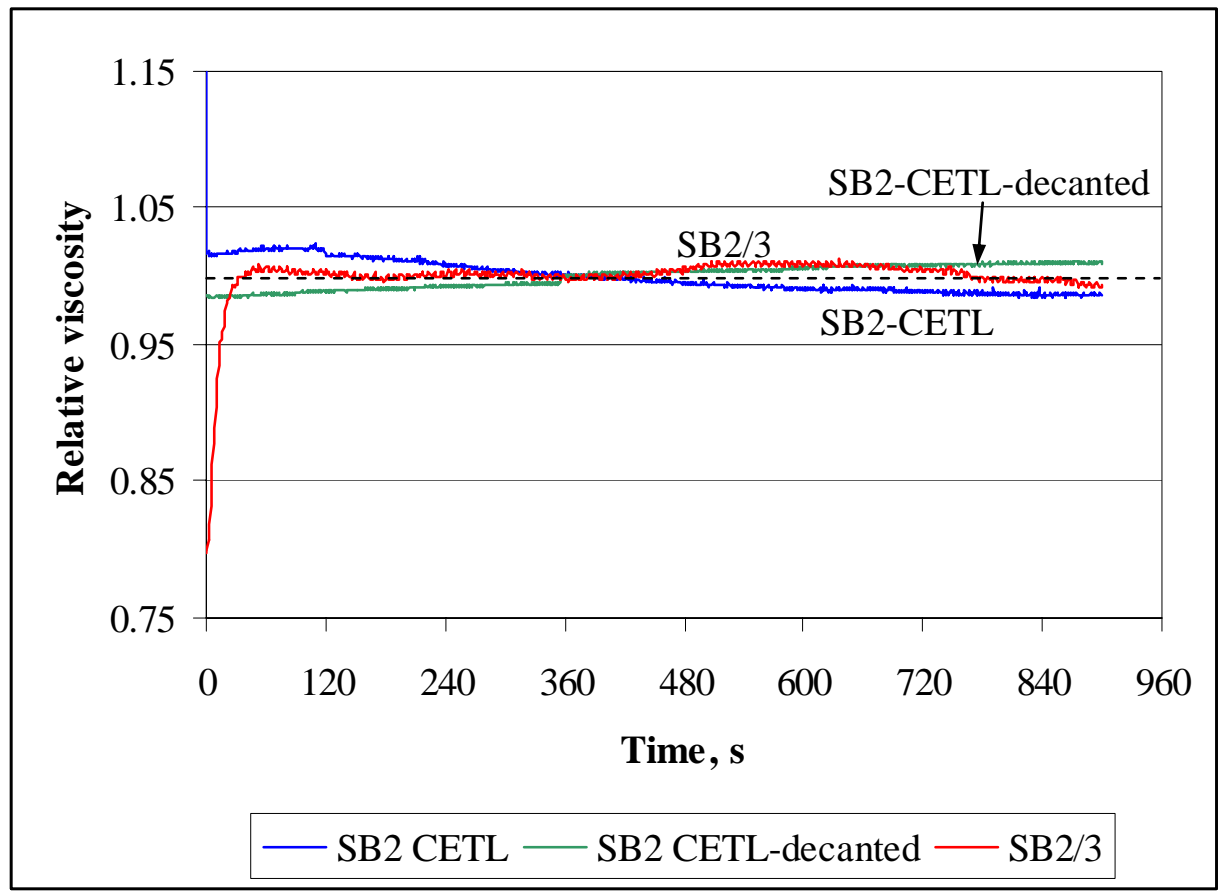

The average apparent viscosities from the three tests are given in Table 7. 
WSRC-TR-2004-00334

Revision 0

Table 7. Average Apparent Viscosity of Three SRAT Products

\begin{tabular}{|l|c|c|c|c|}
\hline SRAT Product: & $\begin{array}{c}\text { Avg. Apparent } \\
\text { Viscosity }\end{array}$ & $\begin{array}{c}\text { Nominal } \\
\text { Shear Rate }\end{array}$ & $\begin{array}{c}\text { Wt. \% Total } \\
\text { Solids }\end{array}$ & $\begin{array}{c}\text { Wt. \% } \\
\text { Insoluble } \\
\text { Solids }\end{array}$ \\
\hline SB2-CETL & $6.54 \mathrm{cP}$ & $200 / \mathrm{s}$ & 12.6 & 8.03 \\
\hline SB2-CETL decanted & $29.2 \mathrm{cP}$ & $200 / \mathrm{s}$ & 17.5 & 12.3 \\
\hline SB2/3 CETL & $28.6 \mathrm{cP}$ & $100 / \mathrm{s}$ & 25.4 & 12.8 \\
\hline
\end{tabular}

The three SRAT product samples showed essentially negligible time dependence. Initial effects seemed to take longer to die out with the Z38 measurement (SB2/3) than with the two Z41 measurements. The SB2 CETL SRAT product appeared to be mildly thixotropic, but it was quite thin. There may have been some slight settling of the slurry particles during the fifteen minute test. The same sample, after decanting it to raise the solids content and yield stress, appeared to be mildly rheopectic. Such data raise the question of whether or not the time dependence of the slurry sample rheological properties depends on the concentration of solids. The SB2/3 SRAT product data looks a little unsteady. This may be due to partial settling of sand and coal, or perhaps just rearrangements of these particles into lower drag orientations.

Figure 33 compares the relative viscosity of five simulant SME products. These data were all taken using the Z38 bob. Hold times were short, since the sample would be fully yielded during the constant shear rate period. All five samples were sheared at a shear rate of 100/s for at least 15 minutes. There were brief up ramps (5 s) and down ramps (30 s) prior to and following the constant shear rate period. The transitional shear rate data was left off of the plot. A data point was taken each second of the measurement. 
Figure 33. Comparison of the Time Dependence of Five SME Products

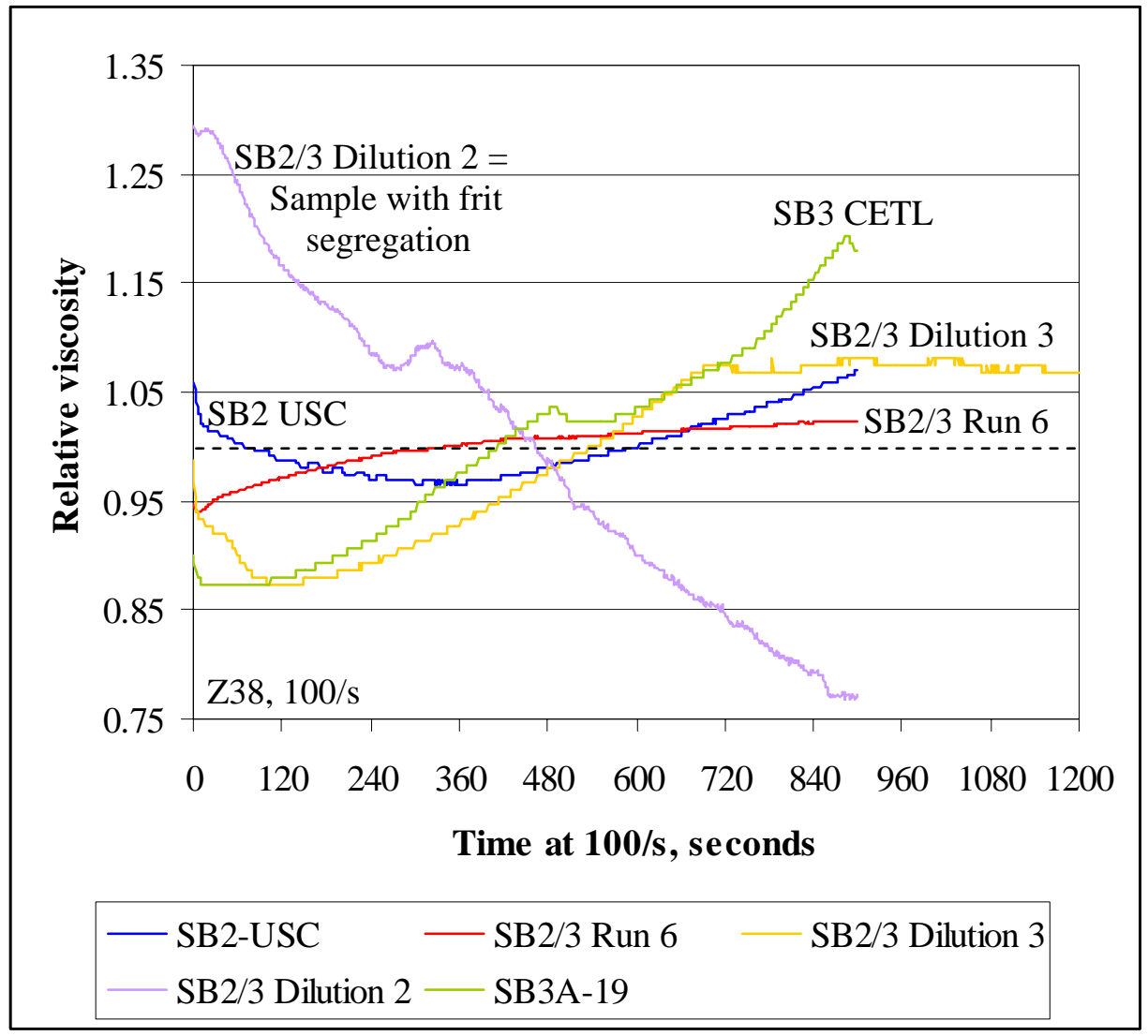

This is clearly a very different graph from the one for the sludge simulants, Figure 31, or the one for the SRAT products, Figure 32. Many different types of behavior were seen with SME products. This was not entirely unexpected, since most of the recent unusual rheological data had been coming from SME products. The main exceptions were the hump in the SB2 CETL sludge simulant and occasional cases of flow curve cross-over.

The average apparent viscosities at 100/s shear rate for these five SME products are given in Table 8 .

Table 8. Average Apparent Viscosities of SME Products

\begin{tabular}{|l|c|c|c|c|}
\hline SME Sample: & Viscosity & $\begin{array}{c}\text { Wt. \% Total } \\
\text { Solids }\end{array}$ & $\begin{array}{c}\text { Wt. \% } \\
\text { Insoluble } \\
\text { Solids }\end{array}$ & $\mathrm{pH}$ \\
\hline SB2 USC & $267 \mathrm{cP}$ & 49.3 & 43.3 & 7.98 \\
\hline SB2/3 Run 6 & $460 \mathrm{cP}$ & 54.4 & n.a. & n.a. \\
\hline SB2/3 Dilution 3 & $148 \mathrm{cP}$ & 41.5 & n.a. & n.a. \\
\hline SB2/3 Dilution 2 & $31.9 \mathrm{cP}$ & 29.5 & n.a. & n.a. \\
\hline SB3 CETL & $147 \mathrm{cP}$ & 54 & 37 & 7.67 \\
\hline
\end{tabular}

These five samples were much more viscous than the four sludge simulants or three SRAT products. Consequently, any inertial effects were expected to be damped out much faster here than in the sludge data. 
The SB2/3 Dilution 2 SME product sample from the heel study showed considerable thinning with time (apparent thixotropy). Testing outside the rheometer indicated that this sample did not suspend frit very well on a time scale of ten minutes in a graduated cylinder. The next two least viscous samples were SB3 CETL from the SB3 simulant flow sheet study in support of the Shielded Cells qualification and Dilution 3 of the SB2/3 SME product from the heel study. These two samples were visually thicker than the Dilution 2 sample. Segregation of SB3 CETL was studied by separately collecting sample that poured from the cup and comparing it to fresh material. The result was that no evidence of lower solids was found. SB3 CETL was tested at 200/s as well as 100/s shear rate. The resulting curve was very similar, except that it was shifted to a higher shear stress. SB2/3 Dilution 3 and SB3 CETL SME products both appeared to be mildly rheopectic, i.e. thickening with time under shear.

The test on the Dilution 3 sample was allowed to continue for an extra five minutes with a surprising result. The apparent end to the rising viscosity at 12 minutes continued past 15 minutes until the end of the test at 20 minutes. This indicates that this sample had stabilized at this shear rate to an essentially constant apparent viscosity. Unfortunately, it took 12 minutes for this to happen. That is close to the length of a controlled shear rate flow curve measurement. SB3 CETL SME product apparently peaked at about 14 minutes, 40 seconds, and then began to fall during the last 20 seconds (last 20 data points).

The SB2 SME product made from USC simulant was unusual. It thinned initially and then thickened later. This sample gave cross-over in the controlled shear rate flow curve measurement, section 3.1.3. This reversal of rheological tendency seen in the constant shear rate time curve may well tie into the explanation for the cross-over phenomenon and be the real explanation for why the controlled rate flow curve looked that way. Note that variations in apparent viscosity for this sample stayed within $5 \%$ of the mean for nearly the entire 15 minutes. This sample did not have much time dependence in an overall sense. The time dependence that it did have though was unusual. Such data suggest that there may be competing phenomena with different time constants present, e.g. one that tends to decrease viscosity and one that tends to increase viscosity. The one that tends to decrease viscosity was faster, but it was overcome in the long run by the competing effect that tended to increase viscosity.

The SB2/3 CETL SME product from Run 6 for the SMRF at 155\% acid stoichiometry was the thickest in the set and well above the DWPF design basis window. It exhibited a mildly rheopectic nature that appeared to be leveling off with time. The apparent viscosity changed only 3\% during the last ten minutes of the constant shear rate period. This was the most stable of the five SME products in a relative sense.

\subsubsection{Assessment}

The constant shear rate time curve was a very easy measurement to perform. The only operational drawback was that it was somewhat more time consuming than the typical 11 minute controlled shear rate flow curve measurement. The primary analytical drawback was that it cannot substitute for a flow curve measurement in generating the Bingham plastic model yield stress and consistency values needed in certain calculations. Choice of shear rate did not appear to be critical to the results obtained, as long as the slurry was kept moving fast enough to help suspend solids, while avoiding the region of Taylor vortices. This test can be performed with all of the rheometers currently in use by ITS, including those in radioactive service.

The three SRAT product simulants and three of the four sludge simulants showed negligible time dependence. Previous rheological measurements of real waste sludges have also indicated negligible time dependence. Rheological measurements of radioactive SB2 SRAT products showed at most a hint of thixotropy (down ramps just slightly below up ramps in the five flow curve measurements). 
All of the non-settling simulant SME products tested, however, showed some indication of rheopectic behavior. The extent was not always particularly large, but it was there. This may not be true rheological behavior, but may instead indicate that the insoluble solid fraction is segregating within the annular gap of the instrument as time passes. Unfortunately, there is almost no comparable radioactive data on SME products. What data that exist were taken with the MV1 bob that corresponds more closely to Z41. It is strongly recommended that more radioactive SME product rheology data be obtained in the near future. SRAT data using both Z41 and Z38 indicate that there is a sensitivity of the data to the bob selection. The Z41 bobs are not used on simulant SME products because the much greater sample load would soon etch the surface of the bobs with scratches. The vendor recommends replacement when the surface becomes significantly scratched. 


\subsection{Other Rotational Measurements}

Several other methods were investigated that involve the direct rotation of the cylindrical bob in a single direction. These included the small deformation yield point determination by two methods, controlled shear and controlled deformation, as well as creep-recovery measurements. These studies happened concurrently with the early investigations into the controlled stress ramp and the oscillation tests.

One potential method was not investigated, because of the information being obtained from the constant shear rate time curve tests. This is a variation of the controlled shear rate flow curve measurement. In this variant, the shear rate is not ramped continuously. Instead, the shear rate is ramped in incremental steps, e.g. 30/s, 60/s, 90/s, ..., 300/s. The sample is held at each shear rate until the rate of change of the shear stress with respect to time falls below some test cut-off criterion. This method eliminates inertial effects associated with changing the momentum of the bob and sample during the continuous ramps by allowing the sample to come to a steady momentum state at each shear rate before recording the shear stress. It is slightly more precise than the method currently being used. It generates far less data, however, so the data needs to be smooth to get a good fit to the Bingham plastic equation. Also, it is necessary to define a criterion for stepping to the next shear rate. If this is routinely chosen such that most time-dependent behavior seen to date would be eliminated, then the time to perform this test could become prohibitively long. If this choice is not made, then the same issues would appear in these flow curves that appear in the controlled shear rate flow curves currently being made.

\subsubsection{Small Deformation Yield Point Determination}

The method is designed to find the true yield stress of a sample. The bob is slowly rotated and the shear stress is recorded. Ideally, the bob is rotated at a slow constant speed. Practically, the rheometer controls the rotation of the bob by varying the current to the motor. This is most directly a control of the torque, which is related to shear stress, rather than of the speed, which is related to shear rate. For the rheometer to control speed requires the use of feedback control logic. This requires measurement of the bob position to high accuracy as a function of time. This is difficult when the bob is barely moving.

Two modes were tested. One mode failed to produce good data. This was a mode that attempted to ramp the deformation (strain) linearly with time. The ramp in strain was not generated properly by the rheometer (RS150 and RS600). The second mode attempted to ramp the deformation linearly with time by setting a constant shear rate (the time derivative of deformation). The reported shear rates were not constant with time as commanded, however the sample stress did appear to ramp linearly with time as desired. Various shear rates were specified, often with no effect on what the rheometer actually did. This in and of itself is not critical if the stress ramps linearly with time.

During this test, the shear stress is gradually increasing. At some point the sample yields in the sense of transitioning from solid-like to fluid-like. Past this point, the shear stress no longer rises linearly with time. Typical data are shown in Figure 34. 
Figure 34. Direct Yield Point Measurement Data for SB3 CETL SME Product

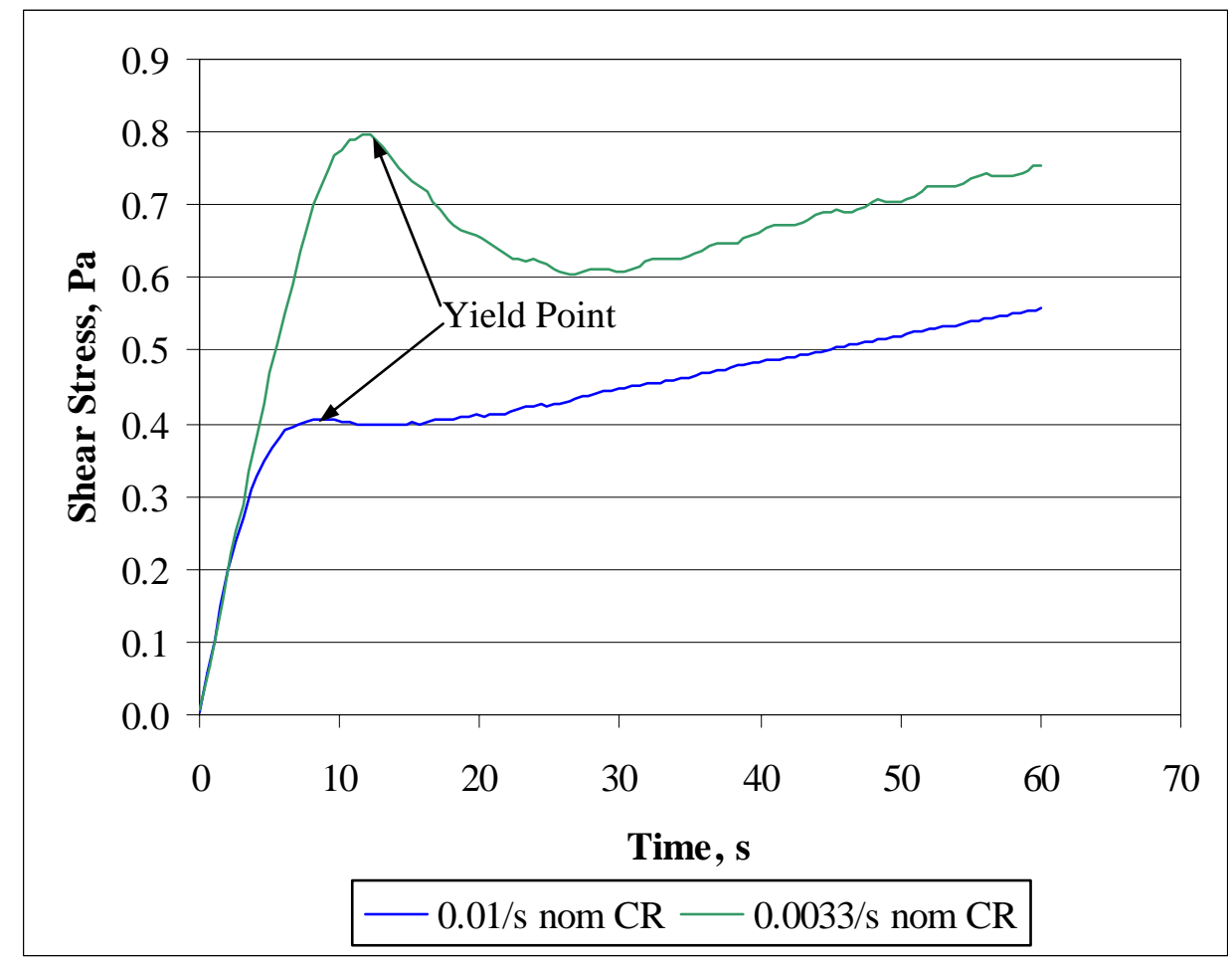

The shear rate was controlled at two different speeds shown in the legend. The results obtained appeared to depend on the speed selected. Data obtained with SB2/3CETL SME product from Run 6 at 155\% acid gave similar variations with shear rate setting, as well as variations indicating no reproducibility when using the same shear rate. Further investigation revealed that the time the sample was held in the annular gap before initiating the measurement had a significant impact on the result. It was also noted that the shear stress at the yield point was generally higher than that being obtained by other tests (about $0.3 \mathrm{~Pa}$ for SB3A-19). The test did confirm that the true yield stress of the samples tested was approximately the value associated with the first transition in the strain-stress data generated by the controlled shear stress ramp. This test was abandoned, because it appeared to provide less information than other tests, coupled with being difficult to perform reproducibly. The controlled shear stress test with an adequate initial hold appeared to offer a superior alternative to this test.

\subsubsection{Creep-Recovery Measurements}

The creep-recovery test is another approach to determining the true yield stress of a sample. It is more time consuming than the other tests described, but is considered to be one of the most accurate methods for evaluating the yield phenomenon. The test is performed by loading the sample as in all of the other tests. After some period of time, a single shear stress is applied to the sample for a fixed period of time, e.g. 30 seconds. This applied stress produces a small deformation (called creep). At the end of the creep period, the stress is removed. If the sample has elasticity, then the deformation will be partially or totally reversed as the sample attempts to recover its original position. A high percentage recovery of the bob position from its deformed position back to its original position indicates that the sample did not yield at the stress applied. A low percentage recovery indicates that the sample flowed and no longer remembers its original position. The test must be repeated at various shear stress settings to generate enough data to determine the location of the true yield stress. It is therefore a time consuming test. Some additional 
material parameters are generated, e.g. a recovery time constant and a zero shear rate viscosity, which may or may not have some value.

An example of the data obtained is given in Figure 35.

Figure 35. SB2/3 SME Product, Run 6, 155\% Acid - Creep-Recovery Data, Z38

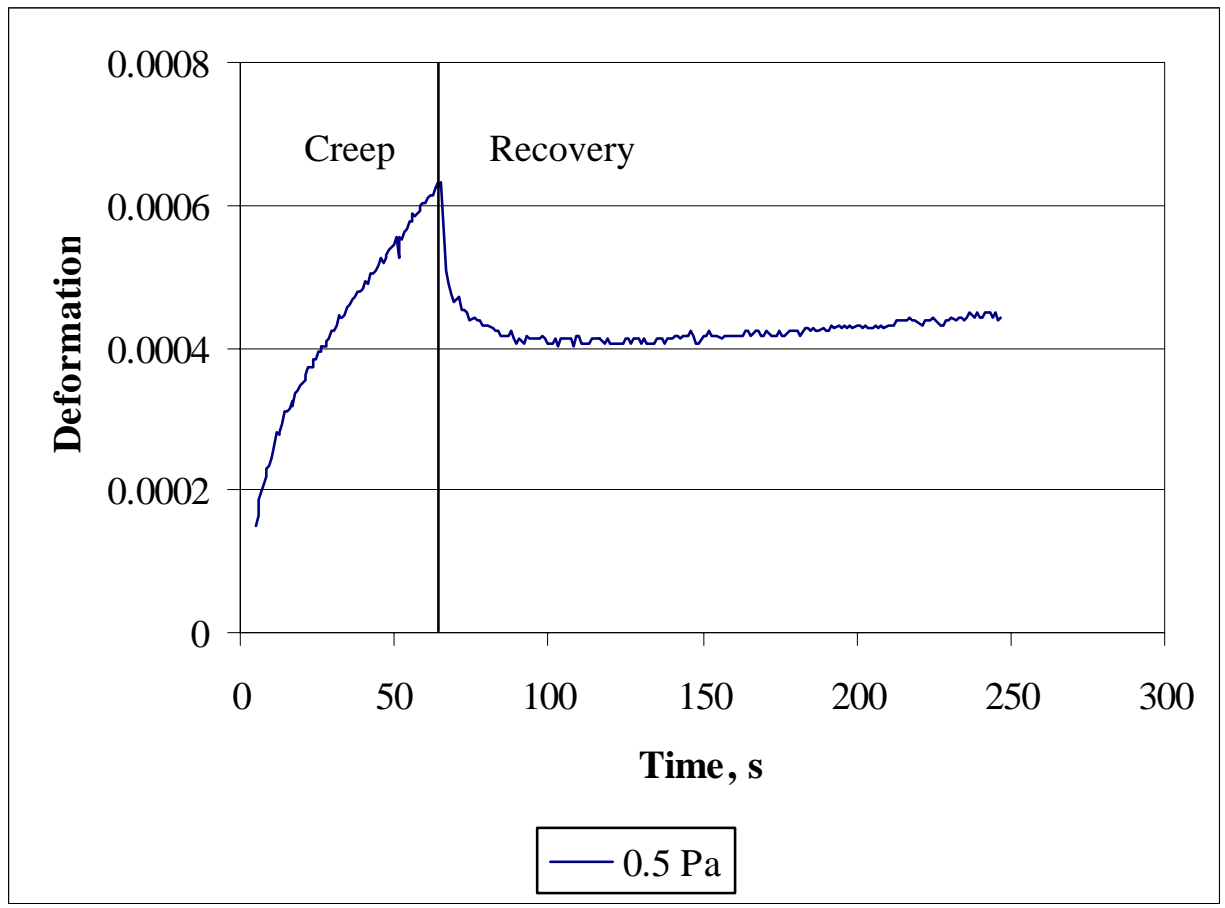

This test would indicate that this sample did not recover sufficiently to be considered below the true yield stress. This was seen in all early applications of this test at almost any stress selected. When data was compared to the other methods, this test indicated that the sample had yielded when the other tests indicated that the sample had not yielded.

It was found that this test is also very sensitive to the initial hold time, during which the gel structure reforms. This was seen in the other tests that can examine the true yield stresses of the slurries. This test, however, is particularly sensitive. The data in Figure 36 show how sensitive this test is. 
Figure 36. Creep-Recovery Data for SB2 USC Sludge Simulant, Z41

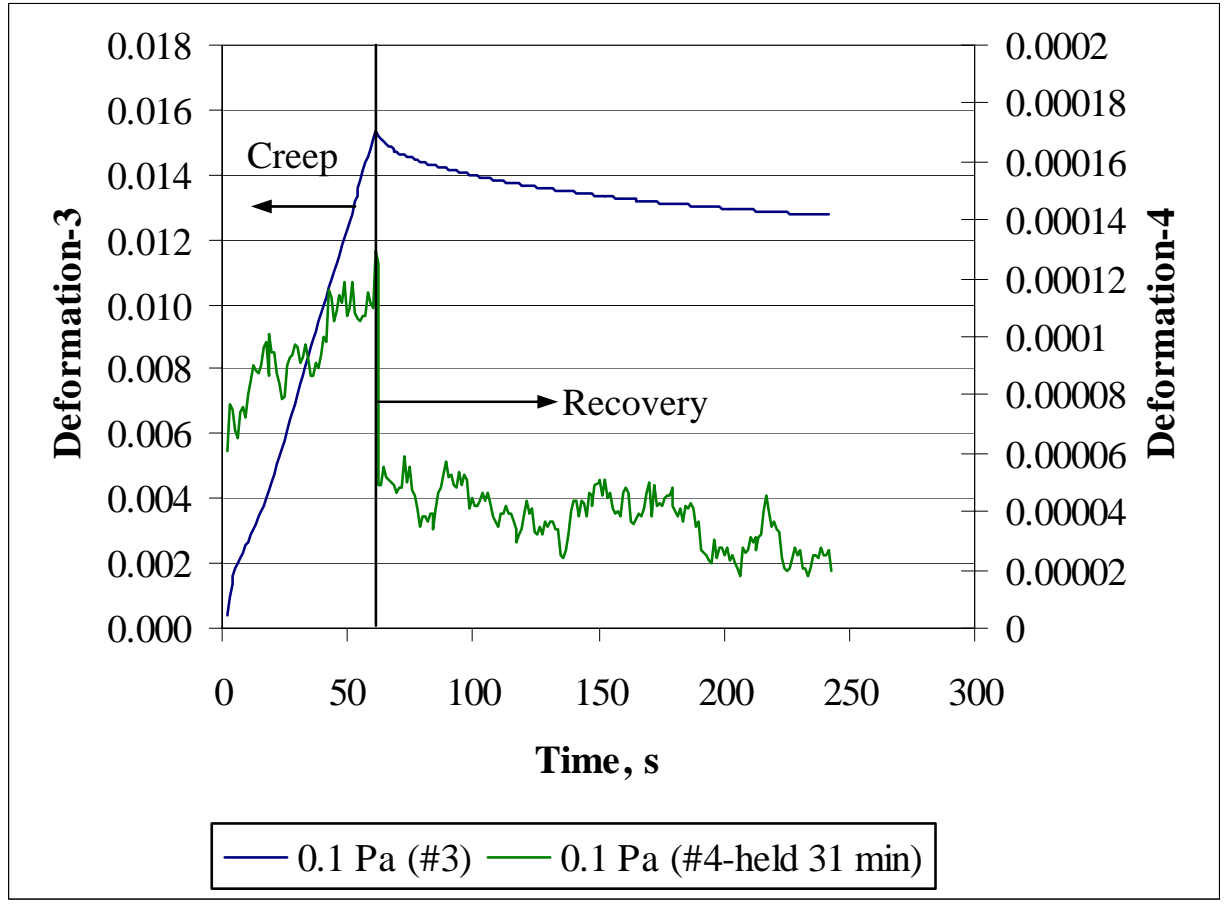

A stress of $0.1 \mathrm{~Pa}$ was well below the indicated true yield stress by other tests on SB2 USC sludge simulant. The data indicate a fairly high recovery of the original position in the sample that was held for 31 minutes. This material was fairly slow settling, or this test would not have been attempted. Another indication that this sample is below the true yield stress is the almost instantaneous recovery of $2 / 3$ of the original position once stress was removed. The recovery of the sample not held for any appreciable time prior to applying the stress was much less (in a percentage sense), and the deformation obtained in 60 seconds was two orders of magnitude greater. This is further confirmation that the gel-structures in the simulant samples are fragile and slow to reform. They are described as fragile, because loading the rheometer appears to destroy them quite thoroughly.

This test was dropped from further consideration as it became apparent that prohibitively long hold times would be required to obtain the individual measurements on a sample. The test was pushed to the limit of practical application and slurry stability. Assuming that five to ten tests would be required to determine the true yield stress location for a given sample, coupled with a need for hold times approaching a half an hour, led to the conclusion that a half day could be spent determining the true yield stress of a single sample. This did not appear to be a good use of available resources. (ThermoHaake's technical representative had referred to this test as more of a university test, meaning that university researchers are the only ones with the patience and the need for this level of characterization.) 


\subsection{Oscillatory Measurements}

Oscillatory measurements are fundamentally different from the direct rotational methods discussed in sections 3.1 to 3.4. Oscillatory measurements are what the name implies, i.e. various tests where the bob is oscillated clockwise and counter-clockwise about a fixed position. As such, these tests do not produce well-defined streamlines of fluid motion, while the rotational tests do produce smooth circular streamlines during laminar flow. The presence of streamlines affects the orientation of solid particles. Particles prefer to orient into a minimum drag configuration with respect to the streamlines around them. Streamlines beneath a particle also tend to support a particle and inhibit settling. Consequently, it was initially unclear how results obtained by oscillatory measurements would compare to results obtained by rotational methods.

Three primary measurement methods were developed in this study. These were:

- the oscillation stress sweep, also known as the oscillation amplitude sweep,

- the oscillation frequency sweep

- the oscillation time sweep

The independent parameters in these methods are the oscillation frequency, the amplitude of the applied stress, and the duration/ramp rate. Each test varies one parameter while holding the other two constant. The stress sweep varies the shear stress at constant frequency over a fixed ramping interval of shear stress. The oscillation frequency sweep varies the oscillation frequency at a constant shear stress over a fixed ramping interval of frequency. The oscillation time sweep holds the oscillation frequency and shear stress constant and monitors the response for a specified time interval.

The response of all three tests is a deformation oscillation. The oscillation is typically out of phase with the input stress oscillation. The magnitude of the deformation oscillation varies with the magnitude of the stress oscillation. These oscillation magnitudes and the phase shift are used to evaluate the rheological properties as described in section 2.2.4.6.

\subsubsection{Oscillation Stress Sweep Measurements}

This test gives data that are the most comparable to the rotational methods. Consequently, it was a leading candidate for development. The shear stress range studied was generally set to span the region from below the true yield stress to above the Bingham plastic yield stress (when these were known). Results were found to be sensitive to the initial sample hold time. This sensitivity was found in other methods, e.g. section 3.2.2.

Most testing was done at a frequency of $1 \mathrm{~Hz}$. All that is required, however, is a suitable frequency. Frequencies up to $4 \mathrm{~Hz}$ were tested. As frequency increased, a sample will tend to react more like a rigid body. This is not desired when characterizing fluid properties. The main reason for increasing frequency is to speed up the test. Frequencies down to $0.25 \mathrm{~Hz}$ were tested. Testing at $0.25 \mathrm{~Hz}$ was considerably slower than testing at $1 \mathrm{~Hz}$. The data being obtained at $0.25 \mathrm{~Hz}$ did appear to be a little smoother. Conversely, some of the data obtained at $4 \mathrm{~Hz}$ was too noisy.

Normal practice is to perform at least one oscillation frequency sweep measurement, section 3.5.2, at a shear stress below the yield stress that covers the frequency planned for the oscillation stress sweep. This check is made to confirm that the selected frequency is not close to an ambiguous region. Samples can be forced to switch from solid-like to liquid-like behavior by varying either frequency or stress. If the 
sample is about to transition at the selected frequency for the oscillation stress sweep, then it is advisable to switch to a lower frequency. As discussed in section 3.5.2, however, no transitions were observed near $1 \mathrm{~Hz}$ on any of the samples.

\subsubsection{Oscillation Stress Sweep on Viscosity Standard}

The oscillation stress sweep on the N35 viscosity standard was done primarily to test the method. Figure 37 shows one of the possible graphs that can be produced by this method. This is actually two graphs. Normally the storage and loss moduli, G' and G”, are plotted together. Delta does not need to be on the same graph, and contains much of the same information about the sample behavior except for the magnitude effects.

Figure 37. Oscillation Stress Sweep on NIST N35 Viscosity Standard

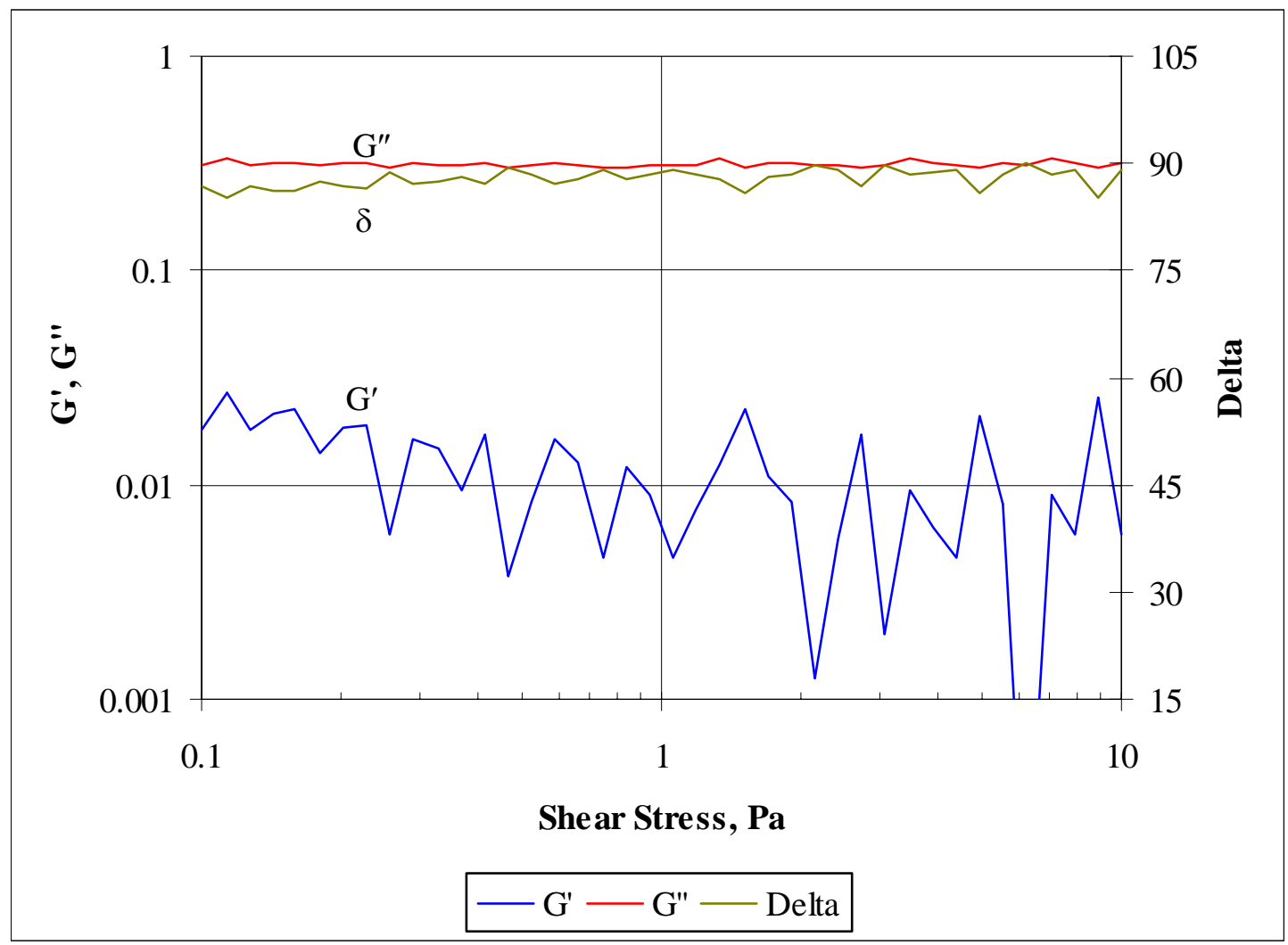

G” >> G' indicates a strong liquid-like nature to the sample over the range of stresses tested. G' is mostly computational noise from the conversion of the deformation lag information into G' and G”. Delta, $\delta$, is $\operatorname{Arctan}\left(G^{\prime \prime} / G^{\prime}\right)$. Delta is in degrees in the plots presented, not in radians, e.g. $90=\pi / 2$ radians. Since both G' and G" are not negative, $\delta$ is limited to the interval from 0 to 90 degrees. 90 degrees is equivalent to $100 \%$ liquid-like, while 0 degrees is equivalent to $100 \%$ solid-like. The viscosity standard ran with an average $\delta$ of about 88 degrees, which was essentially independent of shear stress.

\subsubsection{Oscillation Stress Sweeps on Process Samples}

Data from an oscillation stress sweep can be used to produce several different graphical products, much like the controlled shear stress flow curve. An example of one product is given in Figure 38 for one of the SME products from the SB2/3 acid stoichiometry study (Baich, 2004a). The measurement was made 
using the Z38 bob. The sample was held for 10 minutes in the instrument before initiating the oscillating stress ramp.

Figure 38. Oscillation Stress Sweep on SB2/3 CETL SME Product (170\% Stoichiometry), Z38

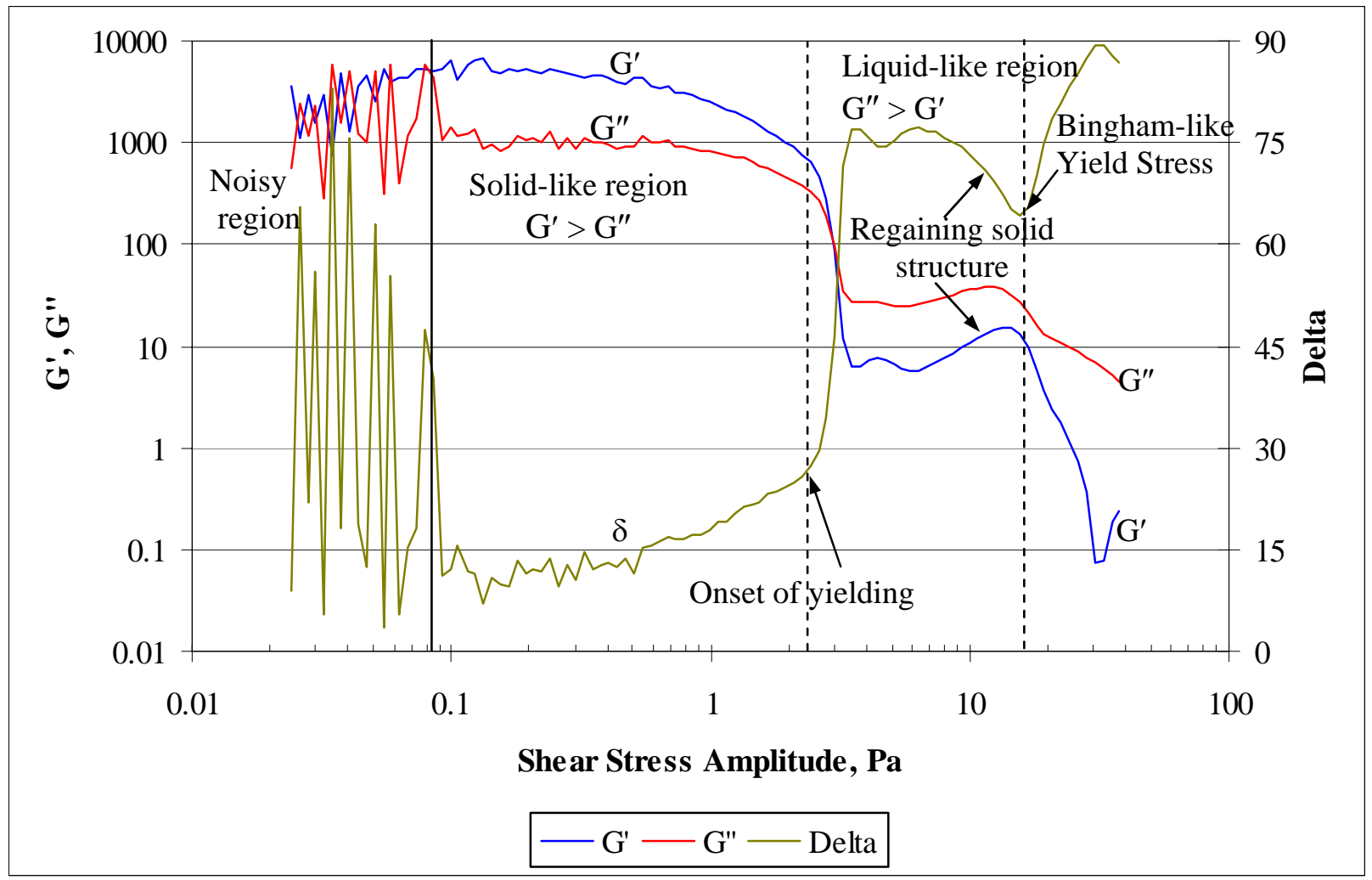

G' is the elastic modulus. G” is the loss modulus. The RheoWin rheometer control software gives a realtime graph of the deformation response superimposed on the sinusoidal input stress oscillation. The deformation response does not look like a sinusoidal wave at the low stresses in the noisy region marked on Figure 38. There are some adjustable parameters in the software that might remove some of the noise, but this would be at the expense of increased measurement time. Since the data are smooth prior to the true yield stress, there does not seem to be any need to do this.

The noisy data in Figure 38 between $0.02 \mathrm{~Pa}$ and $0.08 \mathrm{~Pa}$ were common in the low shear stress range with thick SME products. These data could easily be excluded from the graph, but are shown here as an example of what happens with the method in the low stress limit. From $0.02 \mathrm{~Pa}$ to about $3 \mathrm{~Pa}$, the elastic modulus, G', exceeded the loss modulus, G”. The standard interpretation is that the sample is more solidlike than liquid-like when this is the case. $\delta$ is less than 45 degrees for this same range. Yielding seemed to coincide not with $\delta$ hitting 45 degrees, but with $\delta$ starting to shoot up (marked "Onset of yielding” in Figure 38. There was a region after the sample has yielded where it temporarily regains some of its solid structure ( $G$ ' increases and $\delta$ decreases), though it remained predominantly liquid-like. This was followed by another yield-like transition that corresponds closely with the Bingham plastic yield stress obtained by rotational methods.

Power law fits to the controlled shear stress ramp data, Figure 27, for this same sample gave a true yield stress of 2.7 $\mathrm{Pa}$ and an upper yield stress of $25 \mathrm{~Pa}$. A traditional Bingham plastic flow curve fit gave a 
yield stress of $24 \mathrm{~Pa}$. The $\delta$ transitions in Figure 38 are at 2-3 Pa and at 15-18 Pa. These are comparable to the rotational data at the true yield stress, but lower for the Bingham yield stress.

The oscillation stress sweep data can also be plotted as the log of the deformation amplitude versus the log of the shear stress wave amplitude.

Figure 39. Oscillation Stress Sweep Data for SB2/3 CETL SME Product (170\% Acid), Z38

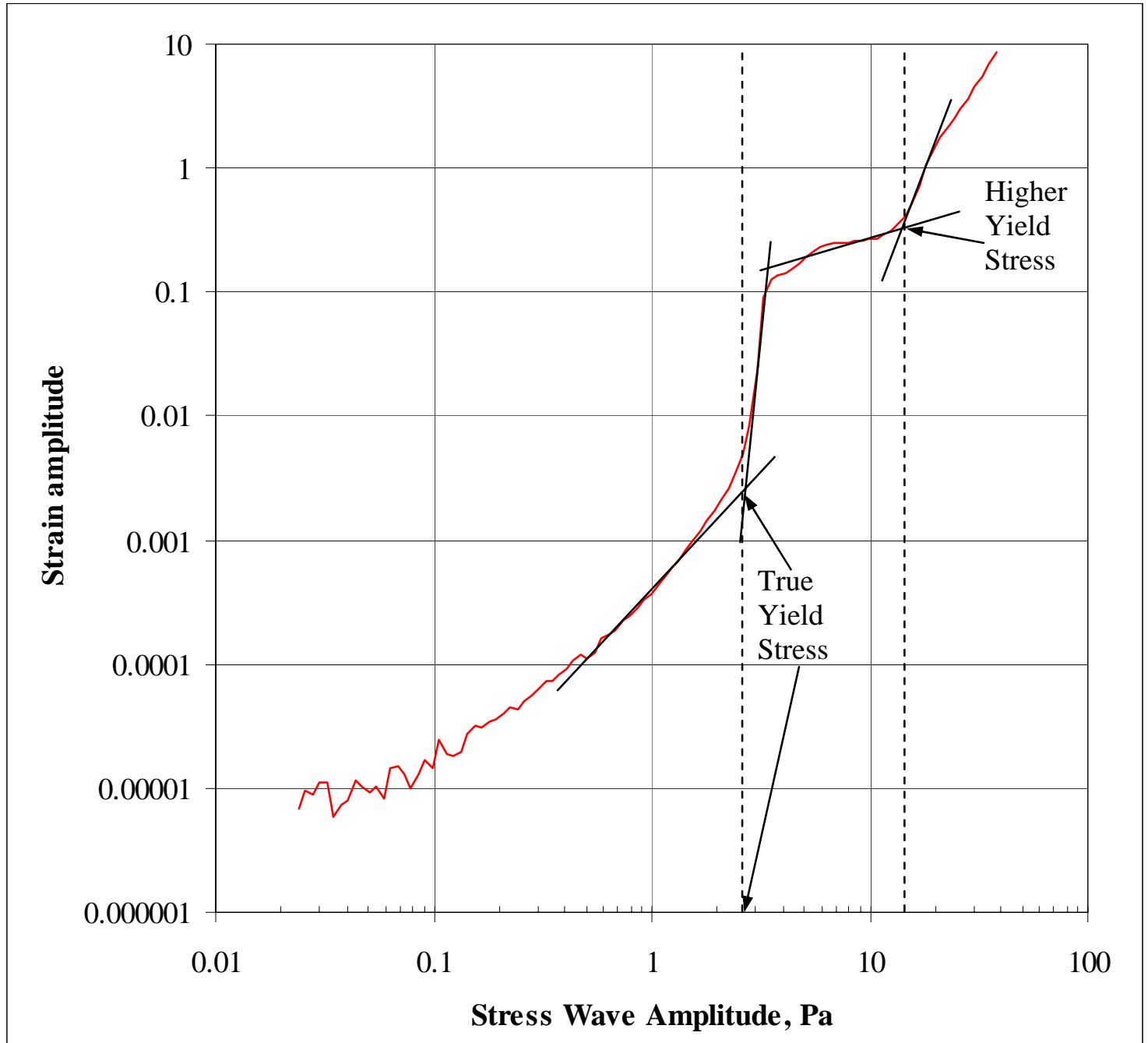

Graphical analysis of the strain-stress wave data gives the true yield stress at about 2.8 Pa and the higher yield stress at about 13-15 Pa. This is virtually the same true yield stress as that obtained by the controlled shear stress ramp. The upper yield stress agrees between Figure 38 and Figure 39, but is lower than the Bingham plastic yield stress. This is most likely because the Bingham plastic yield stress is not exactly what is being measured in this region.

Table 9 below compares SME product yield stress data obtained from the controlled shear stress ramps with similar data from the oscillation stress sweeps. Most of the data has been limited to cases where the sample was held for at least ten minutes in the instrument before initiating stress. The oscillation data was also limited to data at $1 \mathrm{~Hz}$. 
WSRC-TR-2004-00334

Revision 0

Table 9. SME Yield Stress Comparison of Controlled and Oscillation Stress Methods

\begin{tabular}{|l|c|c|c|c|}
\hline Sample: & $\begin{array}{c}\text { True Yield Stress } \\
\text { Controlled Stress }\end{array}$ & $\begin{array}{c}\text { True Yield Stress } \\
\text { Oscillation }\end{array}$ & $\begin{array}{c}\text { Bingham Yield } \\
\text { Stress } \\
\text { Controlled Stress }\end{array}$ & $\begin{array}{c}\text { Upper Yield Stress } \\
\text { Oscillation }\end{array}$ \\
\hline SB2 USC & $4.1 \mathrm{~Pa}$ & $2.3 \mathrm{~Pa}$ & $27 \mathrm{~Pa}$ & $20 \mathrm{~Pa}$ \\
\hline SB2/3 $140 \%$ & $3.8 \mathrm{~Pa}$ & $3 \mathrm{~Pa}$ & $27 \mathrm{~Pa}$ & $20 \mathrm{~Pa}$ \\
\hline SB2/3 $155 \%$ & $0.95,1.0 \mathrm{~Pa}$ & $0.9 \mathrm{~Pa}$ & $11,15 \mathrm{~Pa}$ & $8 \mathrm{~Pa}$ \\
\hline SB2/3 170\% & $2.7 \mathrm{~Pa}$ & $2.8 \mathrm{~Pa}$ & $25 \mathrm{~Pa}$ & $15 \mathrm{~Pa}$ \\
\hline SB2/3 185\% & $0.8,1.5 \mathrm{~Pa}$ & $0.7 \mathrm{~Pa}$ & $13,15 \mathrm{~Pa}$ & $4.7 \mathrm{~Pa}$ \\
\hline Run 5, Dil. 2 & Indeterminate & $0.35 \mathrm{~Pa}$ & $3 \mathrm{~Pa}$ & $1.1 \mathrm{~Pa}$ \\
\hline SB2/3 Run 6 & $2 \mathrm{~Pa}$ & $1 \mathrm{~Pa}$ & $38 \mathrm{~Pa}$ & $25 \mathrm{~Pa}$ \\
\hline SB3 CETL & $0.3,0.4 \mathrm{~Pa}$ & $0.4 \mathrm{~Pa}$ & $6-8 \mathrm{~Pa}$ & $9 \mathrm{~Pa}$ \\
\hline
\end{tabular}

There was pretty good agreement between the true yield stress values obtained by the two methods. The true yield stress values in the table are not very precise, because of the sensitivity to the technique used. Only one oscillation stress sweep upper yield stress was larger than the corresponding Bingham plastic yield stress. This biased trend may be an artifact of the fitting process used to obtain the Bingham plastic yield stress. Other yield stress rheological equations typically give a lower yield stress than the Bingham plastic equation when applied to flow curves for shear-thinning samples like the DWPF slurry samples.

Figure 40 shows the parameter, $\delta$, for the four SB2/3 CETL SME products from the acid stoichiometry study. 
Figure 40. SB2/3 CETL SME Products - Delta Comparison

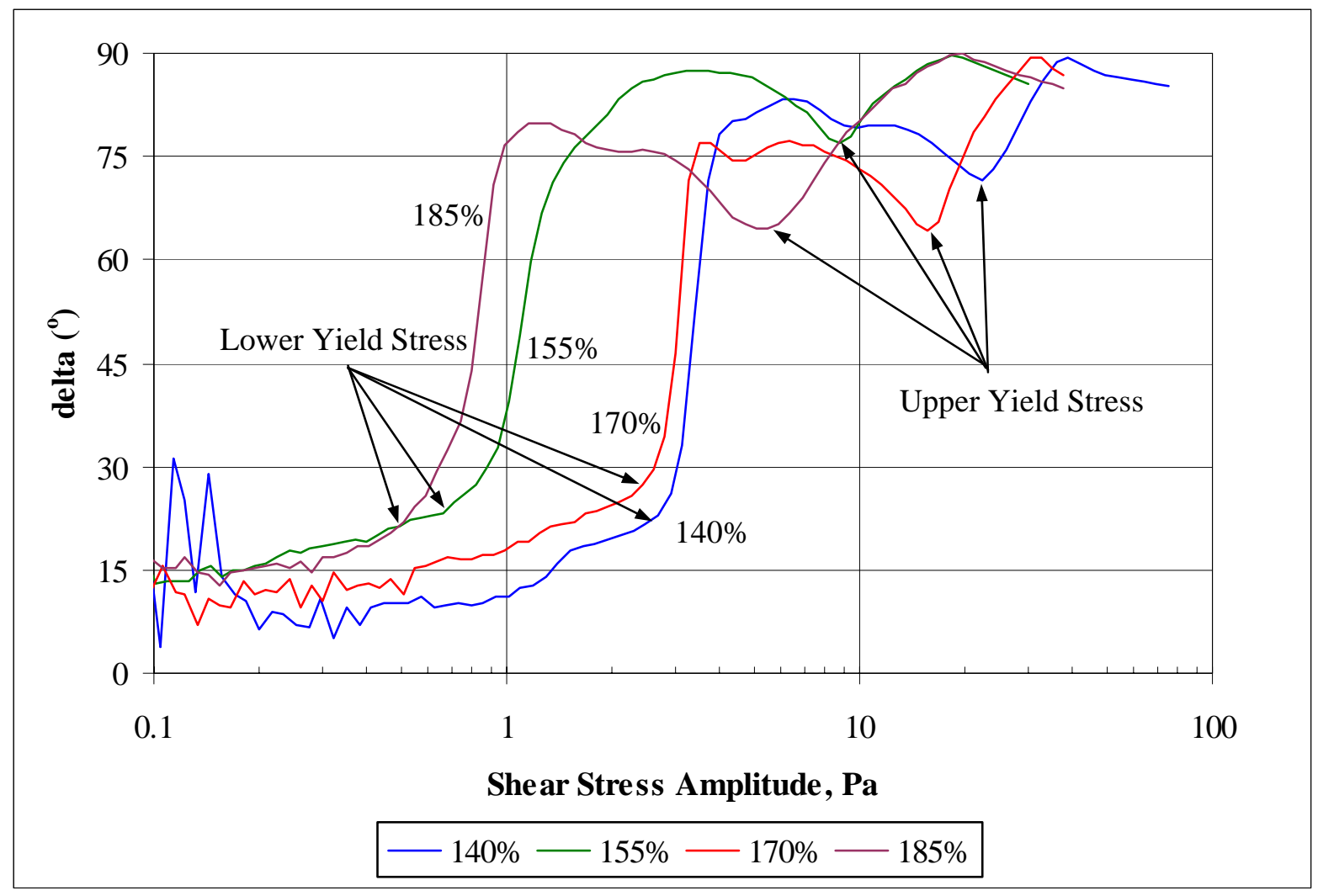

The relative ranking of the four samples is unchanged from the controlled shear stress flow curve test, Figure 27, and is the same at both the upper and lower yield stress transitions: $140 \%>170 \%>155 \%>$ $185 \%$. Note that on all four curves, once $\delta$ passed above $75^{\circ}$ for the first time, it then peaked and fell as stress continued to increase. This was quite common across the entire sample set. One interpretation is that the samples were rebuilding structure as $\delta$ fell. This hypothesis is supported by the fact that G' passes through a minimum and then rises. The alternative hypothesis is that the samples lost some liquidity. This does not seem to be supported, since G" was fairly constant over this stress range. It is unclear what sort of structure might have been forming. One possibility is that the particles assumed positions where it was easier for them to reorient themselves each time the flow direction reversed.

Plots of G” generally showed it to be decreasing with increasing shear stress. Plots of G' also showed a general trend of this type. The two trends taken together mean that the magnitude of the complex viscosity, $\left|\eta^{*}\right|$, decreased with increasing shear stress. This is the oscillation analog to pseudoplastic behavior, where the apparent viscosity decreases with increasing shear rate and shear stress. There was more structure to the G' data than expected, Figure 41. 
Figure 41. Storage Modulus of Four SB2/3 CETL SME Products

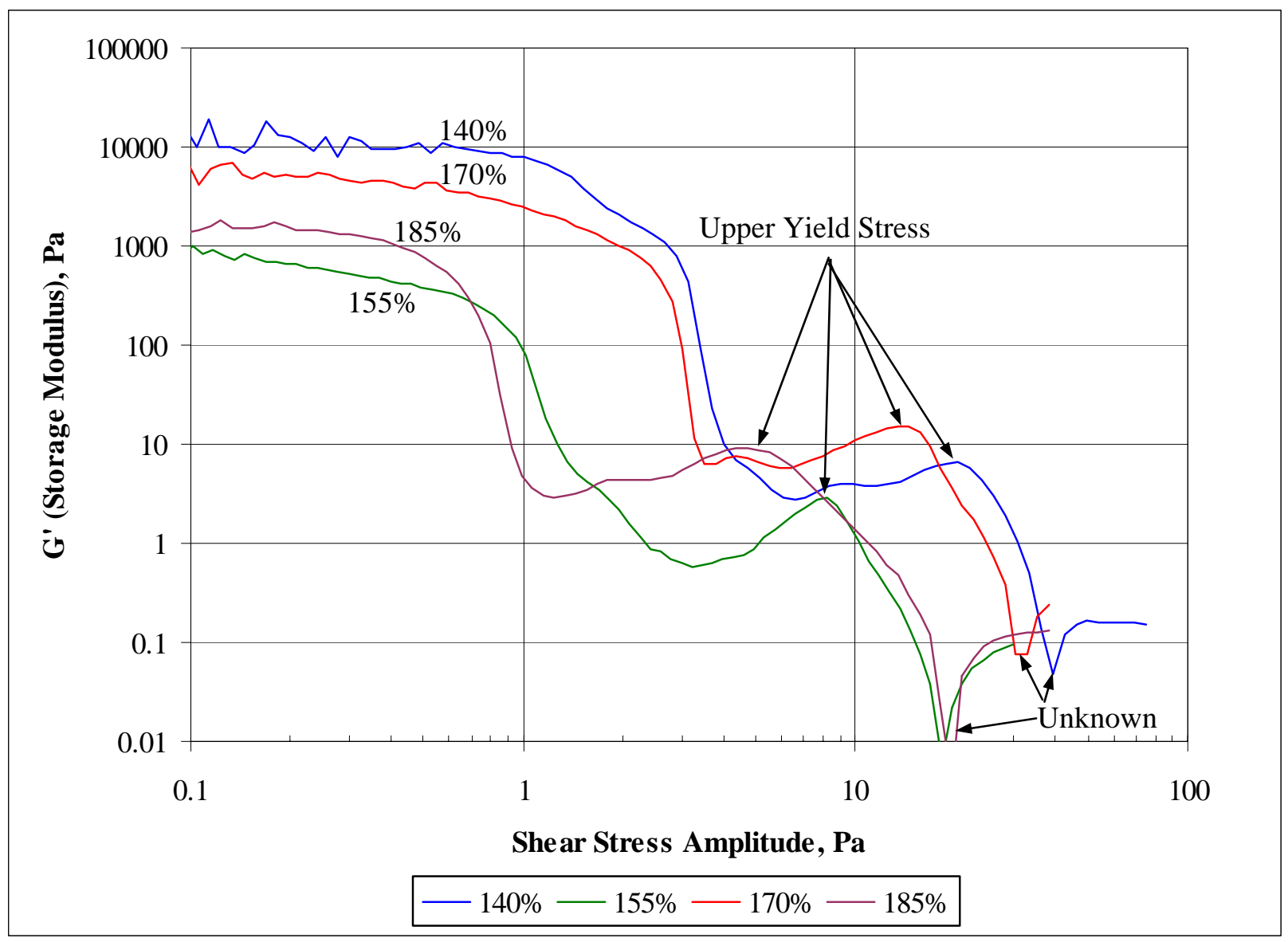

The storage modulus, G', generally went through a local minimum between the true yield stress and upper yield stress. It then proceeded to go through another local minimum at a stress above the upper yield stress. The significance of this has not been identified. One possibility is that the oscillatory flow may no longer be laminar (an oscillatory analog to Taylor vortices). Attention is drawn to this phenomenon here because it occurs in the stress region associated with flow, e.g. mixing, pipeline flow, pumping. It might warrant some further study.

Similar oscillation stress sweep measurements were made on both SRAT products and sludge simulants. Generally similar results were obtained in terms of the behavior of G', G”, $\delta$, and strain with respect to the applied stress.

Table 10 summarizes data on sludges and SRAT products obtained by both the oscillation stress sweep and by the controlled shear stress (CS) flow curve methods. True yield stresses and upper (Bingham) yield stresses are both shown. Values are all in Pa (multiply by ten to get values in dynes/ $\mathrm{cm}^{2}$ ). 
WSRC-TR-2004-00334

Revision 0

Table 10. Yield Stress Data, Pa, for Sludges and SRAT Products

\begin{tabular}{|c|c|c|c|c|}
\hline Sludges & CS-True & Oscillation-True & CS-Upper & Oscillation-Upper \\
\hline SB2 USC & 0.7 & 0.9 & 3.5 & 3.0 \\
\hline SB2 CETL & $0.4-1$ & 1 & 10 & $3.5-6$ \\
\hline Tank 8 USC & 0.34 & 0.35 & 2.3 & 1.5 \\
\hline SRAT Products & & & & 0.48 \\
\hline SB2 CETL & 0.11 & 0.13 & 0.5 & 3.1 \\
\hline $\begin{array}{c}\text { SB2 CETL } \\
\text { decanted }\end{array}$ & 0.67 & 0.35 & 4 & 2 \\
\hline SB2/3 CETL & Not seen & Not seen & 2 & \\
\hline
\end{tabular}

There were some samples that gave a fairly wide range for the true yield stress. SB2 CETL sludge gave a fairly wide range for the upper yield stress. This is the sludge that gives the hump in the controlled shear rate up ramp, so some sort of unusual behavior was not unexpected. The true yield stress values were generally similar between the two methods as seen with SME products. The upper yield stresses were generally less by the oscillation stress sweep than by the controlled stress ramp also as seen with SME products.

The SB2/3 SRAT product did not show a true yield stress by either method. The oscillation stress data shown in Figure 42 give the explanation.

Figure 42. Oscillation Stress Sweep on SB2/3 CETL SRAT Product

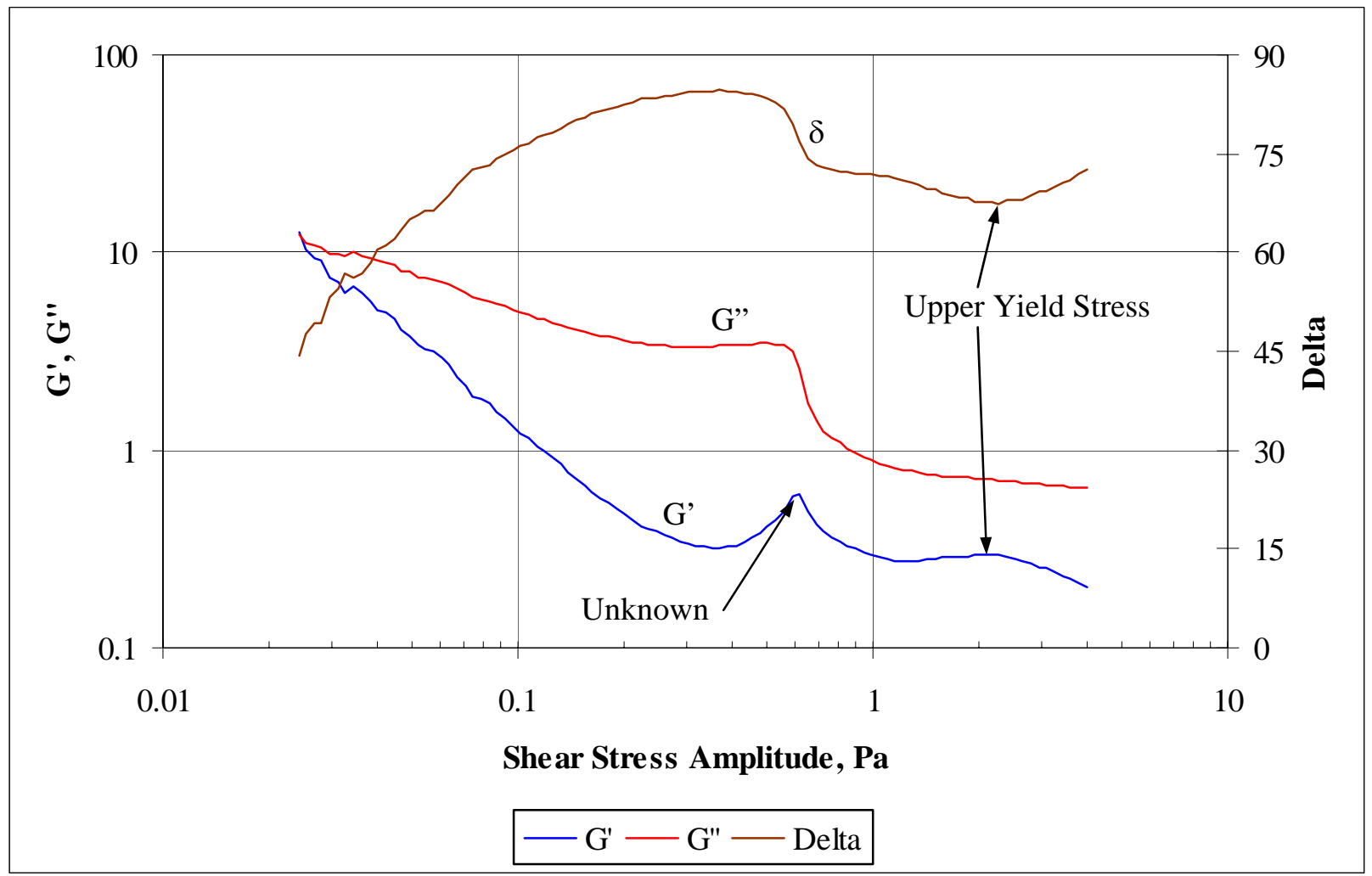

The data indicate that the sample was more liquid-like than solid-like from $0.02 \mathrm{~Pa}$ on up $(\delta>45$ degrees and G” > G'). From a practical standpoint, this sample has no true yield stress. In spite of this lack, the sample is clearly non-Newtonian and appears to follow a Bingham plastic model quite well above its 
upper yield stress, Figure 3. There was an unknown maximum in G' at about $0.7 \mathrm{~Pa}$. It was not associated with a change in the direction of $\delta$, and so has not been described as a yield stress. Yield stress was used only when the direction of change in $\delta$ with stress was positive (after being negative or constant).

\subsubsection{Effect of Frequency}

Most oscillation stress sweep data were taken at $1 \mathrm{~Hz}$. Some data were taken at other frequencies for comparison. These tests were done with the SB2/3 SME product, Run 6, at 155\% stoichiometry, made for Slurry Fed Melt Rate Furnace testing.

Figure 43. Effect of Frequency on SB2/3 CETL SME Product Oscillation Stress Sweeps

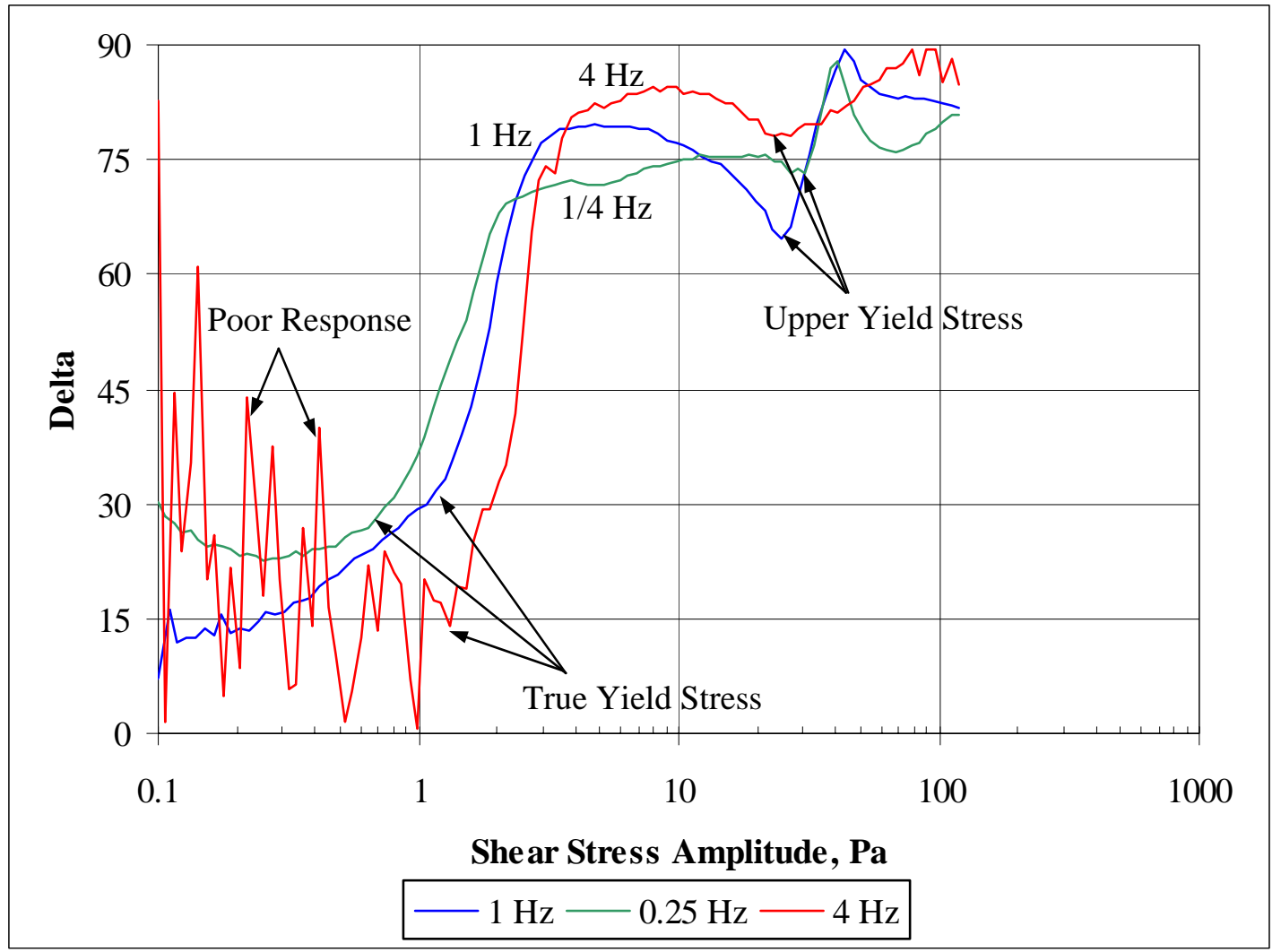

The data prior to the true yield stress were particularly noisy at $4 \mathrm{~Hz}$. The RheoWin software tracking the data did not show a deformation response that was wave-shaped until about $1 \mathrm{~Pa}$. This test took 400 seconds. The $1 \mathrm{~Hz}$ test took 800 seconds, and the $0.25 \mathrm{~Hz}$ test took 1600 seconds. All tests recorded 100 data points. These samples were not held for any consistent time prior to initiating the shear wave, which probably impacted the true yield point values. The shift toward higher values for the true yield stresses with increasing frequency was the one that ThermoHaake indicated would happen. The upper yield stress seemed less sensitive to the frequency choice.

Based on this test data, many of the subsequent oscillation stress sweep measurements were limited to 30 stress points, instead of 100 . This brought $1 \mathrm{~Hz}$ tests into the region of 4-6 minute durations. Tests at $0.25 \mathrm{~Hz}$ did not seem to offer any advantage and would take twice as long as $1 \mathrm{~Hz}$ tests. A $0.25 \mathrm{~Hz}$ choice might be needed if the primary region of study shifted below $0.1 \mathrm{~Pa}$. $1 \mathrm{~Hz}$ data became progressively noisier at lower shear stresses, and switching to $0.25 \mathrm{~Hz}$ or lower would tend to mitigate 
that. This was never necessary during the measurements made on the various sludges, SRAT products, and SME products in this study.

\subsubsection{Tackiness}

ThermoHaake has done some work with adhesives. They reported that if:

- $\tan \delta>1$, then a material flows, so it won't stay stuck to surfaces (flows off)

- if $\tan \delta \leq 0.3$ then a material is not sticky enough, i.e. too rigid/brittle (breaks off)

- if $0.3<\tan \delta<0.5$, then a material tended to be a good adhesive (tacky)

This tacky range translates to $16-27^{\circ}$ in the $\delta$ plots. The region from $0.5<\tan \delta<1$ was not explicitly discussed, but seems to equate to poorer adhesive properties. This range of $\delta$ typically occurs right above the true yield stress of nearly all of the slurry samples tested. There might be more information here that has not been fully developed. Some samples seemed to go through this range over a narrower range of stresses than others (perhaps due to the log scaling). It may be that the shear stress corresponding to $\delta \approx$ $20^{\circ}$ must be related to the typical shear stress produced by gravity on a vertical film in some manner to obtain a tacky slurry. This whole issue of the relationship of tackiness to $\delta$ could be explored further.

\subsubsection{Assessment of the Oscillation Stress Sweep Method}

The oscillation stress sweep is a good complement to the rotational tests. It confirmed the solid-like nature below the true yield stress. It confirmed the existence of two yield stress transitions in most of the samples tested. These are the true yield stress and an upper yield stress similar to the Bingham plastic yield stress in the rotational tests. The oscillation stress sweep upper yield stress was nearly always lower than the Bingham plastic yield stress, but this was not a disturbing finding. The nature of the flow above the true yield point is different in the oscillation measurement than in the rotational tests.

One point must be emphasized. Data obtained by this test are frequency dependent. If all of the tests had been done at $0.25 \mathrm{~Hz}$ or $4 \mathrm{~Hz}$ instead of $1 \mathrm{~Hz}$, then it would be expected that some of the yield stresses would shift higher or lower as a group than those reported in the tables. The data obtained at $1 \mathrm{~Hz}$ was consistent with rotational testing at the true yield stress.

The length of the test can vary depending on the range of stresses to be studied and the number of data points requested. Unlike the continuous ramp tests, where the number of data points requested has no impact on the ramp time, the oscillation stress sweep must make a dedicated measurement at each data point while doing nothing in between data points. A run with 30 data points is often $30 \%$ as long as a run with 100 data points (other settings such as half-wave option, wait periods, repeats, etc. being constant).

The individual rheometer bobs were calibrated for inertial effects. They were each given a unique label in the list of available bobs. This was recommended by ThermoHaake. Nothing in the data suggested that using one titanium Z38 bob with the inertial settings of a different titanium Z38 bob actually did anything to change the data obtained significantly. This may be due to the fact that the inertial settings were very close (the titanium bobs had very similar masses for a given size, e.g. Z38).

Pre-sheared samples were not entirely solid-like even following a ten minute hold in the instrument. They had $10^{\circ}<\delta<20^{\circ}$. Yielded samples were not entirely liquid-like. They had $70^{\circ}<\delta<90^{\circ}$. Samples generally had the loss, or viscous, modulus, G”, fall with increasing shear stress. Samples generally had the elastic modulus, G', fall with increasing shear stress until shortly past the true yield stress. The magnitude of the complex modulus decreased with increasing shear stress, indicating that the slurries were pseudoplastic (thinned with increasing shear). The same result was obtained by rotational 
methods. G' seemed to exhibit more structure than G”, Figure 41 and Figure 42. This was far more structure than expected from vendor examples. Example data from ThermoHaake, Figure 44, are given below for comparison.

Figure 44. Example Stress Sweep Data from ThermoHaake

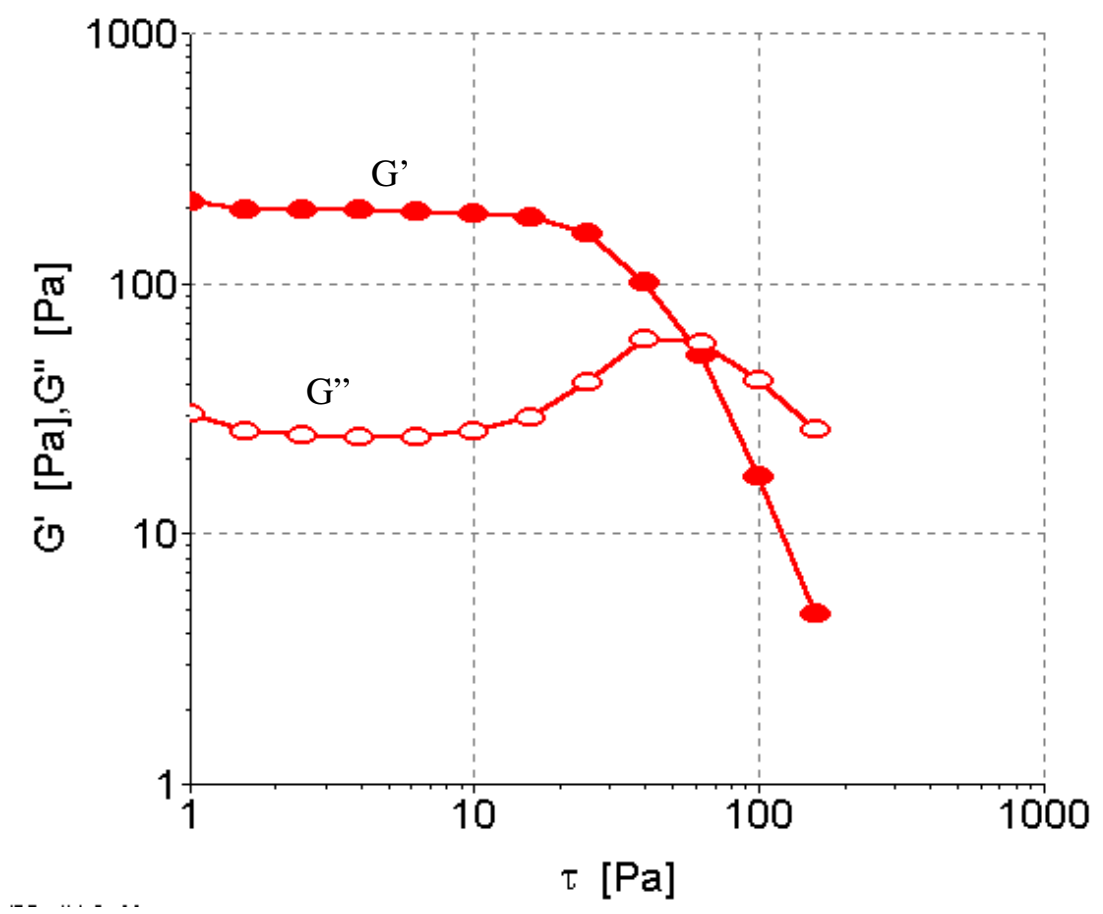

These data were from a gel whose structure broke down above about $15 \mathrm{~Pa}$. The data are similar prior to the true yield point to the DWPF slurry data, G' > G'. The large fall in G' as the sample yields is similar. The maximum in G" after yielding at 50-60 Pa was not seen in the DWPF slurries. G” often fell much like G' in this region in the DWPF samples. The number of data points being taken per decade of stress was fewer than used in this study. Conversely, there was apparently less behavior to be observed in the gel sample, presumably because it was a truly homogenous media unlike the slurry samples in this task. Finally, the example data does not continue to high enough stress to determine if other structures would have been present.

\subsubsection{Oscillation Frequency Sweep Measurements}

This test was used primarily to assess the suitability of $1 \mathrm{~Hz}$ testing in the oscillation stress sweep test. The test has other potential uses. A graph of the magnitude of the complex viscosity versus angular frequency is supposed to be nearly identical to the graph of the apparent viscosity versus shear rate obtained from rotational tests. Moving the angular frequency into the range of $300 \mathrm{rad} / \mathrm{s}$ to $600 \mathrm{rad} / \mathrm{s}$, however, did not appear to be feasible, but this is a reasonable range for the shear rate. Most data was obtained in the linear visco-elastic range, i.e. around the true yield point. The test can be used to investigate the response of material to impact using high frequencies or to gradual loads using low frequencies.

\subsubsection{Frequency Response of Viscosity Standard}

The response of the N35 viscosity standard was used to test the method before applying it to process samples. A series of tests were performed. The data were compiled into Figure 45. The nominal viscosity of $51.25 \mathrm{cP}(0.05125 \mathrm{~Pa}-\mathrm{s})$ is shown as a reference. 
WSRC-TR-2004-00334

Revision 0

Figure 45. NIST N35 Viscosity Standard Response to Oscillation Frequency Sweep

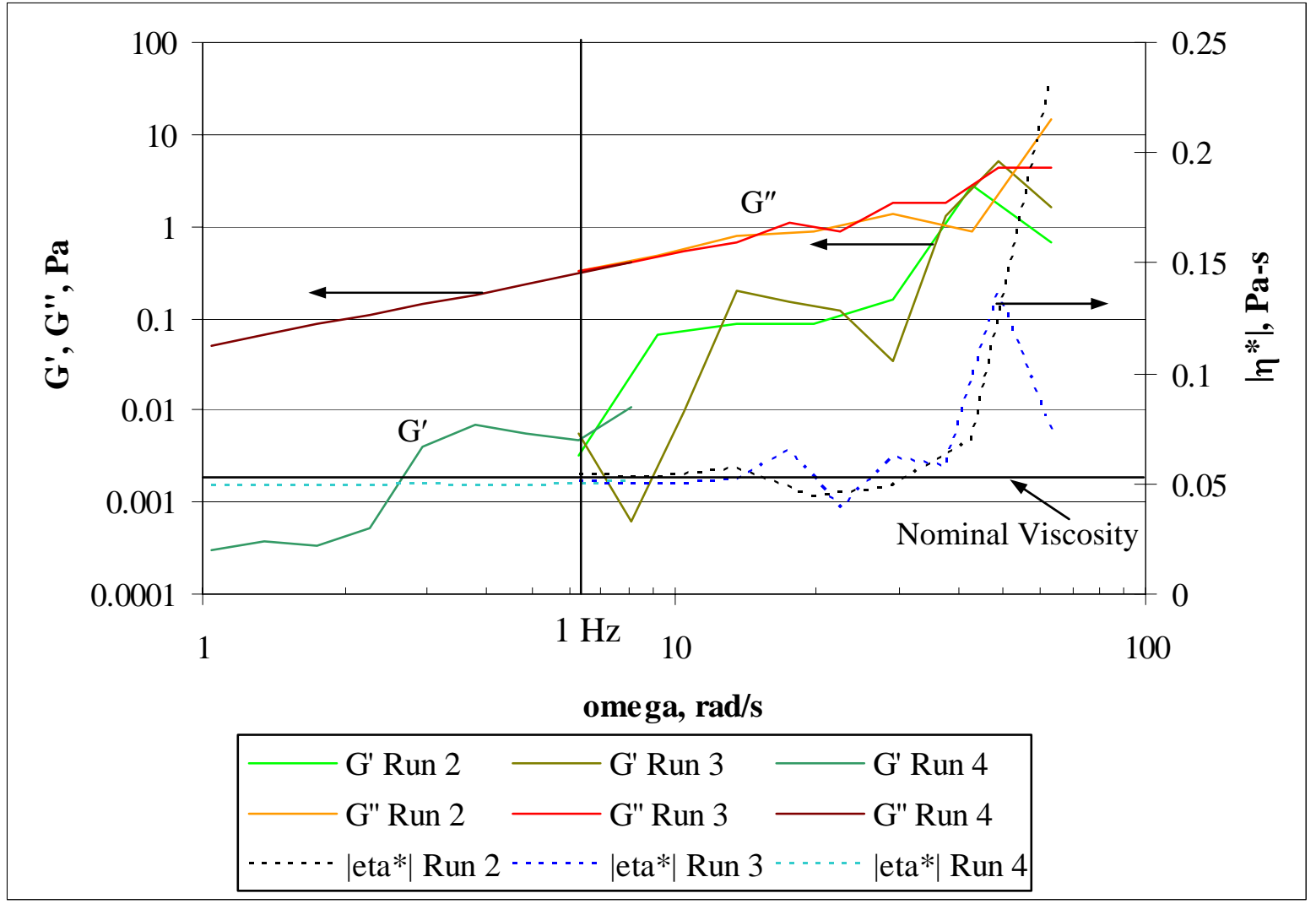

The viscosity standard behaved as expected up to about $40 \mathrm{rad} / \mathrm{s}(6 \mathrm{~Hz})$. The two moduli increased with increasing frequency, $\omega$, since $G^{*}=i \cdot \omega \cdot \eta^{*}$, and since the complex viscosity, $\eta^{*}$, is expected to be constant for all reasonably low frequencies. The vendor literature used the angular frequency in radians per second, $\omega$, for this test, instead of the rotational frequency, $\mathrm{f}$. The point where $\mathrm{f}=1 \mathrm{~Hz}$ corresponds to most of the data taken in the oscillation stress sweeps and oscillation time sweeps. This is equivalent to $\omega$ $=2 \pi$ radians/second. $1 \mathrm{~Hz}$ is clearly in a well-behaved region for N35 viscosity standard with the loss modulus almost two orders of magnitude larger than the storage modulus.

\subsubsection{Oscillation Frequency Sweeps and Process Samples}

Data for a slurry sample typically does not resemble that of the viscosity standard. This depends on the shear stress setting. This is usually chosen to be low, e.g. less than the true yield stress. An example of sludge simulant data is given in Figure 46. This sample was held for 10 minutes in the instrument before applying shear with the Z41 bob. The range in $\omega$ is one of the larger ones in this study. 
Figure 46. Oscillation Frequency Sweep on Tank 8 Simulant, 0.1 Pa, Z41

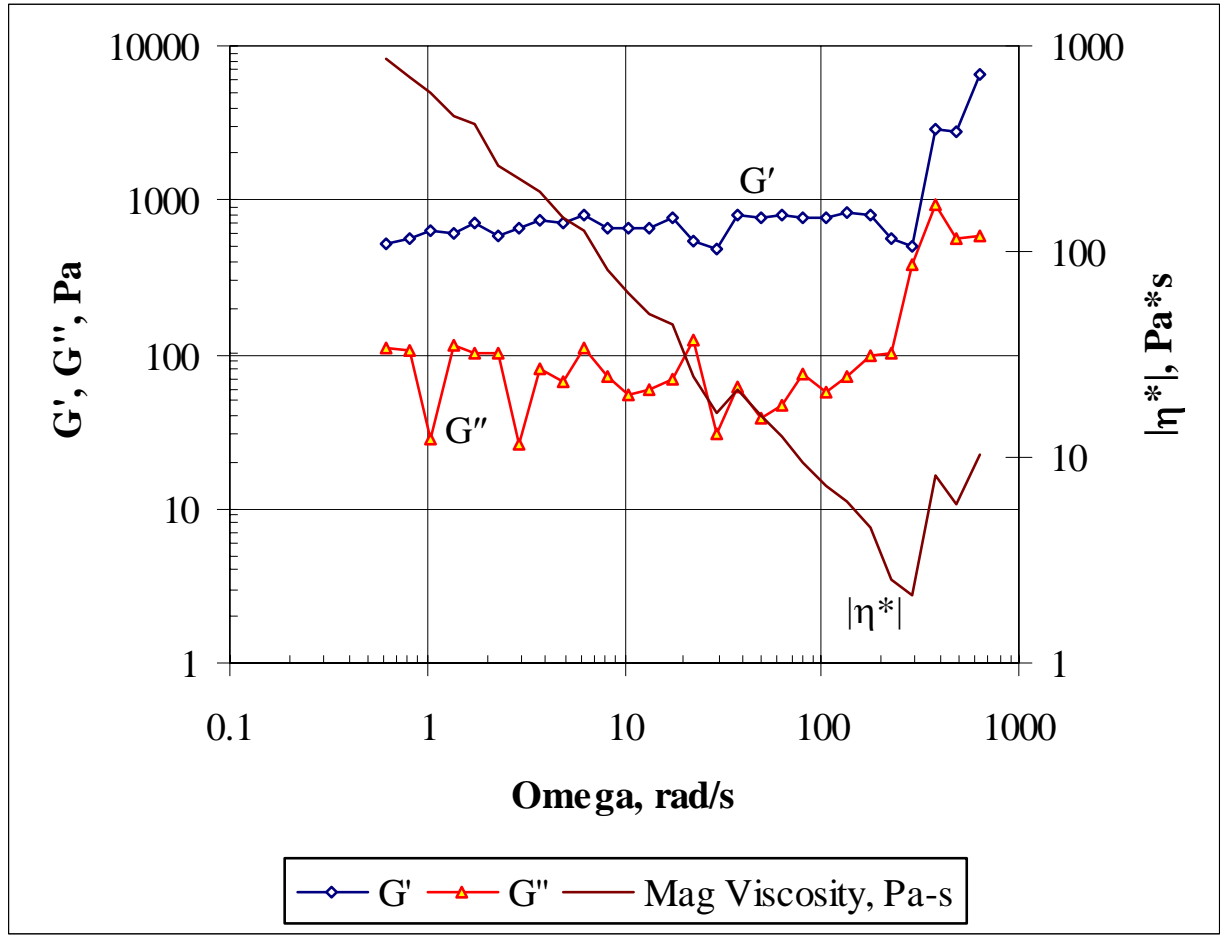

This measurement was made at a stress amplitude of $0.1 \mathrm{~Pa}$, while the true yield stress had been determined to be about $0.35 \mathrm{~Pa}$. Consequently, it was expected that the response would be solid-like. This was observed throughout the frequency spectrum, although G' $\approx G^{\prime \prime}$ at 300 radians/second (one data point) and both moduli had shifted upwards together. The magnitude of the complex viscosity fell continuously up to $300 \mathrm{rad} / \mathrm{s}$. The magnitude of the complex viscosity also spiked at about 300/s. Strain amplitudes confirm that the sample never yielded. The strain amplitude decreased with increasing frequency, i.e. as the bob oscillated faster, the arc that it traversed became smaller.

The Cox-Moore Rule, section 2.2.4.6, suggested that the viscosity should vary with shear rate in the same way that it does with oscillation frequency. This was clearly not the case for this sample, Figure 47. 
WSRC-TR-2004-00334

Revision 0

Figure 47. Test of Cox-Moore Rule on Tank 8 Simulant

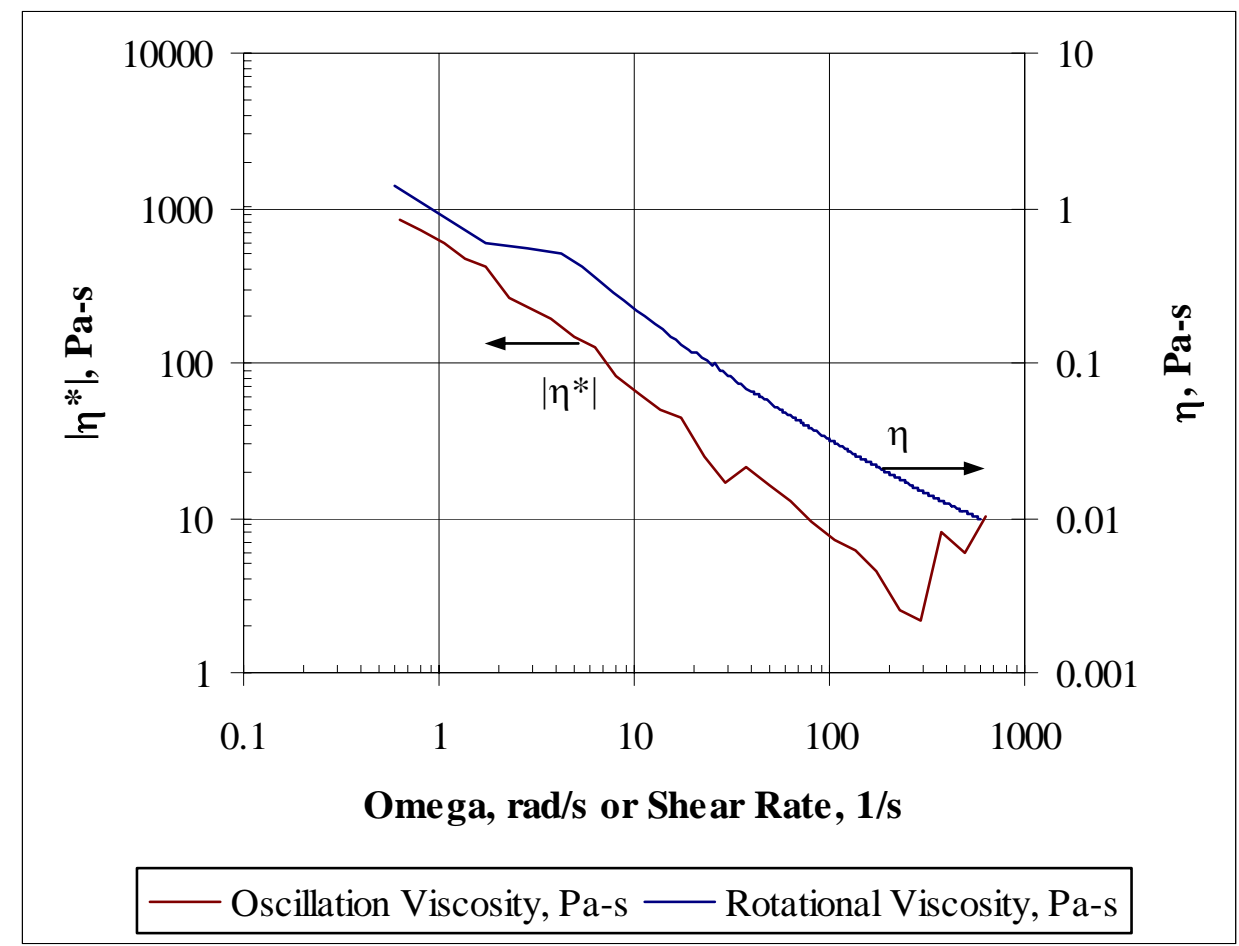

The two viscosities differ by almost three orders of magnitude. This is most likely due to testing at a shear stress below the true yield stress in the oscillation frequency sweep compared to testing at much higher shear stresses in the controlled shear rate flow curve. This limitation needed to be established experimentally, however, since the shear rate and frequency ranges overlapped appropriately. The rotational data is from a controlled shear rate flow curve up ramp to 600/s shear rate. Both measurements used the Z41 bob and had a long hold time in the instrument before applying shear. The average slopes of the two curves would be similar, and this might relate to a material property. (The slope would be related to the exponent in the power law rheological equation in the case of the controlled shear rate data.)

The next graph is for SB2/3 SRAT product, Figure 48. This sweep was at $0.5 \mathrm{~Pa}$, while no true yield stress was found down to $0.02 \mathrm{~Pa}$. The Bingham plastic yield stress was about $2 \mathrm{~Pa}$. This sweep was still in the region below the Bingham plastic yield stress. This sweep also covered a wide range of frequencies. 
Figure 48. Oscillation Frequency Sweep on SB2/3 SRAT Product at 0.5 Pa

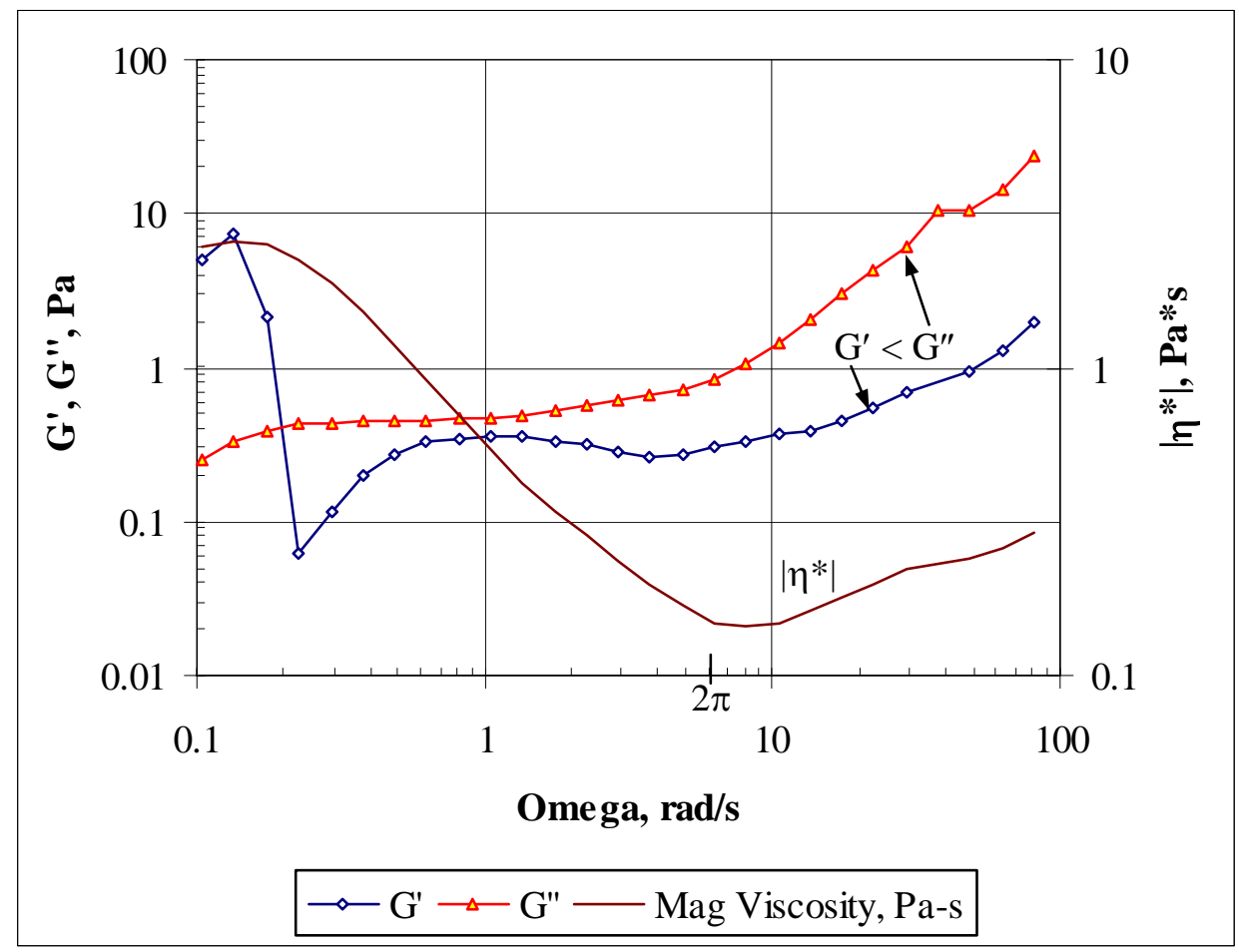

The frequency range is dominated by a region where G” > G', i.e. the sample is mostly liquid-like in this test. This is true for a wide range about $2 \pi \mathrm{rad} / \mathrm{s}$, or $1 \mathrm{~Hz}$. This indicates that $1 \mathrm{~Hz}$ is a robust frequency for other testing. Rigidity at low frequencies was not expected, and is not trustworthy. The sample had the largest deformations at low frequency. The data near $0.1 \mathrm{rad} / \mathrm{s}$ are probably a settling-in period for the rheometer, rather than a solid-like zone for the sample.

The Cox-Moore Rule failed on this sample result as well, perhaps because the oscillation stress was still below the Bingham plastic yield stress of the sample. The rotational apparent viscosity was around 0.020.4 Pa-s for shear rates from 100/s down to 1/s. These were about one order of magnitude lower than the oscillation-based viscosity, however, rather than three orders for the sample below the true yield stress.

\subsubsection{Assessment of Oscillation Frequency Sweep}

This measurement is fairly straight-forward, but benefits from an adequate hold time prior to initiating shear. Ten minute or longer hold times are recommended for tests at a stress below the true yield stress. There were no big surprises in the data. This method did not offer as much useful data as the oscillation stress sweep on a per measurement basis. A frequency sweep range of $0.5-50 \mathrm{rad} / \mathrm{s}$ is generally suitable for assessing whether or not to use a $1 \mathrm{~Hz}$ setting in the oscillation stress and time sweep measurements.

\subsubsection{Oscillation Time Sweep Measurements}

Oscillation time sweeps are performed at a specified amplitude of the shear stress. The frequency is also specified. The standard frequency for these tests was $1 \mathrm{~Hz}$. The two moduli, G' and G”, along with $\delta$ and $\left|\eta^{*}\right|$, are monitored as a function of time. The primary variable of interest is $\left|\eta^{*}\right|$. If $\left|\eta^{*}\right|$ falls with time, then the sample should be acting in a thixotropic manner. If $\left|\eta^{*}\right|$ rises with time, then the sample should be acting in a rheopectic manner. 
The advantage of this oscillation test over the constant shear rate time curve rotational test is that the stress can be set below the true yield stress, between the true and upper yield stress, or above the upper yield stress. The constant shear rate selected in the time curve is typically one that will force the sample to shear stresses above the yield stress (it would take an extremely low shear rate to not deform the sample enough over 15 minutes to cause it to yield and flow). Therefore, this oscillation test can probe two regions of sample behavior that are not readily accessible by the rotational constant shear rate test. This strategy was taken on a number of DWPF simulant slurry samples. No test was made with the viscosity standard, since the other two oscillation tests indicated that little of interest would be seen.

\subsubsection{Oscillation Time Sweeps of Process Samples}

SB2 CETL sludge (hump) was tested at four stresses. These are given in Figure 49 through Figure 52. They will serve to illustrate the features of interest. SB2 CETL sludge measurements were made with the Z41 bobs. An instrument malfunction aborted the $6 \mathrm{~Pa}$ test. The other three tests had a ten minute duration. The true yield stress was 0.4-1 $\mathrm{Pa}$ and the upper (Bingham-like) yield stress was 3.5-10 Pa based on the previous tests discussed above. 
Figure 49. 0.1 Pa Oscillation Time Sweep of SB2 CETL Sludge at $1 \mathrm{~Hz}$.

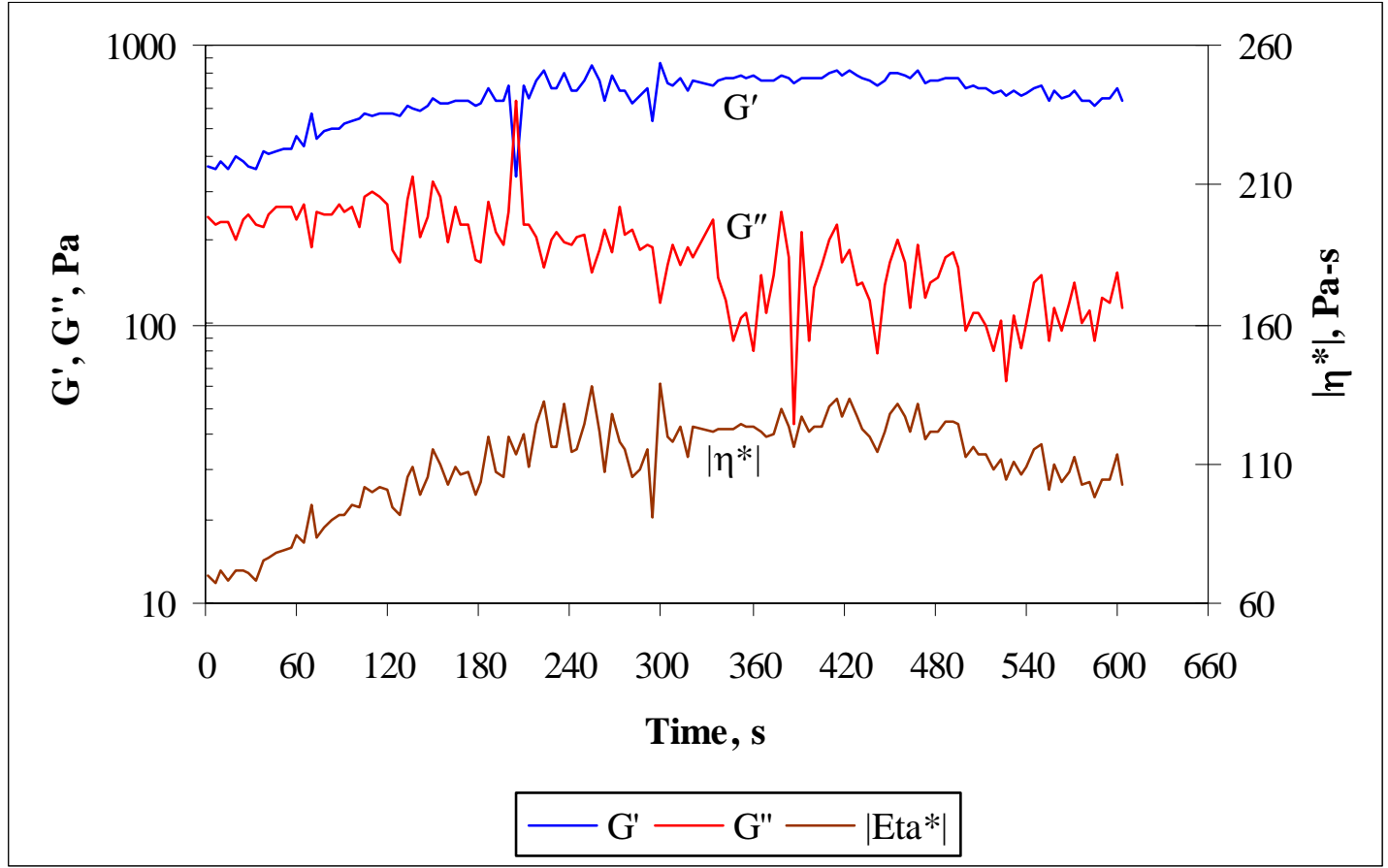

Figure 50. 1.5 Pa Oscillation Time Sweep of SB2 CETL Sludge at $1 \mathrm{~Hz}$.

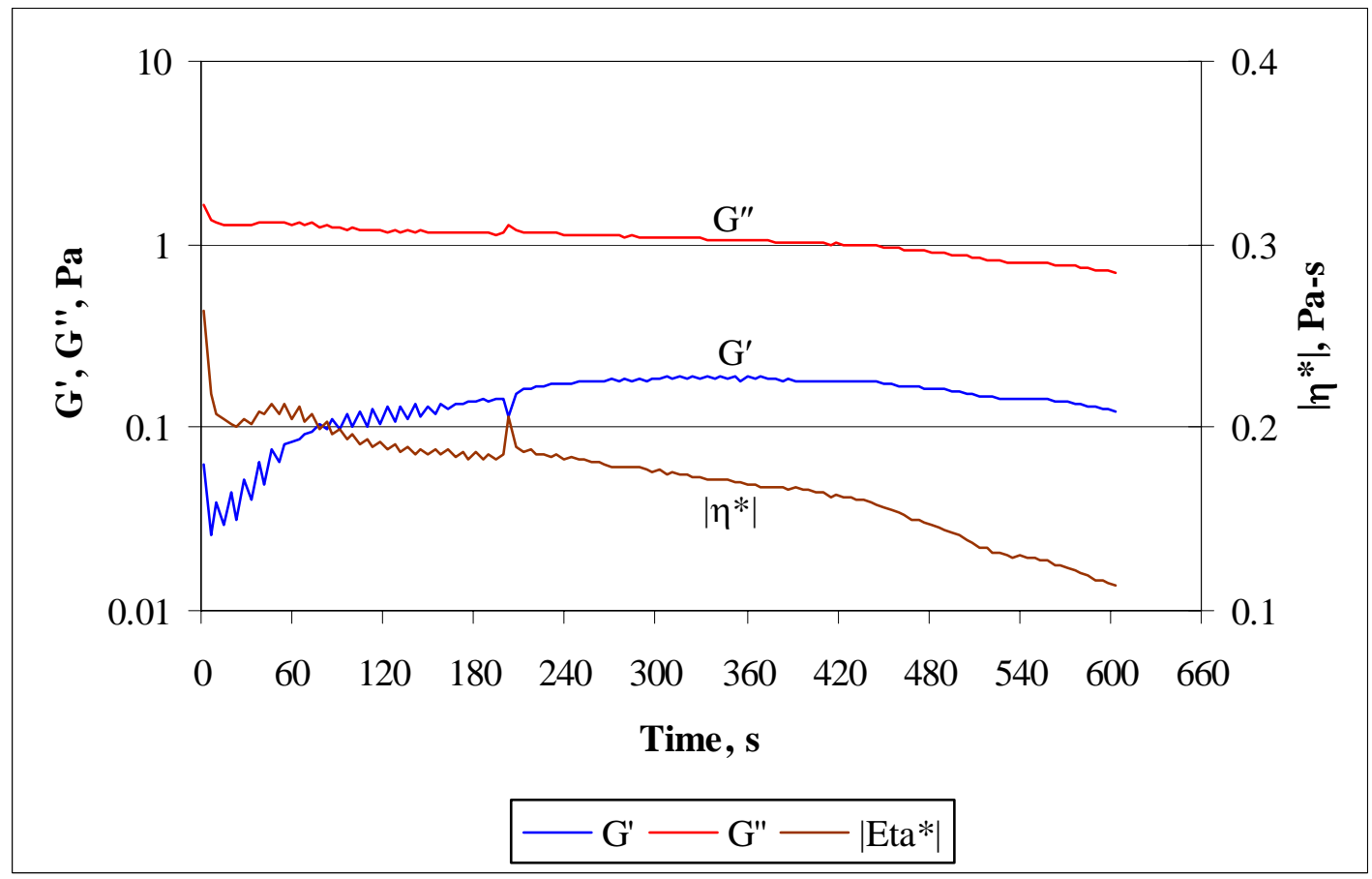


Figure 51. 6 Pa Oscillation Time Sweep of SB2 CETL Sludge at $1 \mathrm{~Hz}$.

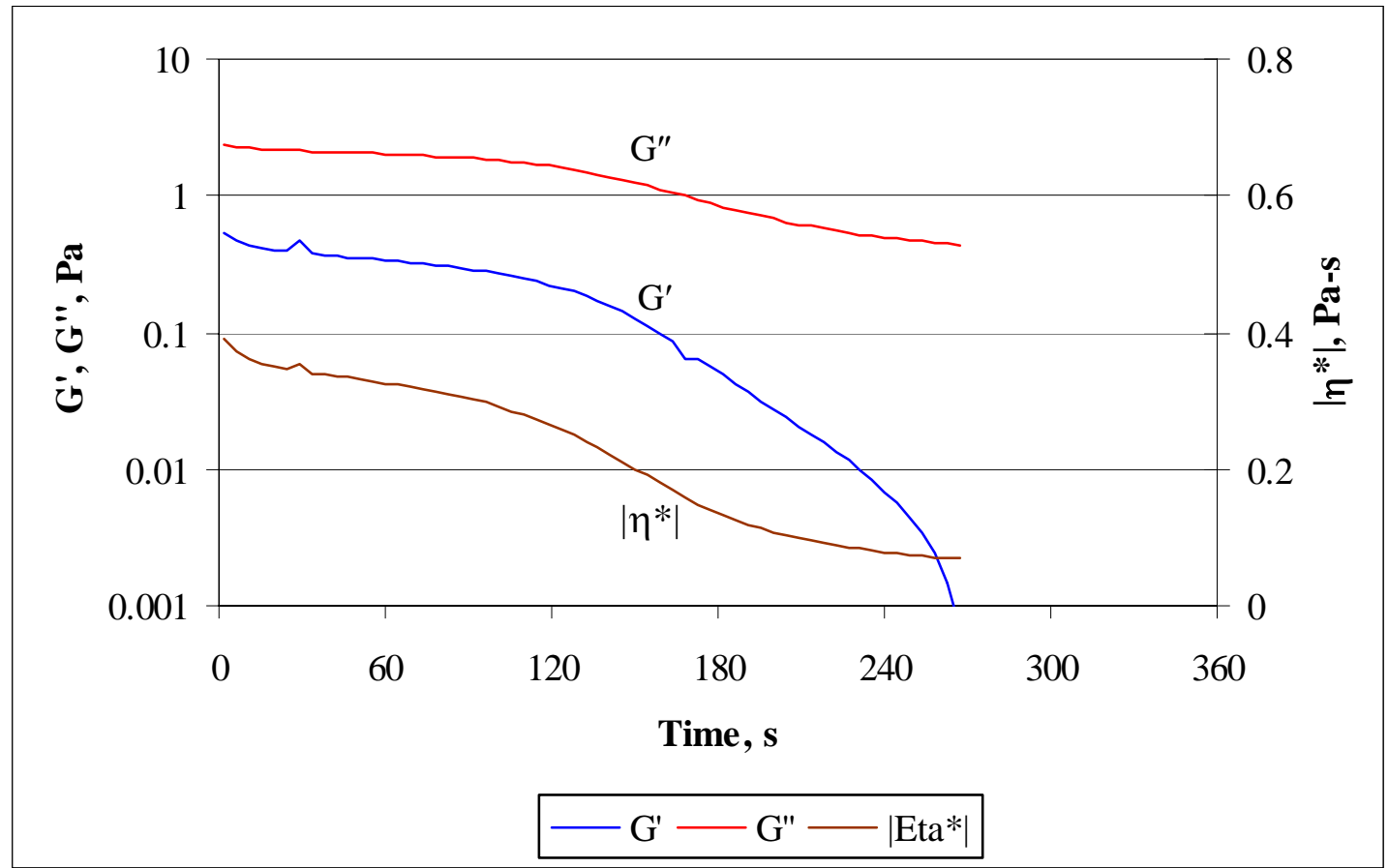

Figure 52. 15 Pa Oscillation Time Sweep of SB2 CETL Sludge at $1 \mathrm{~Hz}$.

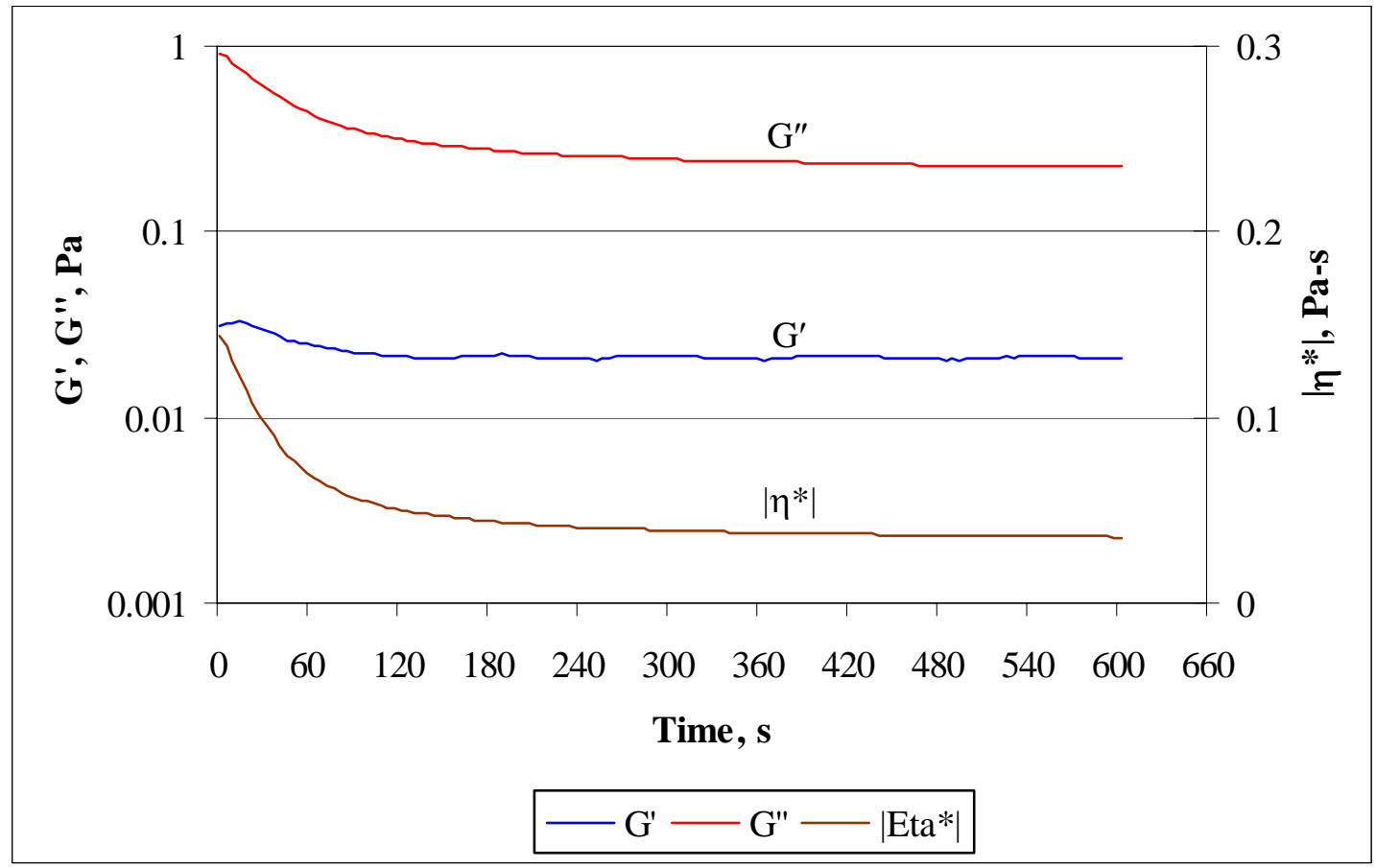

The 0.1 Pa measurement was expected to be below the true yield stress. Figure 49 indicates that G' > G' for the entire ten minutes. $\delta \approx 10^{\circ} .\left|\eta^{*}\right|$ increased for about three minutes, seemed to stabilize, and then appeared to fall slightly. The data was the noisiest of the four sets presumably because the oscillation deformation amplitude was the smallest (most difficult to measure). 
The 1.5 Pa measurement was not noisy. G” was greater than G' for the entire test. The sample was above the true yield stress by this measure. $\left|\eta^{*}\right|$ fell during most of the test. The sample acted in a thixotropic manner by thinning with time under shear. The aborted $6 \mathrm{~Pa}$ measurement was similar to the 1.5 measurement except that G' was even lower. It is possible that a loss of the G' element caused this test to abort.

The $15 \mathrm{~Pa}$ measurement was above both the true and the Bingham plastic yield stresses by other methods. The sample thinned rapidly for about three minutes, and then continued thinning very slowly for the last seven minutes. This generally indicates thixotropy.

The data from these tests and the constant shear rate time curve were compared in a summary table, Table 11. The measure of thixotropy selected for this table was the ratio of the viscosity at the end of the test relative to the viscosity after two minutes into the test, $\eta_{10} / \eta_{2}$. The oscillation test ratios were based on the magnitude of the complex viscosity. The apparent viscosity, $\eta$, was used for the rotational test. A neutral time dependence denotes a condition between mildly rheopectic and mildly thixotropic behavior. Typical viscosities are given just to give an idea of the order of magnitude of the viscosity in each of the test regions. The state is based on G' versus G” data at $1 \mathrm{~Hz}$.

Table 11. Time Dependent Nature of SB2 CETL Sludge

\begin{tabular}{|l|c|c|c|c|}
\hline Test & State & Typical $|\eta *|, \eta$ & $\eta_{10} / \eta_{2}$ & Time dependence \\
\hline $0.1 \mathrm{~Pa}$ Oscillation & Solid-like & $110,000 \mathrm{cP}$ & 1.02 & Neutral \\
\hline $1.5 \mathrm{~Pa}$ Oscillation & Between yield stresses & $164 \mathrm{cP}$ & 0.60 & Thixotropic \\
\hline $15 \mathrm{~Pa}$ Oscillation & Liquid-like & $36 \mathrm{cP}$ & 0.69 & Thixotropic \\
\hline 200/s Rotational & Liquid-like & $34 \mathrm{cP}$ & 0.80 & Thixotropic \\
\hline
\end{tabular}

Rotational and oscillation data in the liquid-like region were consistent in identifying SB2/3 sludge simulant as significantly thixotropic. The $15 \mathrm{~Pa}$ oscillation sweep indicates even more thixotropy than the constant shear rate time curve. Thixotropy was also indicated in the controlled shear rate flow curve measurement, but it was harder to quantify because of the hump. This much time dependence indicates that this sample is not a good candidate for the Bingham plastic fluid model.

SB2/3 data can be compared to USC Tank 8 simulant data, Table 12.

Table 12. Time Dependent Nature of USC Tank 8 Sludge

\begin{tabular}{|l|c|c|c|c|}
\hline Test & State & Typical $|\eta *|, \eta$ & $\eta_{10} / \eta_{2}$ & Time dependence \\
\hline 0.1 Pa Oscillation & Solid-like & $99,000 \mathrm{cP}$ & 1.20 & (nearly neutral) \\
\hline 0.7 Pa Oscillation & Between yield stresses & $1000 \mathrm{cP}$ & 1.0 & Neutral \\
\hline 4 Pa Oscillation & Liquid-like & $24 \mathrm{cP}$ & 1.04 & Neutral \\
\hline 200/s Rotational & Liquid-like & $19 \mathrm{cP}$ & 0.99 & Neutral \\
\hline
\end{tabular}

The time dependence did not stabilize to a consistent trend in the solid-like range at $0.1 \mathrm{~Pa}$. The ratio of ten minute to five minute viscosity, $\eta_{10} / \eta_{5}$, was 0.9 . This indicated that the apparent rheopexy seen by $\eta_{10} / \eta_{2}$ was transient. Otherwise, the USC Tank 8 simulant was virtually time independent, i.e. a good candidate for the Bingham plastic fluid model. This sludge tested very differently from SB2 CETL sludge. It seemed to be much more like real waste sludge in its lack of time dependent rheological properties. 


\subsubsection{Assessment of Oscillation Time Sweep}

This test was not difficult to perform. Samples were best left free of shear for at least ten minutes for stress settings below the upper yield stress. The test might be enhanced by extending it from ten minutes to fifteen minutes. Some of the constant shear rate time data indicated that some samples settled down to more constant properties in the additional five minute period. This might apply to this test as well.

The general usefulness of this test is expected to be somewhat limited. Its primary use seems to be as a probe of time dependence at various shear stress levels. This information can be compared between different samples to see if the time dependent features are changing. At this time, information of this type is not available from the two radioactive rheometers in service (RV20 and RV30). Therefore, radioactive benchmark data cannot be obtained at this time for comparison to simulant data. 


\subsection{General Rheological Measurement Approach}

The acquisition of rheological data is such that it is often an interactive process between the researcher, the instrument, and the sample. This is particularly true when a sample exhibits some form of unusual behavior such that the expected shape of a normal flow curve is not produced. The simple two step process of instrument check-out with viscosity standard followed by sample measurements using a controlled shear rate flow curve measurement potentially obtains results that are inadequate to accomplish the goals of the customer. The most typical goals are taken to mean producing the Bingham plastic yield stress and consistency for a sample for comparison either with other samples or with the DWPF design bases. The attached flowchart, Figure 53, lays out a reasonable logic path for dealing with DWPF slurry samples. Additional details on the individual method protocols are given in Koopman (2004b). The rheometer method protocols themselves, however, were developed from the measurement method development work described in this report.

The protocol flowchart lays out a sequence of steps and decisions that the researcher must make when performing a rheological characterization. More specialized protocols were also generated for many of the individual steps on the flowchart. These protocols are specific to the measurement or task being performed. The use of multi-ramp flow curves given in Figure 53 in the fifth block down, for example, was discussed in section 3.1.4. 
Figure 53. Sample Rheological Characterization Flowchart

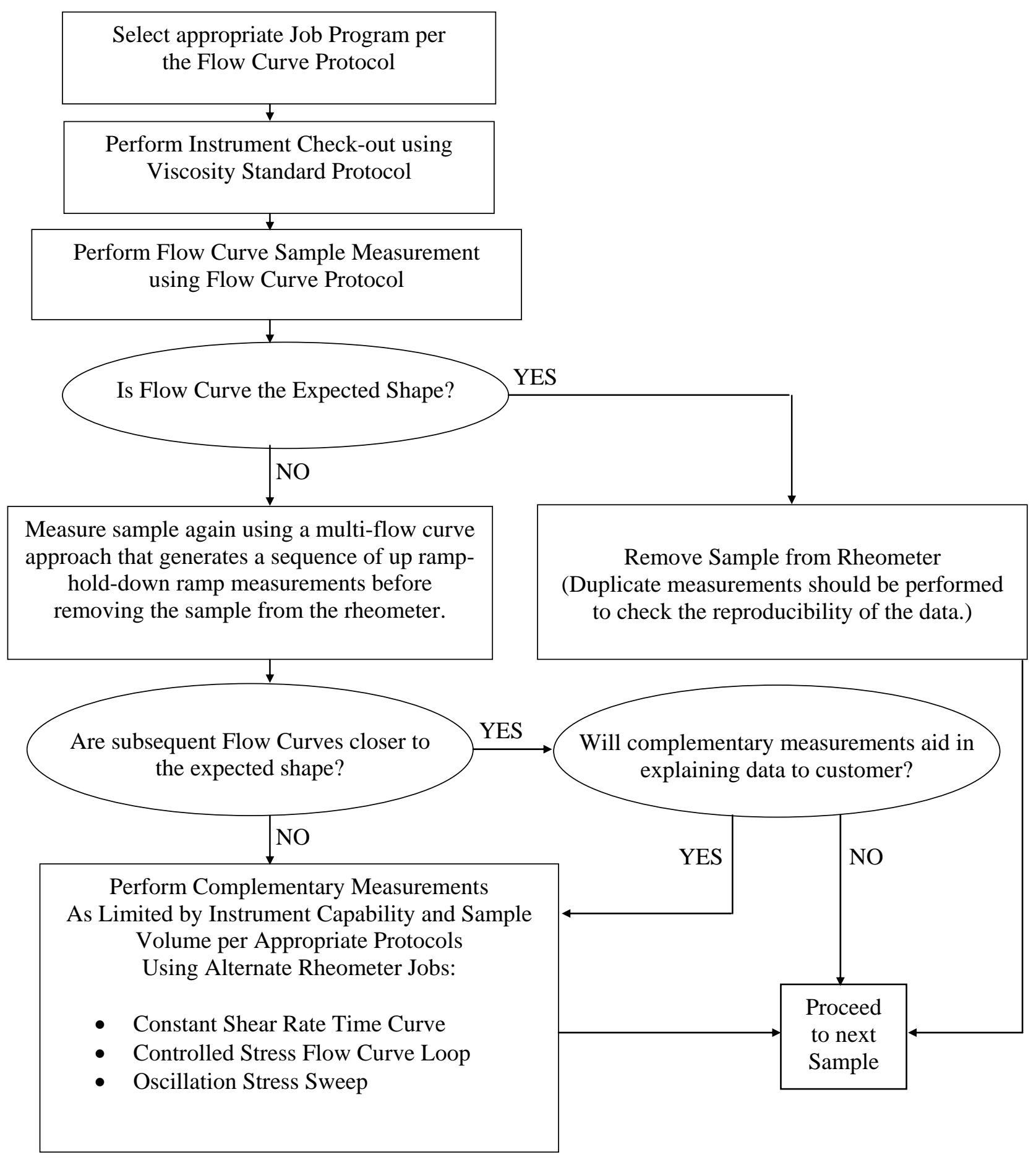


WSRC-TR-2004-00334

Revision 0

This page intentionally left blank. 


\subsection{CONCLUSIONS}

A set of goals was laid out in the Introduction, section 1.0, of this report. Progress related to two of these goals is summarized here. Variations in the rheological behavior of simulant DWPF slurry samples were also investigated and are documented in Koopman (2005). The number of rheological methods applicable to DWPF samples has been increased to include:

- Controlled shear stress flow curve

- Constant shear rate time curve

- Small deformation yield point

- Creep-recovery analysis

- Oscillation stress sweep

- Oscillation frequency sweep

- Oscillation time sweep

The controlled shear rate flow curve measurement gives a reproducible flow curve for a time independent sample and one measure of thixotropy for a time dependent sample. The supplemental measurements listed above provide information beyond that obtained from the controlled shear rate flow curve, i.e. provide more than just data that might be converted into a Bingham plastic yield stress and consistency. The following method specific paragraphs provide elaboration.

The leading candidate for full implementation as a supporting test to the controlled shear rate flow curve is the constant shear rate time curve. This test can be performed by all four rheometers currently in service for DWPF slurry samples, both simulant and radioactive. This test gives a clear, quantifiable measure of the time dependence of the rheological properties of a sample. This is a better test than the controlled shear rate flow curve for time dependent samples, because it is possible to monitor the time dependence continuously under constant, controlled conditions.

The controlled shear stress flow curve and the oscillation stress sweep are similar in many respects. They both provide insight into the behavior of slurries at low shear stresses that are not well-characterized by the controlled shear rate flow curve. These two tests both revealed the existence of two yield transitions in most of the slurry samples. The lower transition is a solid-like to liquid-like transition taken to denote the true yield stress of the sample. The upper transition is marked by a change in behavior associated with a more fully developed flow pattern. This upper transition corresponds fairly closely with the traditional Bingham plastic yield stress. It can vary from it somewhat numerically depending on the analytical method used to extract it from the data. The controlled shear stress flow curve can produce a graph that contains the same information as the controlled shear rate flow curve. A direct comparison between a pair of measurements can indicate potential measurement issues. The oscillation stress sweep produces information on the elastic and loss moduli, which provide a direct assessment of the solid-like and liquid-like character of the sample. This information is only obtainable through oscillation measurements.

Detailed recommendations for performing the controlled shear rate flow curve, constant shear rate flow time curve, controlled shear stress flow curve, and oscillation stress sweep were formalized and issued as a rheology protocol for DWPF slurries, Koopman (2004b). The remaining new tests, summarized in the next two paragraphs, were not incorporated into the new protocols. They did not appear to be the optimal tests for the available resources and current set of issues. 
The small deformation yield point and the creep-recovery analysis were more difficult tests to implement. They did not provide information that could not be obtained by the other tests. Even when performed properly, they are only intended to find the true yield stress. Consequently, they were not incorporated into the list of follow-up measurements in the new recommended protocols for making rheology measurements of DWPF samples.

The oscillation frequency sweep was primarily useful in providing a check on the robustness of the frequency selected for the oscillation stress and time sweeps. The oscillation time sweep was a test that could probe a sample's characteristics as a function of time at various stresses. This capability is not readily available from the controlled stress and controlled shear rate direct rotational methods. 


\subsection{RECOMMENDATIONS/PATH FORWARD}

The findings in this study were used to generate a set of guidelines, or protocols, for performing a characterization of a DWPF slurry sample. Characterization begins with the controlled shear rate flow curve. Testing can expand to include the controlled shear stress flow curve, constant shear rate time curve, and oscillation stress sweep if the information desired warrants the additional work. Signs of time dependence in the controlled shear rate flow curve are justification for making a multiple flow curve measurement where the up and down shear rate ramps are repeated two or three times.

A parallel document, Koopman (2004b), contains these protocols in the form of guidelines for performing these measurements. The guidelines were based on the method development that is summarized in this report. It is recommended that those guidelines be implemented on future radioactive and simulant DWPF slurry measurements.

DWPF reported that SB1B slurries seemed to be "tackier" than SB1A slurries, i.e. they were more difficult to decontaminate from the standpoint of rinsing the slurry off of surfaces. The tackiness of slurries may well be linked to the true yield stress and the term $\delta=\operatorname{Arctan}(G " / G$ ”), both described in this report for the first time with respect to DWPF simulants. Some rheological modeling work could be undertaken to develop governing equations that relate gravity flow, film thickness, and yield stress before undertaking any future experimental work in this area. 


\subsection{REFERENCES}

Alderman, N. J., and N. I. Heywood, “Improving Slurry Viscosity and Flow Curve Measurements”, CEP 4, p. 27 (2004a).

Alderman, N. J., and N. I. Heywood, “Making Accurate Slurry Flow Curve Measurements”, Chemical Engineering Progress 5, p. 35 (2004b).

Baich, M. A., C. C. Herman, D. C. Koopman, D. R. Best, T. K. Snyder, and M. F. Williams, Processing Options and Impact of Incorporation of ARP in the DWPF Process Flow Sheet (U). WSRC-TR-200300326, Savannah River Site, Aiken, SC 29808 (2003).

Baich, M. A., Sludge Batch 2-3 Blend Flowsheet Simulations: Determination of Acid Addition Window. WSRC-TR-2004-00042, Savannah River Site, Aiken, SC 29808 (2004a).

Baich, M. A., Sludge Batch 2-3 Blend Flowsheet Simulations: Process Variability Study. WSRC-TR2004-00225, Savannah River Site, Aiken, SC 29808 (2004b).

Bannochie, C. J., D. C. Koopman, and D. T. Herman, Impact of Uranium Levels on Sludge Batch 2 Simulant Processing. WSRC-TR-2004-00206, Savannah River Site, Aiken, SC 29808 (2004).

Herman, C. C., D. C. Koopman, D. R. Best, T. K. Snyder, M. F. Williams, Sludge Batch 3 Simulant Flowsheet Studies: Final Phase SRAT/SME Results. WSRC-TR-2003-00422, Savannah River Site, Aiken, SC 29808 (2003).

Koopman, D. C., Sludge Batch 2 (Macrobatch 3) Flowsheet Studies with Simulants (U). WSRC-TR2000-00398, Savannah River Site, Aiken, SC 29808 (2000).

Koopman, D. C., Summary of Results from the Preparation of Nominal Tank 8/Tank40 Melter Feed in the 1/240 ${ }^{\text {th }}$ Glass Feed Preparation System (U). SRT-GPD-2001-020, Savannah River Site, Aiken, SC 29808 (2001).

Koopman, D. C., A Comparison of Rheology Data for Radioactive and Simulant Savannah River Site Waste (U). WSRC-TR-2004-00044, Savannah River Site, Aiken, SC 29808 (2004a).

Koopman, D. C., Rheology Protocols for DWPF Samples. WSRC-RP-2004-00470, Savannah River Site, Aiken, SC 29808 (2004b).

Koopman, D. C., Rheological Characterization of Unusual DWPF Slurry Samples (U). WSRC-TR-200400519, Savannah River Site, Aiken, SC 29808 (2005).

Smith, G. L. and K. Prindiville, Guidelines for Performing Chemical, Physical, and Rheological Properties Measurements. 24590-WTP-GPG-RTD-001, Revision 0, (2002).

Stone, M. E. and P. L. McGrier, Impact of DWPF Processing Parameters on Rheology - Phase I Testing (U). WSRC-TR-2003-00364, Savannah River Site, Aiken, SC 29808 (2003). 
WSRC-TR-2004-00334

Revision 0

\section{APPENDIX A. TAYLOR VORTICES}


Curves showing the predicted location of Taylor vortices, or secondary flows, were included on many of the figures in the controlled shear rate flow curve discussion. The equation was given in generic form in section 2.2.4.1 equation [3]. The shear stress below which Taylor vortices are expected to form is given in terms of the rheometer bob and beaker radii and shear rate as a function of the slurry density by Smith (2002):

$$
\tau_{\text {Taylor vortices }}=\rho_{\text {slurry }} * \frac{\left(R_{\text {bea ker }}-R_{\text {bob }}\right)^{2.5}}{41.3 * \sqrt{R_{\text {bea } \mathrm{ker}}}} * \dot{\gamma}^{2}
$$

where $\rho_{\text {slurry }}$ is the slurry density and R symbolizes radius (inside for beaker and outside for bob). The interrelationship between this boundary and typical sludge, SRAT product, and SME product properties was investigated as part of the method development work. This in turn was used to constrain some of the rheology protocols developed from the method development work and presented in Koopman (2004b). Design bases rheological ranges, Table 13, were used to construct the expected ranges of slurry rheological properties for comparison with the curves of Taylor vortices.

Table 13. Rheological Bases for DWPF Slurries

\begin{tabular}{|l|c|c|c|c|}
\hline Slurry Type & $\begin{array}{c}\text { Lower Yield Stress, } \\
\text { dynes/cm }\end{array}$ & $\begin{array}{c}\text { Upper Yield Stress, } \\
\text { dynes/cm }\end{array}$ & $\begin{array}{c}\text { Lower } \\
\text { Consistency, cP }\end{array}$ & $\begin{array}{c}\text { Upper } \\
\text { Consistency, cP }\end{array}$ \\
\hline Sludge & 25 & 100 & 4 & 12 \\
\hline SRAT Product & 15 & 50 & 5 & 12 \\
\hline SME Product & 25 & 150 & 10 & 40 \\
\hline
\end{tabular}

Figure 54 presents the design operating region for sludge feed to DWPF. Simultaneously plotted on this figure are the predicted locations for the onset of Taylor vortices at low density $(1.08 \mathrm{~g} / \mathrm{ml})$ and high density $(1.22 \mathrm{~g} / \mathrm{ml})$ for both the MV1 and Z41 bobs with their respective annular gaps. A second set of Taylor vortices curves is given for the same densities and the MV2 and Z38 bobs with their respective annular gaps. 
WSRC-TR-2004-00334

Revision 0

Figure 54. Shear Rate Range Guidance for DWPF Sludge

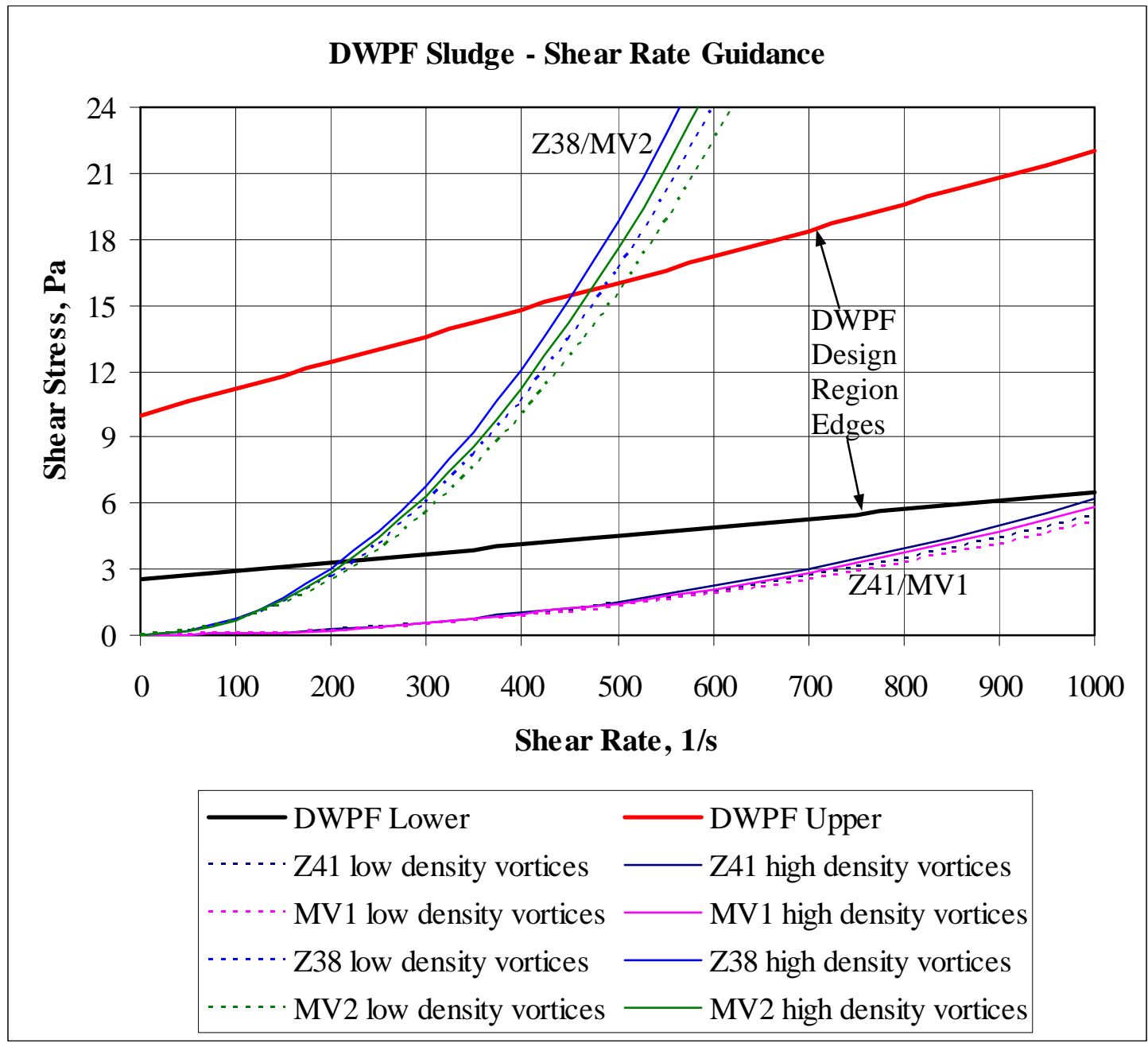

It is clear from Figure 54 that any DWPF sludge with rheological properties within the rheological box defined by the DWPF lower and upper limit curves can be measured out to a shear rate of 1000/s without encountering Taylor vortices when using MV1 and Z41 bobs. If, however, the MV2 or Z38 bobs must be used because of the presence of a chunky solid such as sand, coal or frit, then Taylor vortices could be encountered at shear rates ranging anywhere from 200/s to 500/s. Figure 55 gives a similar set of information for SRAT products. 
Figure 55. Shear Rate Range Guidance for DWPF SRAT Products

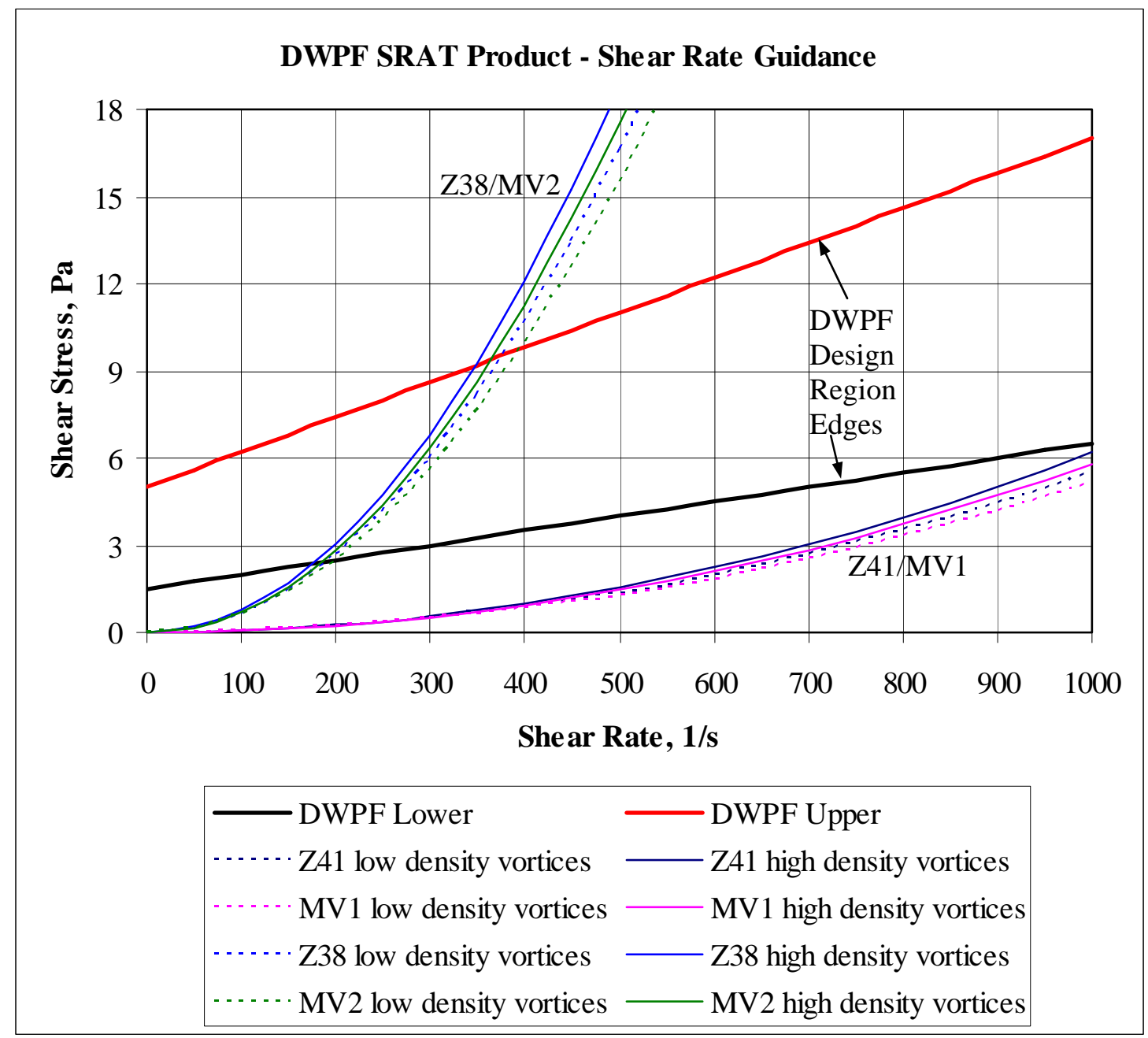

Similar conclusions can be drawn for SRAT products with densities from 1.08 to $1.22 \mathrm{~g} / \mathrm{mL}$ to those drawn for sludges. Figure 56 gives the corresponding information for SME products. 
Figure 56. Shear Rate Range Guidance for DWPF SME Products

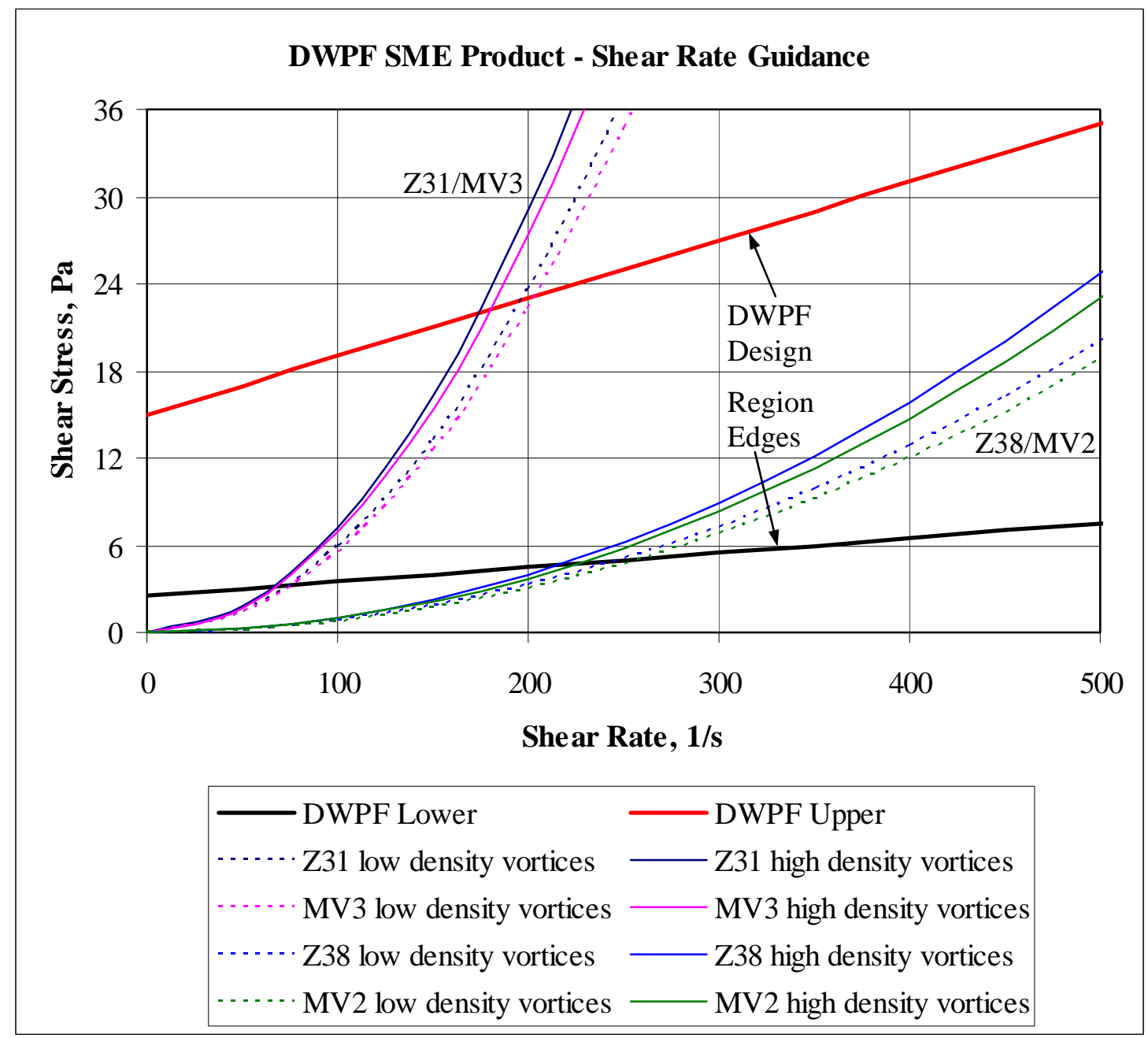

Figure 56 indicates that SME products are more susceptible to Taylor vortices than either SRAT products or sludges using the typical measurement geometries. The Z31 and MV3 geometry would typically be limited to maximum shear rates less than $80-150 /$ s for samples inside the DWPF design basis. Curves are given for densities of 1.3 and $1.6 \mathrm{~g} / \mathrm{mL}$. Samples are often encountered that are above the DWPF design basis. They should pose no problem for this approach. Samples below the DWPF design basis are likely to have other issues that are of more significance than Taylor vortices, such as limited frit suspension capability. 
WSRC-TR-2004-00334

Revision 0

End of the report. 


\section{Distribution:}

M. S. Miller, 704-S

J. E. Occhipinti, 704-S

R. M. Hoeppel, 704-27S

H. H. Elder, 703-H

J. F. Iaukea, 704-30S

J. W. Ray, 704-S

A. B. Sanders, 704-27S

W. B. Van-Pelt, 704-S

E. W. Holtzscheiter, SRNL

D. A. Crowley, 999-W

S. L. Marra, 999-W

T. B. Calloway, 999-W

N. E. Bibler, SRNL

C.M. Jantzen, SRNL

J. R. Harbour, 773-42A

G. C. Wicks, SRNL

R. E. Eibling, 999-W

M. E. Stone, 999-W

T. L. Fellinger, SRNL

E. K. Hansen, 999-W

T. M. Jones, 999-W

P. R. Burket, 773-42A 